\title{
Specific or non-specific exercises for patients with low back pain and movement control impairment
}

Citation for published version (APA):

Saner-Bissig, J. H. (2018). Specific or non-specific exercises for patients with low back pain and movement control impairment: What works? [Doctoral Thesis, Maastricht University]. Maastricht University. https://doi.org/10.26481/dis.20180906js

Document status and date:

Published: 01/01/2018

DOI:

10.26481/dis.20180906js

Document Version:

Publisher's PDF, also known as Version of record

\section{Please check the document version of this publication:}

- A submitted manuscript is the version of the article upon submission and before peer-review. There can be important differences between the submitted version and the official published version of record.

People interested in the research are advised to contact the author for the final version of the publication, or visit the DOI to the publisher's website.

- The final author version and the galley proof are versions of the publication after peer review.

- The final published version features the final layout of the paper including the volume, issue and page numbers.

Link to publication

\footnotetext{
General rights rights.

- You may freely distribute the URL identifying the publication in the public portal. please follow below link for the End User Agreement:

www.umlib.nl/taverne-license

Take down policy

If you believe that this document breaches copyright please contact us at:

repository@maastrichtuniversity.nl

providing details and we will investigate your claim.
}

Copyright and moral rights for the publications made accessible in the public portal are retained by the authors and/or other copyright owners and it is a condition of accessing publications that users recognise and abide by the legal requirements associated with these

- Users may download and print one copy of any publication from the public portal for the purpose of private study or research.

- You may not further distribute the material or use it for any profit-making activity or commercial gain

If the publication is distributed under the terms of Article $25 \mathrm{fa}$ of the Dutch Copyright Act, indicated by the "Taverne" license above, 


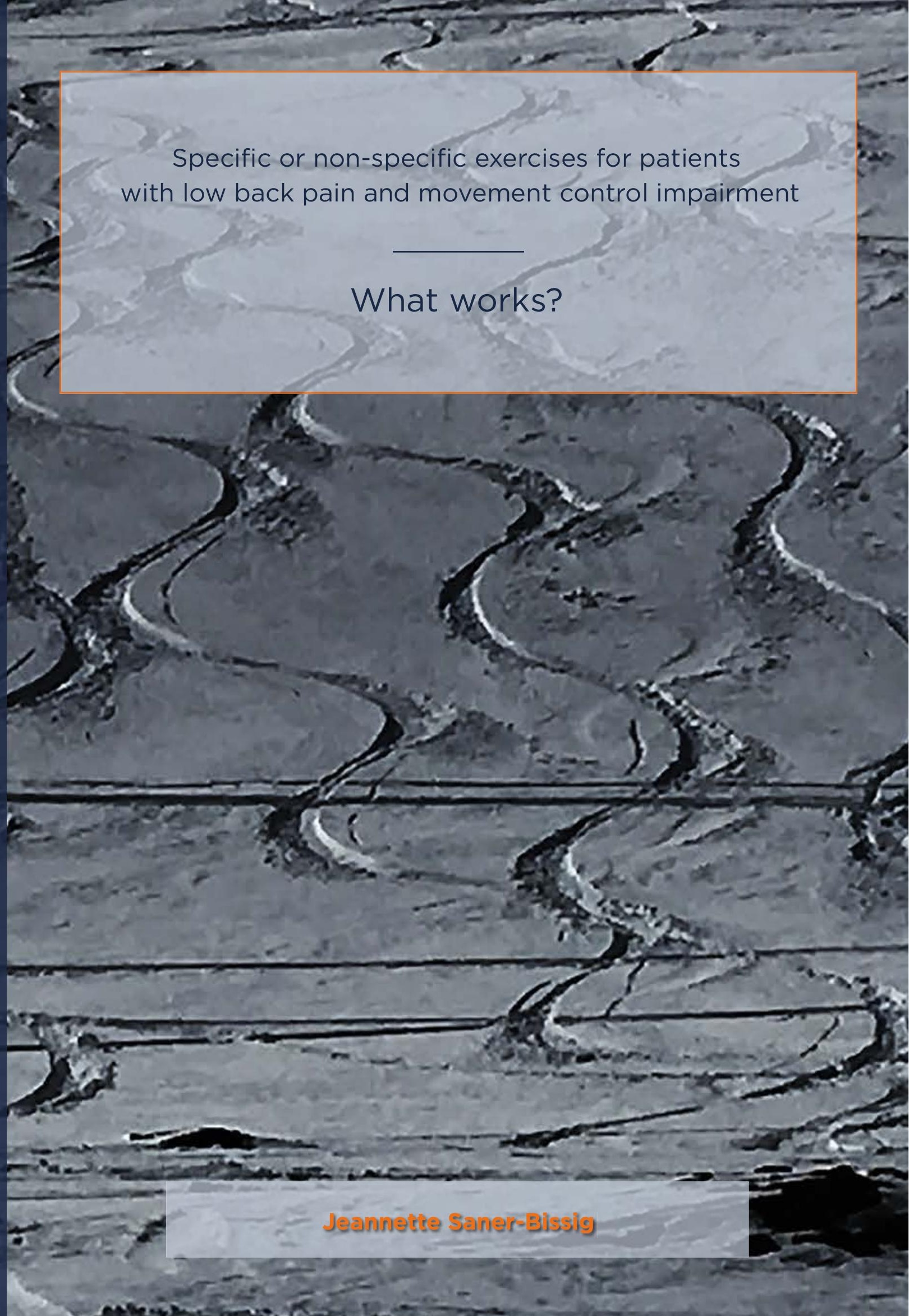





\title{
Specific or non-specific exercises for patients with low back pain and movement control impairment - What works?
}

\author{
DISSERTATION \\ to obtain the degree of Doctor at Maastricht University, \\ on the authority of the Rector Magnificus Prof.dr. Rianne M. Letschert \\ in accordance with the decision of the Board of Deans, \\ to be defended in public on
}

Thursday 6th September 2018 at 16.00 hours

by

Jeannette Saner-Bissig 


\section{Supervisor:}

Prof. dr. R. A. de Bie

\section{Co supervisors:}

Prof. dr. H. Luomajoki (ZHAW, Switzerland)

Dr. J. P. Kool (Kliniken Valens, Switzerland)

Dr. J. M. Sieben

\section{Assessment committee:}

Prof. dr. L. W. van Rhijn (Chair)

Prof. dr. R.H.H. Engelbert (Universiteit van Amsterdam)

Prof. dr. A.F. Lenssen

Dr. M. L. Verra (Inselspital Bern, Switzerland)

Dr. H. van Santbrink

ISBN: 9789493019805

Financial contribution:

Swiss National Science Foundation (SNSF) (Project No. 127240)

Zurich University of Applied Sciences, Winterthur, Switzerland 


\section{Contents}

Chapter 1 Introduction

Chapter 2 Study protocol: Movement control exercise versus general exercise to reduce disability in patients with low back pain and movement control impairment. A randomised controlled trial

Chapter 3 A tailored exercise program versus general exercise for a subgroup of patients with low back pain and movement control impairment:

Short-term results of a randomised controlled trial

Chapter 3A Supplement Exercise Procedures

Chapter 4 A tailored exercise program versus general exercise for a subgroup of patients with low back pain and movement control impairment:

a randomised controlled trial with one-year follow-up

Chapter 5 The patient-specific functional scale - its construct validity and validity to change in patients with shoulder and low back pain

Chapter 6 Patients' strategies and barriers to successful long-term adherence to home-based exercise programmes in physiotherapy

Chapter 7 General Discussion

Summary

Zusammenfassung

Valorisation

Acknowledgment

About the autor 

Chapter

Introduction 



\section{Background and my motivation to perform this research}

During my many years of clinical experience as a physiotherapist specialising in musculoskeletal disorders, approximately half of my clients have sought help for back-related problems. An acute phase of low back pain can be very painful, but is in most cases selflimiting. These observations are well supported by current literature $(1,2)$. My expertise in manual therapy and exercise therapy has allowed me in many cases to effectively manage this condition (3-5). Through my commitment as a faculty member at the McKenzie Institute (Mechanical Diagnosis and Therapy) $(6,7)$, my special interest in patient education and patient self-treatment was enhanced. However, in line with the results of a recent review, as well as the Swiss National Survey of 2014, I also observed recurring pain episodes and chronicity of pain and complaints in some patients $(2,8)$. The wish to provide my patients with a means to prevent these recurrent episodes was my motivation for the further development of my skills. But what treatment should we be recommending to patients whose problems had become chronic: what advice, which exercises and what type of behaviour? Is it enough to simply improve "general fitness"? Can we tackle the specific physical impairment of the individual patient? In general, how do we achieve long-term benefits for the patient?

All these questions were being addressed by a nationally-funded multi-centre trial at the Zurich University of Applied Sciences, in which I had the opportunity to participate. The aim of this project, namely to test a treatment that had already been in use for many years but which had not been studied under structured, controlled, experimental conditions, was absolutely in line with my professional experience (9).

From a personal viewpoint, it was about changing my perspective: from clinical experience to research - both quantitative and qualitative - and back again. My clinical experience was of considerable value in the management of this multi-centre project, since a large number of people were involved. Another of my objectives was to deliver evidencebased results for the benefit of my fellow physiotherapists. Evidence helps them to understand their clinical processes and provides indications for clinical decision making. Thus, ultimately, improves treatment and brings greater wellbeing to patients.

\section{Implications of low back pain for society and the individual patient}

The high prevalence rate of low back pain (LBP) and the associated large socio-economic burden on societies is well documented. The most recently published study on the global burden of this disease reviewed 117 articles and 5 surveys on acute and chronic low back pain (10). They concluded that LBP was ranked as the greatest contributor to global disability, measured in terms of Years lived with Disability (YLD). Globally, point prevalence for low back pain was shown to be $9.4 \%$ (95\% Cl 9.0 to 9.8). Age-standardised prevalence was highest in Western Europe, with 15\% of the population (between 0 and 100 years of 
age) suffering from low back pain. In terms of the overall burden, LBP ranked sixth when measured by Disability-Adjusted Life Years (DALY). DALY is calculated by combining Years of Life Lost (YLL) due to premature mortality and Years Lived with Disability (YLD). For LBP, YLD and DALY are equal, since no early mortality is assumed.

In Switzerland in 2012, a point prevalence of $40 \%$ for mild-to-severe low back pain was reported by adults living in private households (2). This rate is rising with increased age. The typical course of acute LBP is initially favourable, with a marked and spontaneous reduction in pain and disability being expected within the first six weeks. Despite this, in 2010, statistics revealed that 32,096 patients in Switzerland $(0.4 \%$ of total population) were hospitalised as a result of back pain (8). In patients suffering from LBP, a recurrence of symptoms has been recorded in the majority of cases (11). The strongest predictor of the occurrence of a new episode of LBP is a history of previous episodes of LBP (12). Where LBP had occurred at least once in the previous four years, the recurrence rate was $88.1 \%$ (12). In the previous 12 months, every one in four women (25\%) and every one in six men (16.9\%) had reported chronic back pain, defined as persistent pain lasting longer than three months (13).

Statistics for indirect, non-curative costs (e.g. work absence) in Switzerland are available only for all musculoskeletal disorders combined. But the latter accounted for costs of some 4.4 billion Swiss Francs in 2009. As the Ministry of Economics (Seco) reports, 26\% of all illness-related work absenteeism cases are due to musculoskeletal disorders (14). However, a survey of 1,000 Swiss inhabitants between the ages of 15 and 75 years reported work absenteeism of 1 to 6 days due to back pain in $18.6 \%$ of the participants. Some $6 \%$ missed work for a period of weeks and some $2.5 \%$ even for months (15).

These numbers are comparable across most European countries. In Germany, for example, $20.7 \%$ of participants in a National Survey (2009) reported chronic back pain, using the definition of three months or longer of daily back pain in the last year(13). Direct medical treatment costs of 3.6 billion Euros were related to non-specific low back pain (NSLBP) (13). Again, 75\% of total per patient low back pain costs are indirect costs resulting from work absenteeism or disablement (16). The total United Kingdom (UK) economic burden in 1998 from low back pain alone was calculated at around 18.6 billion Euros. Some $9 \%$ of patients in the UK complaining of back pain sought treatment from a physiotherapist. An estimated 150 million Euros annual costs for this physiotherapy were calculated (17).

In conclusion, a first episode of LBP is relatively "normal" in our society, with symptom reduction expected within a relatively short period of time. However, recurrences of episodes are common, with a small number of patients developing chronic non-specific low back pain. These patients account for the high costs resulting from this disorder, mainly due to work absenteeism and disablement. Therefore, physiotherapy treatment needs to be not only aimed at resolving the first episode, but also at addressing secondary or tertiary prevention of future LBP problems $(7,18)$. 


\section{Exercise therapy and non-specific low back pain}

The positive effects of exercise therapy in treating non-specific low back pain (NSLBP) have been well described $(4,19,20)$. NSLBP is defined as "low back pain that is not attributable to a recognisable, known specific pathology (e.g. infection, tumour, osteoporosis, fracture, structural deformity, inflammatory disorder (e.g. ankylosing spondylitis), radicular syndrome or cauda equina syndrome)" (1). In this large group with NSLBP, multifactorial aetiology (e.g. personal characteristics, psychosocial factors and workplace environment) is responsible for a great variability in clinical presentations. It can therefore be assumed that the NSLBP population can be further subdivided into various subgroups $(4,21)$. The latest guidelines point out the high evidence for the beneficial effects of exercises in patients with subacute and chronic $\operatorname{NSLBP}(22,23)$. Implementation of these recommendations may be difficult because of the wide variety of exercises that were tested $(22,23)$; however, most guidelines report that no one form of exercise is superior to another $(22,23)$. A meta-analysis showed a significant effect on chronic NSLBP of strength/resistance and coordination/stabilisation programs when compared to other interventions (21). The questions remain, however, as to which patients benefit from which exercises; which subgroup of NSLBP improves faster with which type of treatment; which factors influence the long-term benefit of the exercises?

\section{Subgroups and classifications of LBP and the introduction of movement control impairment}

One possible explanation for the lack of evidence for the effects of specific types of exercises may be the failure of research to adequately identify subgroups of patients with different presentations within the total group of NSLBP patients (24). Over the years, several classifications of NSLBP and specially targeted treatment programs have been developed. Subgroups have been classified based on different aspects, e.g. biomechanical assessment, psychosocial findings, neurophysiological or behavioural processes (25-27). In NSLBP with mainly biomechanical aspects, classifications are based on the assessment of movement. Firstly, major movement-oriented classifications can be drawn on the basis of the assessment of range of movement and movement repetitions in relation to pain as, for example, in Mechanical Diagnosis and Therapy (MDT) (28) or in Pathoanatomic Based Classification (PBC) (29). Secondly, classification can be based on the results of the assessment of the quality and alignment of movements and their relationship to pain, as in Movement System Impairment (MSI) (30) or the O'Sullivan Classification System (OCS) (31). The different classification systems became increasingly complex, were difficult to distinguish from one another and showed some overlap (26).

A new classification of Movement Control Impairment ( $\mathrm{MCl}$ ) resulted from the studies carried out by Luomajoki (32-35). Movement tests, formerly described in MSI and OSC 
classifications, were assessed for intrarater and interrater reliability. Tests showing the best results were grouped and formed the "movement control test battery". A further study discovered that two or more positively assessed movements can be discriminative for patients with low back pain. The $\mathrm{MCl}$ classification test battery can be easily applied in clinical practice since it does not need additional technical equipment; it is reasonably short and can be reliably performed without excessive training or experience. A controlled case series study $(n=38)$ was performed to test the possible relationship between subgroup and outcomes. Firstly, the $\mathrm{MCl}$ subgroup was identified using the test battery; secondly, movements were sub-classified into direction-specific impairments, as described in OSC. The subsequent exercise treatment had the aim of improving the movement control deficit with practice-based and home-based exercises. Results showed a significant post-treatment effect using the $\mathrm{MCl}$ tests, disability (Roland-Morris Disability Questionnaire) and function (Patient Specific Functional Scale).

These studies formed the basis of the present thesis. It was hypothesized that patients with $\mathrm{MCl}$ and a further sub-classification can be effectively treated using specific exercises. Although the studies did not show the causality of $\mathrm{MCl}$ on NSLBP, the close relationship of the outcomes described above were further supported by pathoanatomical and biomechanical explanations. Firstly, subacute and chronic LBP maladaptive processes, such as avoidance or overuse, may lead to potentially harmful postures and movements (36-38). These 'faulty' unaligned movements damage tissues and may cause pain $(37,39,40)$. Further, an additional rationale originates from the theory of "Relative Flexibility" which suggests that movement is always lead by the pathway of least resistance, i.e. a stiff hip will lead to potential overload of the lumbar spine and vice versa (30). This overload may also lead to damaged tissue and pain in the lumbar spine. Thus, a specific exercise program for this $\mathrm{MCl}$ subgroup, addressing the dynamic and static control of movements in the lumbar spine, the pelvic area and the hip, was a promising treatment option and of interest to research.

\section{Historical perspective to movement control exercise treatments}

Different concepts have been used over the past decades to improve posture and/or movement alignment. Early physiotherapy approached postural problems through very strict stabilising exercises, using isometric muscle training and general advice to keep a "straight back" (41). In the early 1990s, the "segmental stabilisation" concept, one of the first in physiotherapy to be based on the results of research, treated the signs and symptoms of the patient. It addressed a specific finding, i.e. the delayed activation of the local stabilising muscles of the spine, $m$. transversus abdominis or mm. multifidi $(42,43)$. The developed treatment strategy is known as Motor Control Exercise (MCE) and incorporates a voluntary contraction of the deep abdominal muscles during exercise. The described exercises progress from low load static performance to individually adjusted high 
load demands. Many other therapeutic programs have included the so-called "abdominal hollowing" concept - activation of transversus abdominis muscels - in exercise programs for NSLBP. About 15 years ago, Sahrmann presented her comprehensive work named "Movement Systems Impairment" (MSI) (44). The test and treatment procedures addressed movement systems with suboptimal sequences when changing positions. The treatment approach is based on individual assessment and changing movement sequences which are relevant to the patient's symptoms. 0'Sullivan presented his directionspecific O'Sullivan Classification system (OSC) in 2005 (31). This classification is named after the painful movement direction arising from a control deficit of the symptom-provoking spinal segment. His treatment approach combines the learning process of performing non-provocative movements with a cognitive psychosocial approach, and is known as Cognitive Functional Treatment (CFT).

All these treatment approaches have been studied during the past eight years, since the start of our projects. Two Cochrane reviews, published in 2016, summarise the effects of Motor Control Exercises (MCE). They report no positive benefits of MCE over other forms of exercise in the acute and subacute phases of NSLBP (45), as well as for patients with chronic NSLBP $(46,47)$. As for the treatment in Movement System Impairment (MSI), no differences were reported when compared to a non-specific exercise program in the chronic pain population (48). Furthermore, a randomised controlled trial reported superior (but not significant) effects of the Cognitive Functional Treatment (CFT) over combined manual therapy/exercise treatment $(49,50)$. However, the authors emphasise the importance of the psychosocial approach, including the therapeutic alliance between physiotherapist and patient. Additionally, the CFT approach includes patient-specific behavioural aspects in information, exercise dosage and functions in daily life. This cognitive aspect was not part of our treatment procedure.

The studies presented in this thesis are closely related and based on these three concepts. The treatment of Movement Control Impairment (MCl) is based on the OSC classification and the treatment progressions are influenced by MSI and MCE. However, at the start of our project, the treatment had not been tested under controlled conditions. The question remains as to whether patients in the $\mathrm{MCl}$ subgroup can benefit from a specific treatment procedure. Is this newly determined subgroup the first that can be efficiently treated with specifically-tailored exercises? In general, it remains to be seen whether the concept of an impaired movement control can be responsible for NSLBP.

\section{Objectives of this PhD thesis}

The objectives of this thesis are:

To analyse the effects of specifically-tailored exercises on function and activity in patients with NSLBP, who have been assessed and classified into the subgroup with movement control impairment (Chapters 2, 3, 4,) 
- To evaluate the quantitative and qualitative long-term effects of this specific exercise treatment when compared with general exercise treatment (Chapters 4, 6)

- To explain the results, influences and mechanisms of the trial from the critical theoretical and patient-perceived perspectives (Chapters 5, 6)

- To generate knowledge for evidence-based practice recommendations in the treatment of a specific subgroup of NSLBP patients with $\mathrm{MCl}$ (all chapters)

The following outline summarises the aims and perspectives of the chapters comprising this thesis.

\section{Chapter 2-Protocol}

The research protocol of the randomised, controlled trial (RCT) builds on previous reports concerning the stages of NSLBP, subgroups in NSLBP, effects of exercise therapy and treatment modalities. The subgroup of movement control impairment $(\mathrm{MCl})$ is defined, together with its inclusion/exclusion criteria. This chapter further describes the methodological procedures of the RCT, in which a tailored exercise program is compared to general exercise for a subgroup of patients with low back pain and movement control impairment.

Chapter 3 - Short-term results and Supplement

This chapter reports the short-term results of the RCT. In the supplement, it also includes a detailed description and illustration of the two treatments.

Chapter 4- Long-term results

This chapter reports the long-term results by group comparison in the RCT. The main outcome measures are disability and activity.

Chapter 5 - Clinimetrics of the Patient Specific Functional Scale

This chapter reports on the clinimetric properties of the primary outcome used in the RCT, namely the Patient Specific Functional Scale (PSFS). Results of two trials (shoulder pain, low back pain) were merged to evaluate the validity and responsiveness of this measurement instrument for both research and clinical purposes.

Chapter 6 - Patients' perceptions of adherence - qualitative evaluation

This chapter identifies themes which are of importance to the patient in the long-term exercise process. Of particular interest to us was the analysis of the topic "adherence to exercise" and the influencing factors.

Chapter 7 - General discussion

In this chapter, the main results of the thesis are discussed against the background of recent studies and from a research methodological perspective. It includes a critical appraisal of the results with regard to the objectives of this thesis. 
Outline of the thesis

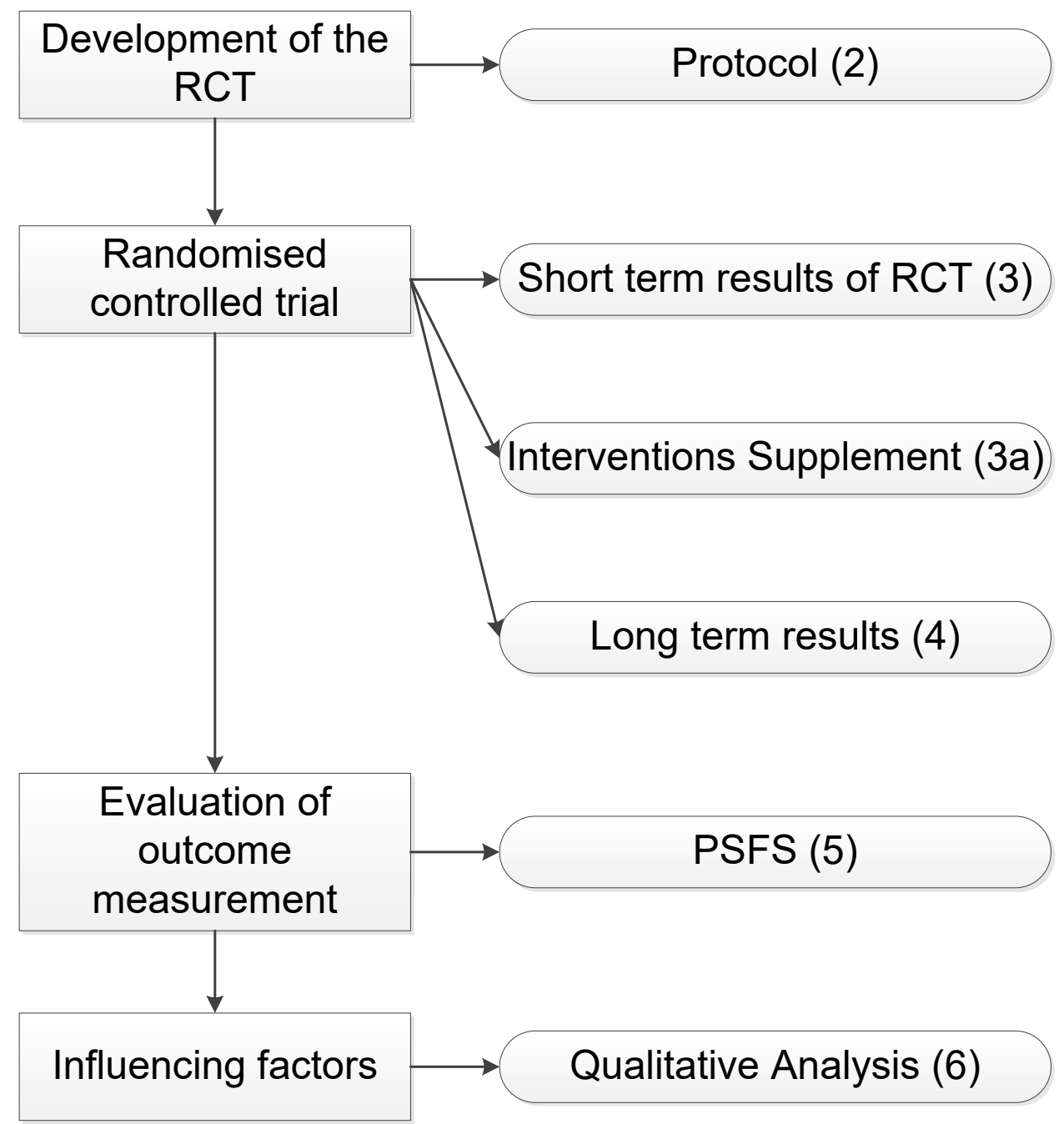




\section{References}

1. Airaksinen O, Brox J, Cedraschi C, Hildebrandt J, Klaber-Moffett J, Kovacs F, et al. Chapter 4. European guidelines for the management of chronic nonspecific low back pain. Eur Spine J. 2006;15 Suppl 2:S192300. PubMed PMID: 16550448.

2. da CMCL, Maher CG, Hancock MJ, McAuley JH, Herbert RD, Costa LO. The prognosis of acute and persistent low-back pain: a meta-analysis. CMAJ. 2012;184(11):E613-24. doi: 10.1503/cmaj.111271. PubMed PMID: 22586331; PubMed Central PMCID: PMC3414626.

3. Apeldoorn AT, van Helvoirt $H$, Meihuizen $H$, Tempelman $H$, Vandeput $D, K$ nol DL, et al. The Influence of Centralization and Directional Preference on Spinal Control in Patients With Nonspecific Low Back Pain. J Orthop Sports Phys Ther. 2016;46(4):258-69. doi: 10.2519/jospt.2016.6158. PubMed PMID: 26813757.

4. Hayden JA, van Tulder MW, Malmivaara A, Koes BW. Exercise therapy for treatment of non-specific low back pain. Cochrane Database Syst Rev. 2005(3):CD000335. Epub 2005/07/22. doi: 10.1002/14651 858.CD000335.pub2. PubMed PMID: 16034851

5. Koes BW, van Tulder MW, Thomas S. Diagnosis and treatment of low back pain. BMJ. 2006;332 (7555):1430-4. Epub 2006/06/17. doi: 332/7555/1430 [pii] 10.1136/bmj.332.7555.1430. PubMed PMID: 16777886; PubMed Central PMCID: PMC1479671.

6. McKenzie R, May S. The lumbar spine : mechanical diagnosis and therapy. 2nd ed., reprinted 2004 ed: Waikanae, NZ : Spinal Publications; 2004. 2 Bde. (374 S.) p.

7. Saner-Bissig J, Clare H. McKenzie - Mechanische Diagnose und Therapie: Stuttgart : Thieme; 2007. 91 S. p.

8. Bundesamt für Statistik (BFS); Tania Andreani SB, Pascale Gazareth, Dimitri Hauri, Martine Kaeser, Renaud Lieberherr,, Michael Lindner J-FM, Marco Storni, Giovanni Teotino, Erwin Wüest. Gesundheitsstatistik 2014. Bundesamt für Statistik, CH-2010 Neuchâtel; 2014.

9. Saner J, Kool J, de Bie RA, Sieben JM, Luomajoki H. Movement control exercise versus general exercise to reduce disability in patients with low back pain and movement control impairment. A randomised controlled trial. BMC Musculoskelet Disord. 2011;12:207. doi: 10.1186/1471-2474-12-207. PubMed PMID: 21943318; PubMed Central PMCID: PMC3189916.

10. Hoy D, March L, Brooks P, Blyth F, Woolf A, Bain C, et al. The global burden of low back pain: estimates from the Global Burden of Disease 2010 study. Ann Rheum Dis. 2014;73(6):968-74. doi: 10.1136/annrheumdis-2013-204428. PubMed PMID: 24665116.

11. Lemeunier N, Leboeuf-Yde C, Gagey $O$. The natural course of low back pain: a systematic critical literature review. Chiropractic \& Manual Therapies. 2012;20(1):33. doi: 10.1186/2045-709x-20-33.

12. Kolb E, Canjuga M, Bauer GF, Läubli T. Course of back pain across 5 years. Spine. 2011;36.

13. Robert Koch-Institut (Hrsg) Gemeinsam getragen von RKI und Destatis. RKI B. Gesundheit in Deutschland. Gesundheitsberichterstattung des Bundes. In: Robert Koch-Institut (Hrsg); Gemeinsam getragen von RKI und Destatis. RKI B, editor. 2015.

14. Staatssekretariat für Wirtschaft SECO; Ralph Krieger MGuMV. Arbeit und Gesundheit 2012. 2015.

15. Gerfin A. Rückenreport Schweiz 2011; Die Erhebung zur Rückengesundheit von Herrn und Frau Schweizer. In: Schweiz R, editor. 2011.

16. Juniper M, Le TK, Mladsi D. The epidemiology, economic burden, and pharmacological treatment of chronic low back pain in France, Germany, Italy, Spain and the UK: a literature-based review. Expert opinion on pharmacotherapy. 2009;10(16):2581-92. doi: 10.1517/14656560903304063. PubMed PMID: 19874246.

17. Maniadakis N, Gray A. The economic burden of back pain in the UK. Pain. 2000;84(1):95-103. PubMed PMID: 10601677.

18. Macedo LG, Bostick GP, Maher CG. Exercise for prevention of recurrences of nonspecific low back pain. Phys Ther. 2013;93(12):1587-91. doi: 10.2522/ptj.20120464. PubMed PMID: 23813085.

19. Airaksinen O, Brox J, Cedraschi C, Hildebrandt J, Klaber-Moffett J, Kovacs F, et al. Chapter 5. European guidelines for the management of chronic nonspecific low back pain. Eur Spine J. 2006;15 Suppl 2:S192300. PubMed PMID: 16550448. 
20. Choi B, K. L., Verbeek Jos H, Tam Wilson W-S, Jiang Johnny Y. Exercises for prevention of recurrences of low-back pain. Cochrane Database of Systematic Reviews [Internet]. 2010; (1). Available from: http://www.mrw.interscience.wiley.com/cochrane/clsysrev/articles/CD006555/frame.html.

21. Searle A, Spink M, Ho A, Chuter V. Exercise interventions for the treatment of chronic low back pain: a systematic review and meta-analysis of randomised controlled trials. Clinical rehabilitation. 2015;29(12):1155-67. doi: 10.1177/0269215515570379. PubMed PMID: 25681408.

22. Koes BW, van Tulder M, Lin CW, Macedo LG, McAuley J, Maher C. An updated overview of clinical guidelines for the management of non-specific low back pain in primary care. Eur Spine J. 2010;19(12):2075-94. doi: 10.1007/s00586-010-1502-y. PubMed PMID: 20602122; PubMed Central PMCID: PMC2997201.

23. Bundesärztekammer (BÄK) KBK, Arbeitsgemeinschaft der Wissenschaftli-chen Medizinischen Fachgesellschaften (AWMF). Nationale VersorgungsLeitlinie Nicht-spezifischer Kreuz-schmerz - Langfassung, 2017. Available from: www.kreuzschmerz.versorgungsleitlinien.de.

24. Wand BM, O'Connell NE. Chronic non-specific low back pain - sub-groups or a single mechanism? BMC Musculoskelet Disord. 2008;9:11. doi: 10.1186/1471-2474-9-11. PubMed PMID: 18221521; PubMed Central PMCID: PMC2266926.

25. Karayannis NV, Jull GA, Hodges PW. Physiotherapy movement based classification approaches to low back pain: comparison of subgroups through review and developer/expert survey. BMC Musculoskelet Disord. 2012;13:24. doi: 10.1186/1471-2474-13-24. PubMed PMID: 22348236; PubMed Central PMCID: PMC3395852.

26. Karayannis NV, Jull GA, Hodges PW. Movement-based subgrouping in low back pain: synergy and divergence in approaches. Physiotherapy. 2016;102(2):159-69. doi: 10.1016/j.physio.2015.04.005. PubMed PMID: 26126426.

27. Apeldoorn AT, Bosselaar H, Ostelo RW, Blom-Luberti T, van der Ploeg T, Fritz JM, et al. Identification of patients with chronic low back pain who might benefit from additional psychological assessment. Clin J Pain. 2012;28(1):23-31. doi: 10.1097/AJP.0b013e31822019d0. PubMed PMID: 21677570.

28. McKenzie RM, S. The Lumbar Spine: Mechanical Diagnosis \& Therapy. 2nd Edition ed: Spinal Publications New Zealand Ltd; 2003.

29. Petersen T, Olsen S, Laslett M, Thorsen H, Manniche C, Ekdahl C, et al. Inter-tester reliability of a new diagnostic classification system for patients with non-specific low back pain. Aust J Physiother. 2004;50(2):85-94. PubMed PMID: 15151492

30. Sahrmann SA. Diagnosis and treatment of movement impairment syndromes: St. Louis: Mosby, Inc; 2002.

31. O'Sullivan P. Diagnosis and classification of chronic low back pain disorders: Maladaptive movement and motor control impairments as underlying mechanism. Manual Therapy. 2005;10(4):242-55. PubMed PMID: 69.

32. Luomajoki H. Movement Control Impairment as a subgroup of non-specific low back pain. Institute of Clinical Medicine, School of Medicine, Faculty of Health Sciences. 22.10.2010 ed. Kuopio: Eastern Finland University Library/Sales of Publications; 2010. p. 70.

33. Luomajoki $\mathrm{H}, \mathrm{Kool} \mathrm{J}$, de Bruin ED, Airaksinen O. Reliability of movement control tests in the lumbar spine. BMC Musculoskelet Disord. 2007;8:90. Epub 2007/09/14. doi: 1471-2474-8-90 [pii] 10.1186/1471-24748-90. PubMed PMID: 17850669; PubMed Central PMCID: PMC2164955.

34. Luomajoki H, Kool J, de Bruin ED, Airaksinen O. Movement control tests of the low back; evaluation of the difference between patients with low back pain and healthy controls. BMC Musculoskelet Disord. 2008;9:170. Epub 2008/12/26. doi: 1471-2474-9-170 [pii] 10.1186/1471-2474-9-170. PubMed PMID: 19108735; PubMed Central PMCID: PMC2635372.

35. Luomajoki H, Kool J, de Bruin ED, Airaksinen O. Improvement in low back movement control, decreased pain and disability, resulting from specific exercise intervention. Sports Med Arthrosc Rehabil Ther Technol. 2010;2:11. Epub 2010/04/27. doi: 1758-2555-2-11 [pii] 10.1186/1758-2555-2-11. PubMed PMID: $20416091 ;$ PubMed Central PMCID: PMC2873574.

36. Moseley GL. I can't find it! Distorted body image and tactile dysfunction in patients with chronic back pain. Pain. 2008;140(1):239-43. Epub 2008/09/13. doi: S0304-3959(08)00444-2 [pii] 10.1016/j.pain. 2008.08.001. PubMed PMID: 18786763. 
37. van Dieen JHM, L; Hodges, P.W. Motor control changes and low back pain: cause or effect? In: Hodges P, editor. Spinal control : the rehabilitation of back pain : state of the art and science: Edinburgh : Churchill Livingstone Elsevier; 2013. p. 207.

38. Moseley GL. Trunk muscle control and back pain: chicken, egg, neither or both? In: Hodges P, editor. Spinal control : the rehabilitation of back pain : state of the art and science: Edinburgh : Churchill Livingstone Elsevier; 2013. p. $123 \mathrm{ff}$.

39. Van Dillen LR, Sahrmann SA, Norton BJ, Caldwell CA, McDonnell MK, Bloom NJ. Movement system impairment-based categories for low back pain: stage 1 validation. J Orthop Sports Phys Ther. 2003;33(3):12642. doi: 10.2519/jospt.2003.33.3.126. PubMed PMID: 12683688.

40. Hodges PW. Spinal control : the rehabilitation of back pain : state of the art and science: Edinburgh : Churchill Livingstone Elsevier; 2013. 327 S. p.

41. Kaganas G. Physiotherapie bei degenerativ-rheumatischen Erkrankungen der Wirbelsäule: Basel : Geigy S.A.; 1967. 94 S. p.

42. Richardson C, Hodges PW, Hides J. Therapeutic exercise for lumbo-pelvic stabilisation : a motor control approach for the treatment and prevention of low back pain. 2nd ed. ed: Edinburgh : Churchill Livingstone : [Online:] Elsevier ScienceDirect Books; 2004. Online-Ressource p.

43. Richardson C, Hodges $P$, Hides J. Segmentale Stabilisation im LWS- und Beckenbereich : therapeutische Übungen zur Behandlung von Low Back Pain: München : Elsevier, Urban und Fischer; 2009. 266 S. p.

44. Sahrmann S. Diagnosis and treatment of movement impairment syndromes: St. Louis, Mo. : Mosby; 2002. 460 S. p.

45. Macedo LG, Saragiotto BT, Yamato TP, Costa LO, Menezes Costa LC, Ostelo RW, et al. Motor control exercise for acute non-specific low back pain. Cochrane Database Syst Rev. 2016;2:CD012085. doi: 10.1002/14651858.CD012085. PubMed PMID: 26863390.

46. Saragiotto BT, Maher CG, Yamato TP, Costa LO, Costa LC, Ostelo RW, et al. Motor Control Exercise for Nonspecific Low Back Pain: A Cochrane Review. Spine (Phila Pa 1976). 2016;41(16):1284-95. doi: 10.1097/BRS.0000000000001645. PubMed PMID: 27128390.

47. Saragiotto BT, Maher CG, Yamato TP, Costa LO, Menezes Costa LC, Ostelo RW, et al. Motor control exercise for chronic non-specific low-back pain. Cochrane Database Syst Rev. 2016(1):CD012004. doi: 10.1002/14651858.CD012004. PubMed PMID: 26742533.

48. Van Dillen LR, Norton BJ, Sahrmann SA, Evanoff BA, Harris-Hayes M, Holtzman GW, et al. Efficacy of classification-specific treatment and adherence on outcomes in people with chronic low back pain. A one-year follow-up, prospective, randomized, controlled clinical trial. Man Ther. 2016;24:52-64. doi: 10.1016/j.math.2016.04.003. PubMed PMID: 27317505; PubMed Central PMCID: PMC4921225.

49. Vibe Fersum K, O'Sullivan P, Skouen JS, Smith A, Kvale A. Efficacy of classification-based cognitive functional therapy in patients with non-specific chronic low back pain: a randomized controlled trial. Eur J Pain. 2013;17(6):916-28. doi: 10.1002/j.1532-2149.2012.00252.x. PubMed PMID: 23208945.

50. O'Sullivan P, Caneiro JP, O'Keeffe M, O'Sullivan K. Unraveling the Complexity of Low Back Pain. J Orthop Sports Phys Ther. 2016;46(11):932-7. doi: 10.2519/jospt.2016.0609. PubMed PMID: 27802794. 


\section{Chapter}

Study protocol:

Movement control exercise versus general exercise to reduce disability in patients with low back pain and movement control impairment.

A randomised controlled trial 


\section{Abstract}

Background: Non-specific low back pain (NSLBP) in subacute and chronic stages can be treated effectively with exercise therapy. Research guidelines recommend evaluating different treatments in defined subgroups of patients with NSLBP. A subgroup of patients with movement control impairment ( $\mathrm{MCl}$ ) improved significantly on patient specific function and disability in a previous case series after movement control exercises. Current Controlled Trials ISRCTN80064281.

Methods/Design: In a randomised controlled trial (RCT) we will compare the effectiveness of movement control and general exercise in patients with $\mathrm{MCl}$. 106 participants aged 18 - 75 will be recruited in 5 outpatient hospital departments and 7 private practices. Patients randomly assigned to the movement control exercise group will be instructed to perform exercises according to their $\mathrm{MCl}$. The general exercise group will follow an exercise protocol aimed at improving endurance and flexibility. Patients in both groups will receive 9-18 treatments and will be instructed to do additional exercises at home.

The primary outcome is the level of disability assessed using the patient specific functional scale (PSFS) which links the perceived pain to functional situations and is measured before treatment and at 6 and 12 months follow-up. Secondary outcomes concern low back pain related disability (Roland Morris questionnaire, RMQ), graded chronic pain scale (GCPS), range of motion and tactile acuity.

Discussion: To our knowledge this study will be the first to compare two exercise programs for a specific subgroup of patients with NSLBP and $\mathrm{MCl}$. Results of this study will provide insight into the effectiveness of movement control exercise and contribute to our understanding of the mechanisms behind $\mathrm{MCl}$ and its relation to NSLBP.

Trial registration: Current Controlled Trials ISRCTN80064281 


\section{Background}

Low Back Pain (LBP) is one of the major concerns of current health care (1-5). Only $10 \%$ of LBP cases can be attributed to specific disorders like nerve root compression, vertebral fracture, tumour, infection, inflammatory diseases, spondylolisthesis or spinal stenosis. Consequently, NSLBP, in which the cause of symptoms is unknown, is diagnosed in about $90 \%$ of all patients and is a health problem of high economic importance (6).

Patients with NSLBP present diverse clinical findings, courses of disease and prognoses. They therefore make up a heterogeneous group of patients, which may explain why treatment effects in numerous studies looking at specific physiotherapy treatments in the NSLBP group are often discouraging. Identifying defined subgroups of patients within the NSLBP population has been a major focus in recent research (7-12). Current European guidelines encourage outcome studies in subgroups of patients with a shared diagnostic pattern or prognosis that might benefit from specific treatments (1). This research agenda is expected to reveal further evidence for the effect of treatments designed for specific subgroups (13). Of 767 RCTs about the effect of conservative treatment on chronic LBP performed and published between 1982 and 2008, 68 publications examined manual or exercise therapy, of which five studies had an additional subclassification and matched treatments (14).

There is no evidence for the effectiveness of exercise in patients with acute LBP of a less than 6 week duration $(1,15-17)$, but exercise therapy is effective in chronic and subacute LBP (16). There is evidence that home exercise may be effective in decreasing pain and disabiltiy, but results have shown no significant difference between exercise types on work disability $(1,18)$. There is moderate evidence suggesting that exercise therapy may prevent recurrences of LBP, but there is no evidence for a difference in effect between types of exercise $(19,20)$. Individually designed exercise programs are recommended (17) but the question remains as to which types of exercise are effective for which subgroups of patients.

Within the framework of a new NSLBP classification system developed by O'Sullivan, one of the subgroups of patients that can be distinguished contains those suffering from $\mathrm{MCl}$ (11). In a first step, this classification of NSLBP distinguishes between patients with non-mechanical disorders, and patients with mechanical disorders. Whereas in patients with non-mechanical NSLBP, psychosocial factors, fear and catastrophising play central roles, pain in relation to posture and movement is predominant in patients with mechanical NSLBP. Patients with mechanical NSLBP are further divided into those with movement impairment (MI) and movement control impairment (MCl). Patients with $\mathrm{MI}$ may suffer movement restrictions in single or multiple directions. $\mathrm{MCl}$ is defined as a deficit in the control of movements during functional daily activities. The range of the movement is not restricted in the $\mathrm{MCl}$ group.

Clinical tests to identify $\mathrm{MCl}$ were developed in recent research $(11,21-27)$. Further evaluation revealed six tests which reliably detect $\mathrm{MCl}$ in patients (28). (Add. files 1) These 
tests will be used to select patients with $\mathrm{MCl}$ for this study. Movement control tests are easy to perform in clinical practice. Tests and clinical presentation allow a further classification of movement control dysfunctions according to direction in extension, flexion, frontal plane and multi-directional MCls. (Table1)

Table 1: Five distinct directional patterns of movement control impairment (29) (personal communication)

\begin{tabular}{|c|c|c|c|}
\hline $\begin{array}{l}\text { Direction of movement } \\
\text { control impairment }\end{array}$ & Pain aggravation & Pain relief & Movement control deficit \\
\hline Flexion & $\begin{array}{l}\text { Sustained flexion of } \\
\text { lumbar spine, e.g. when } \\
\text { sitting }\end{array}$ & $\begin{array}{l}\text { Extension of lumbar spine, } \\
\text { e.g. when standing and } \\
\text { walking }\end{array}$ & $\begin{array}{l}\text { Difficulty controlling } \\
\text { lordosis in sitting and } \\
\text { flexed positions }\end{array}$ \\
\hline Active Extension & $\begin{array}{l}\text { Sustained extension of } \\
\text { lumbar spine }\end{array}$ & $\begin{array}{l}\text { Flexion of lumbar spine, } \\
\text { relaxing in flexed posture. } \\
\text { Breathing exercises }\end{array}$ & $\begin{array}{l}\text { Difficulty flexing when } \\
\text { sitting or breathing with } \\
\text { diaphragm }\end{array}$ \\
\hline Passive extension & $\begin{array}{l}\text { Extension of lumbar spine, } \\
\text { e.g. when standing or } \\
\text { walking slowly }\end{array}$ & $\begin{array}{l}\text { Flexion of lumbar spine, } \\
\text { e.g. while sitting }\end{array}$ & Tilting pelvis posteriorly \\
\hline Frontal pain control & $\begin{array}{l}\text { unilateral pain in unilateral } \\
\text { loading and sidebending }\end{array}$ & $\begin{array}{l}\text { Control pelvis and thorax } \\
\text { in frontal plain }\end{array}$ & $\begin{array}{l}\text { Maintain symmetric } \\
\text { posture }\end{array}$ \\
\hline Multidirectional pattern & Multidirectional & $\begin{array}{l}\text { Changing lumbar spine } \\
\text { position }\end{array}$ & $\begin{array}{l}\text { Difficulty assuming neutral } \\
\text { lordotic spinal positions }\end{array}$ \\
\hline
\end{tabular}

Specification of main symptoms and signs to classify the direction of movement control impairment

Two mechanisms are proposed to explain the impaired movement control behaviour. One relates to conditioning and habituation, which are important factors in motor learning. Patients use postures and movements that are potentially harmful due to maladaptive processes, like avoidance or overuse during the acute pain phase(30). Another mechanism is non-awareness of the posture's pain provocation. Altered cortical representation of the lumbar spine in the presence of pain may play an important role (31). Two point discrimination is decreased in patients with NSLBP, indicating changes in cortical representation because of NSLBP (32). Both mechanisms can either be induced by pain or be the cause of pain.

It is hypothesised that, once movement control is impaired, it results in repetitive mechanical deformation of innervated tissue and leads to increased nociceptive input to the central nervous system and, therefore, pain (33). All joint capsules, ligaments, tendons and muscles are possible pain sources, especially due to continuous strain or longstanding repetitive movements $(33,34)$. Repeated misuse of these tissues can also initiate the inflammatory cascade, a further cause of pain (35).

The inter-rater reliability of the clinical classification of NSLBP in $\mathrm{MI}$ and $\mathrm{MCl}$ is very high; in experts and raters with less experience, $k=0.85$ and $k=0.6$ respectively $(36,37)$. The inter-and intra-rater reliability of the previously described 6 active tests performed by the patient was evaluated as good to substantial (28). Validity of $\mathrm{MCl}$ tests is supported by significantly different results in healthy subjects, with 0.75 positive $\mathrm{MCl}$ tests $(95 \% \mathrm{Cl}$ 
0.55-0.95) and low back pain patients with 2.21 positive $\mathrm{MCl}$ tests $(95 \% \mathrm{Cl} 1.94-2.48$, effect size $d=1.18$ ) (38).

In a preceding prior controlled case series, 38 preselected patients with positive $\mathrm{MCl}$ tests were treated with an individualised movement control exercise program and showed improvements in $\mathrm{MCl}$ test performance associated with improvements in patient specific functional complaints and disability (39). The present study will compare movement control training with general exercise in a randomised controlled trial.

$\mathrm{MCl}$ exercises are often and falsely referred to as motor control exercises, spinal stabilisation or core stability exercises. While $\mathrm{MCl}$ exercises aim to improve function through repetitive normal use, the latter retrain delayed muscle activity first in order to improve control of the spine. Several trials and systematic reviews have evaluated the effect of stabilising exercises with conflicting results (40-44). A recent randomised placebo-controlled trial with a specific stabilisation program in a population with chronic low back pain found a significant improvement in activity in both the short and long term when measured with a patient specific functional scale (PSFS), and a short term improvement for global impression of recovery and disability (45). However, they did not subgroup the patients and the effects were not beyond the smallest minimal meaningful clinical change.

The general exercise treatment is based on treatments used in previous studies. Exercises were developed to improve endurance, strength and flexibility of the spinal tissues (14). Experimental studies have shown that these exercises involve many global and local muscles and improve the stability of the spine $(46,47)$. In an RCT of patients with subacute and chronic NSLBP, a course of only general exercise was compared with a combination of general exercise and stabilisation exercise. Immediately after treatment, disability was significantly lower in the general exercise group. No differences were found in other outcomes or at follow-up time points (48).

Psychosocial factors have an important impact on treatment outcome in NSLBP patients who also show non-acute (49) depression, pain catastrophising, fear of pain and avoidance. These aspects are part of the fear-avoidance model of chronic pain, and previous data has reported its various effects on treatment outcome (50). Pain-related fear and subsequent avoidance of movements that patients believe to be harmful can also lead to disability and physical deconditioning (51). In our study patients with a high risk of psychosocial problems are excluded based on an assessment with the Örebrö Musculoskeletal Pain Questionnaire (ÖMPSQ) (52) (53). This influential variable will be observed using the Fear-avoidance beliefs questionnaire.

This randomised controlled trial will, to our knowledge, be the first to compare two exercise programs for a well-defined subgroup of patients with NSLBP and MCI. We evaluate the effect of individualised movement control exercise versus general exercise on disability during a one year follow-up period. 


\section{Methods}

\section{Design}

In this randomised controlled trial we will include patients with non-acute NSLBP and $\mathrm{MCl}$. Patients will be recruited and treated in 5 hospital outpatient departments and 8 private practices in Switzerland. Movement control exercise will be compared to general exercise. Treatment outcomes are to be measured at baseline, post-treatment, and at 6 and 12 months follow-up. (Fig. 1)

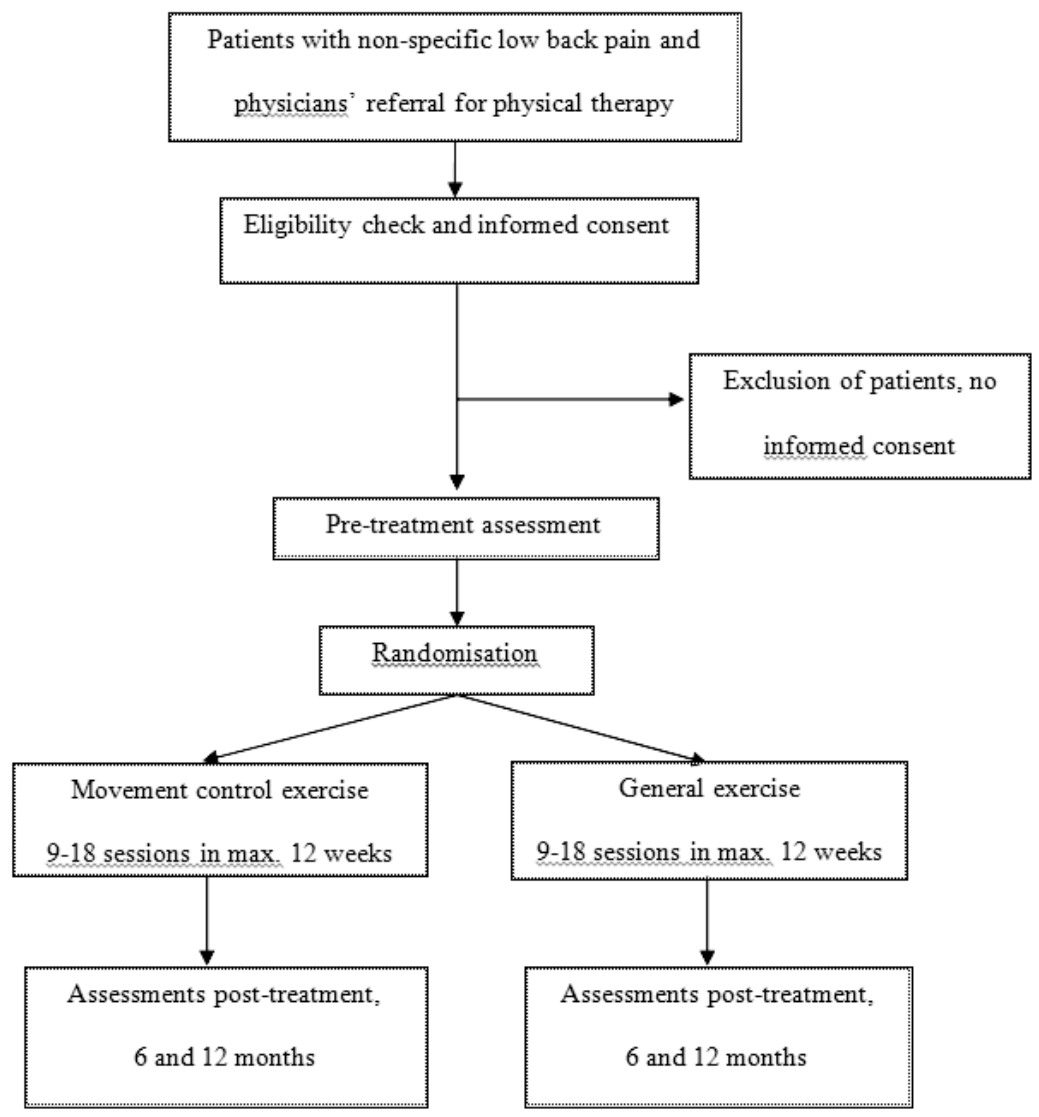

Figure 1 flow-chart of research design

Ethical approval has been granted by the Swiss Ethics Commission for Clinical Trials in the cantons of Zurich, Basel and Aargau (Switzerland). Reporting of the RCT will follow the recommendations of CONSORT Statement 2010 (www.consort-statement.org) (54). 


\section{Hypotheses}

In NSLBP patients with $\mathrm{MCl}$, treatment with movement control exercises will result in a significant decrease in disability compared to general exercise, measured with the patient specific functional scale.

In NSLBP patients with $\mathrm{MCl}$, improvement in motor control of the lumbar spine, assessed with standardised clinical tests, is associated with functional improvement and pain reduction.

\section{Participants and recruitment}

Admission criteria for the study are described in Table 2. Eligible are patients with NSLBP referred to physiotherapy by their physician. Patients will be recruited by the participating physiotherapists. Patients are included in the study if at least $2 \mathrm{MCl}$ tests are positive and if they present clinical symptoms of $\mathrm{MCl}$ as described by $(22,25,26,28,32,38)$. Patients with specific LBP, identified by the physician or by the physiotherapist are excluded from the study. To prevent floor effects in outcome measurement, a minimal disability of 5 points on the Roland and Morris Disability Questionnaire (RMQ) (55) is required. Excluded are patients with predominant psychosocial factors, defined as a score of more than 130 points on the Örebrö Musculoskeletal Pain Questionnaire (ÖMPSQ) (52) (53). An ÖMPSQ score of over 130 has been shown to correctly predict failure of return to work in $86 \%$ of cases (52). The German version of the ÖMPSQ has been used in a previous study in Switzerland $(56,57)$.

Table 2 - Inclusion and exclusion criteria

\begin{tabular}{ll}
\hline Inclusion criteria & - Age 18 to 75 years \\
& - Non-acute LBP (>6 weeks duration of symptoms) and less than 3 months of sick leave \\
& due to LBP \\
- & Two or more positive tests for impaired movement control (Luomajoki et al 2007) \\
& - At least 5 points on Roland Morris Disability questionnaire (55) \\
& - Clinical behaviour: posture and movement aggravate and ease symptoms; symptoms are \\
& relieved by reducing the strain on the lumbar region \\
& - Written informed consent \\
Exclusion criteria & - Specific LBP (Fractures, carcinoma, anomalies, nerve root affection with neurological \\
& signs e.g. sensitivity or reflex loss, muscle weakness, radicular pain below the knee) \\
& - Less than 6 weeks post-surgery following all surgery on the lower back \\
& - post-surgery with spondylodesis \\
& - high level of psychosocial risk factors (>130 points on the ÖMPQ) \\
& - Peripheral or central neurological disease \\
& - Contraindications for exercise, e.g. major cardiovascular disease or postural hypotension \\
& - Inability to understand the purpose of the study \\
& - Psychological or psychiatric problems \\
& - Chronic abuse of toxic substances such as drugs or alcohol \\
& - Use of neuroleptics, sedatives, anti-epileptics and antidepressants
\end{tabular}




\section{Baseline assessment and randomisation}

After eligibility has been confirmed, patients will be informed about the study comparing two treatments that are widely used in physiotherapy. After obtaining written informed consent, baseline measurements will be performed (see next paragraph). Participants will then be randomised using block allocation with a block size of four, to receive either movement control or general exercise. Randomisation is concealed and performed by an independent assistant at the School of Health Professions at the Zurich University of Applied Sciences via telephone.

\section{Outcome measurements}

Criteria for the selection of the outcome measurements are reliability, validity and sensitivity for statistical change.

\section{Primary outcome:}

Patient-specific LBP-related disability will be assessed using the patient specific functional scale (PSFS), a self-reported measurement for up to three individual activity limitations rated on an 11-point numeric rating scale ranging 0-10 (58). Measurement is taken at baseline, post-treatment, and at 6 and 12 months follow-up. Reliability and validity have been reported to be good (59) (60). Internal and external responsiveness are good, indicating that the test detects a change in active limitation and that this change is meaningful (61). In patients with low levels of activity limitations, the PSFS has better responsiveness than the RMQ (62).

Secondary outcomes:

General LBP-related disability will be assessed with the RMQ (55). It consists of 24 dichotomous questions to be answered with yes or no, and has a maximum disability score of 24 points. Measurement is taken at baseline, post-treatment and at 6 and 12 months follow-up. Reliability and validity have been widely tested, reliability is high, and construct and internal validity are good (63). The reliability and validity of the German version have been confirmed (49).

Pain, daily activities, social participation and professional participation will be assessed using the Graded Chronic Pain Scale (chronic pain grade = CPG) (64). The German version of the questionnaire shows significant correlations with other assessments of disability and staging of chronic pain, and a good internal consistency (Cronbach's alpha =.82) (65). Measurement is taken at baseline, post-treatment, and at 6 and 12 months follow-up. Sports and leisure activities will be assessed with a self-administered questionnaire developed for this study. 
Range of motion is measured pre- and post-treatment by finger to floor distance, a valid, reliable and responsive measurement correlating with radiography in patients with chronic low back pain (66).

Tactile acuity (two point discrimination TPD) is measured in the paravertebral lumbar region using a plastic caliper ruler between $\mathrm{L} 1$ and the iliac crest. Measurements are taken horizontally and vertically. The two point discrimination threshold is where the smallest distance at which the patient correctly reports feeling two points of the caliper instead of one is located. We will calculate the average of two procedures; one starting with an extended position of the calipers with the distance being decreased, the other starting with calipers in a contracted position and the distance being increased. Out of sequence tests are performed to avoid the recognition of a pattern. This test was recently used in patients with NSLBP (32). Reliability was not formally evaluated in this population. TPD is measured pre- and post-treatment.

Direct and indirect LBP related costs will be calculated based on the use of medication and medical treatment. Data are recorded according to information given by the patient. Indirect costs are calculated in sick leave days costs based on Swiss average salary data (Statistisches Jahrbuch der Schweiz, 2011).

\section{Treatment effect modifiers}

The following relevant covariates will be recorded in order to allow them to be controlled for in the analysis of this study. Movement control and endurance are addressed in the two treatment groups and are expected to modify treatment effect.

Movement control impairment of the lumbar spine is assessed using 6 tests described in the backgrounds section (67). Post-treatment the test will be recorded on videotape. The evaluation of video footage will be carried out by a specially trained physiotherapist who will be blinded to the treatment allocation and data from previous measurements.

Endurance of lumbar and abdominal muscles is assessed using static isometric strength tests for trunk extension and for trunk flexion pre- and post-treatment.

Fear avoidance beliefs will be measured with the self-report Fear-avoidance beliefs questionnaire for physical activities and work developed by Waddell et al $(68,69)$. The validated Swiss German Version will be used, allowing prediction of treatment outcome (68). It will be administered at baseline.

Patient's regular use of home exercises will be assessed at 6 and 12 months follow-up.

Personal characteristics (age, gender, previous episodes of back pain) will be collected at baseline. 


\section{Interventions}

Assessors and treating physiotherapists in both groups are trained for at least 4 hours and receive a manual containing descriptions of procedures and checklists. To support adherence to the treatment procedures, a structured recording form is provided. This study uses a pragmatic approach to treatment progression in both intervention groups. The therapist responsible for treatment has to select from exercises permitted for the relevant treatment group. The exercise prescription for the individual patient is determined by the clinical judgement of the therapist. Patients in each group will be treated by their specially trained physiotherapists in individual 30 minute sessions. Patients will receive 9-18 treatments within a period of 12 weeks. The number of treatments will be recorded. At least 20 minutes of each session are to be used for exercise according to the protocol. If required, a maximum of 10 minutes can be used for other physical therapy applications. Therapy will be monitored and evaluated using a therapists' treatment diary.

Patients are instructed to do at least three home exercises of either movement control or general exercise. They are strongly encouraged to continue them during the follow-up year. Patients are informed about frequency, number of repetitions and the intensity at which they are to perform the exercises.

\section{Movement control exercise group}

The patients in the movement control group will receive exercise treatment aimed at improving movement control of the lumbar spine as described in previous publications $(22,36,70)$. Patient education addresses awareness of positive and negative postural and movement related behaviour and increasing self-efficacy. Exercises are selected based on the direction of the impairment i.e. flexion, extension or frontal plane. The first step for patients is to learn to control the position and movement of the lumbar spine in different postures such as standing, squatting, 4 point kneeling and sitting. Movement control is practiced in combination with upper and lower extremity movements. In a second step, the difficulty level of exercise is increased through additional loading using long leavers or weights. If necessary, stretching/lengthening is applied after movement control has improved. Sports and strength training are allowed once good movement control is achieved.

\section{General exercise group}

Patients in this group are to be treated with the aim of improving endurance, strength and flexibility of the lumbar region. Patient education will address the importance of exercise and strength to reduce LBP. Exercises will address abdominals, erector spinae, gluteals, quadriceps and hamstrings muscle groups. The standardised exercise program starts in non-weight bearing positions and can be progressed by increasing load. Weights and resistance will be individually and progressively increased according to the guidelines 
of the American College of Sports Medicine (71). The use of equipment is not standardised and will be left to the discretion of the therapists.

\section{Main treatment contrast}

The main difference between the two treatments is the instruction of movement control in the $\mathrm{MCl}$ group, which will not be applied in the general exercise group.

\section{Sample size calculation}

Based on the results of our previous case series, inter-group difference in improvement of 0.9 points on the PSFS is to be used (72). This difference is also clinically relevant as it displays an improvement of approximately $20 \%$ compared with an expected PSFS baseline score of 5 points. We used a standard deviation of 1.5 points, alpha was set at 0.05 and the power was set at 0.9. In each group, 48 patients are needed. Anticipating a $10 \%$ drop-out rate, the required sample size was set at 106 patients to be randomised.

\section{Data analysis}

The comparability of both groups on prognostic and outcome variables at baseline will be analysed using two-sample t-tests in data with a normal distribution, Wilcoxon tests in non-parametric data and Chi-square tests in nominal data. An intention-to-treat analysis will be utilised in which all participants will be analysed in the group to which they were originally assigned. Differences between the groups over time are measured by Mann-Whitney-U-test. The influence of baseline differences on outcome measurements will be assessed in a multivariable linear regression analysis. A regression analysis of the factors being positive or negative predictors will be conducted based on covariates measured at baseline. Statistical significance is set at $p<0.05$.

\section{Blinding}

Patients and therapists cannot be blinded to treatment. To keep patients unaware of any expected treatment group benefit, patients will be informed that the effect of two wellestablished therapies is to be evaluated. An independent and blinded assessor will record videos of the movement control tests at the end of the treatment phase and perform the post-treatment physical examination. A second blinded assessor will rate the video recordings of the $\mathrm{MCl}$ tests. Statistical analysis will be blinded regarding treatment group code. The researcher who will perform the statistical analyses will not be involved in taking the measurements.

\section{Results}

Inclusion of patients began in July 2010 and is expected to last until the end of 2011. Results are expected in 2013. 


\section{Discussion}

This randomised controlled trial will compare the effectiveness of two exercise-based physiotherapy treatment protocols in a well-defined subgroup of NSLP. Inclusion is based on the clinical diagnosis of $\mathrm{MCl}$ with clinical tests shown to be reliable. The $\mathrm{MCl}$ tests allow easily applicable selection of participants. We will evaluate whether a treatment protocol addressing movement control problems is more beneficial than a general exercise protocol.

The multicentre design of the study allows treatment by different physiotherapists and improves generalisability. The selection of patients reflects the population usually found in different clinical settings. Randomisation is organised centrally and prevents selection bias.

Both treatment protocols are widely used and well-established in physiotherapy. The physiotherapists treating each group are equally instructed and experienced in applying the respective treatments. This prevents a disadvantage for the participating patients regarding group allocation.

Blinding the therapists is generally not possible. To minimise measurement bias, the measurements taken after the treatment will be taken by an assessor not involved in the treatment procedures.

The results of this study will provide evidence to improve the selection of exercise treatments for patients with NSLBP and $\mathrm{MCl}$. Results will also contribute to the understanding of the mechanisms behind $\mathrm{MCl}$ and its relation to low back pain.

\section{List of abbreviations}

NSLBP Non specific low back pain

$\mathrm{MCl} \quad$ Movement control impairment

RCT Randomised controlled trial

PSFS Patient specific functional scale

RMQ Roland Morris disability questionnaire

GCPS Graded chronic pain scale

MI Movement impairment

ÖMPSQ Örebrö musculoskeletal pain questionnaire

CPG Chronic pain grade

TPD Two point discrimination

\section{Competing interests}

The authors declare that they have no competing interests. 


\section{Authors' contributions}

$\mathrm{HL}$ originated the idea of the study, which is based on his previous work. HL, JK, JS designed the trial protocol. JS drafted the manuscript and the other authors revised it critically, corrected draft versions and approved the final manuscript.

\section{Acknowledgements}

The authors would like to acknowledge the main input of the physiotherapists and doctors in clinics and practices in Switzerland, which are: Bethesda Spital, Basel; Kantonsspital Winterthur, Winterthur; Klinik Schulthess, Zurich; Medbase Physiotherapie, Winterthur; Physio Seen, Winterthur; Physiotherapie Bellaria, Zurich; Physiotherapie Erlenbach, Erlenbach; Physiotherapie Reinach, Reinach; Physiotherapie Würenlingen, Würenlingen; Provital Physiotherapie, Egg; Rheumaklinik, Universitätsspital Zurich, Zurich; Segeten Physiotherapie, Zurich; Uniklinik Balgrist, Zurich.

We'd like to express our gratitude to Dr. Andreas Klipstein and Dr. Michael Gengenbacher for their contribution in getting ethical approval.

Further we would like to acknowledge Caroline HG Bastiaenen for her input to the research design, and Daryl Snell for his assistance in preparing the final manuscript.

The project was supported by Swiss National Science Foundation. 


\section{References}

1. Airaksinen O, Brox J, Cedraschi C, Hildebrandt J, Klaber-Moffett J, Kovacs F, et al. Chapter 4. European guidelines for the management of chronic nonspecific low back pain. Eur Spine J. 2006;15 Suppl 2:S192300. PubMed PMID: 16550448.

2. van Tulder M, Becker A, Bekkering T, Breen A, del Real MT, Hutchinson A, et al. Chapter 3. European guidelines for the management of acute nonspecific low back pain in primary care. Eur Spine J. 2006;15 Suppl 2:S169-91. Epub 2006/03/22. doi: 10.1007/s00586-006-1071-2. PubMed PMID: 16550447.

3. Dionne CE, Dunn KM, Croft PR, Nachemson AL, Buchbinder R, Walker BF, et al. A consensus approach toward the standardization of back pain definitions for use in prevalence studies. Spine (Phila Pa 1976). 2008;33(1):95-103. Epub 2008/01/01. doi: 10.1097/BRS.0b013e31815e7f94 00007632-20080101000016 [pii]. PubMed PMID: 18165754

4. Chou R, Loeser JD, Owens DK, Rosenquist RW, Atlas SJ, Baisden J, et al. Interventional therapies, surgery, and interdisciplinary rehabilitation for low back pain: an evidence-based clinical practice guideline from the American Pain Society. Spine (Phila Pa 1976). 2009;34(10):1066-77. Epub 2009/04/14. doi: 10.1097/BRS.0b013e3181a1390d. PubMed PMID: 19363457.

5. Dagenais S, Tricco AC, Haldeman S. Synthesis of recommendations for the assessment and management of low back pain from recent clinical practice guidelines. Spine J. 2010;10(6):514-29. Epub 2010/05/25. doi: S1529-9430(10)00288-3 [pii] 10.1016/j.spinee.2010.03.032. PubMed PMID: 20494814

6. Koes BW, van Tulder MW, Thomas S. Diagnosis and treatment of low back pain. BMJ. 2006;332(7555):1430-4. Epub 2006/06/17. doi: 332/7555/1430 [pii] 10.1136/bmj.332.7555.1430. PubMed PMID: 16777886; PubMed Central PMCID: PMC1479671.

7. Borkan J, Van Tulder M, Reis S, Schoene ML, Croft P, Hermoni D. Advances in the field of low back pain in primary care: a report from the fourth international forum. Spine (Phila Pa 1976). 2002;27(5):E128-32. Epub 2002/03/07. PubMed PMID: 11880849.

8. Bouter $L$, van Tulder M, Koes B. Methodologic issues in low back pain research in primary care. Spine (Phila Pa 1976). 1998;23(18):2014-20. PubMed PMID: 9779536.

9. Donelson R. Evidence-based low back pain classification. Improving care at its foundation. Eura Medicophys. 2004;40(1):37-44. Epub 2005/07/21. PubMed PMID: 16030492.

10. Billis E, McCarthy C, Oldham J. Subclassification of low back pain: a cross-country comparison. Eur Spine J. 2007;16(7):865-79. PubMed PMID: 17576604.

11. O'Sullivan P. Diagnosis and classification of chronic low back pain disorders: Maladaptive movement and motor control impairments as underlying mechanism. Manual Therapy. 2005;10(4):242-55. PubMed PMID: 69.

12. Brennan GP, Fritz JM, Hunter SJ, Thackeray A, Delitto A, Erhard RE. Identifying subgroups of patients with acute/subacute "nonspecific" low back pain: results of a randomized clinical trial. Spine (Phila Pa 1976). 2006;31(6):623-31. Epub 2006/03/17. doi: 10.1097/01.brs.0000202807.72292.a8 00007632-20060315000004 [pii]. PubMed PMID: 16540864

13. Kent $P$, Keating JL, Leboeuf-Yde $C$. Research methods for subgrouping low back pain. BMC Med Res Methodol. 2010;10:62. Epub 2010/07/06. doi: 1471-2288-10-62 [pii] 10.1186/1471-2288-10-62. PubMed PMID: 20598153; PubMed Central PMCID: PMC2908106.

14. Fersum KV, Dankaerts W, O'Sullivan PB, Maes J, Skouen JS, Bjordal JM, et al. Integration of subclassification strategies in RCTs evaluating manual therapy treatment and exercise therapy for non-specific chronic low back pain (NSCLBP): a systematic review. Br J Sports Med. 2009. Epub 2009/12/10. doi: bjsm.2009.063289 [pii] 10.1136/bjsm.2009.063289. PubMed PMID: 19996331.

15. Hayden JA, van Tulder MW, Malmivaara A, Koes BW. Exercise therapy for treatment of non-specific low back pain. Cochrane Database Syst Rev. 2005(3):CD000335. Epub 2005/07/22. doi: 10.1002/14651858.CD000335.pub2. PubMed PMID: 16034851. 
16. Hayden JA, van Tulder MW, Malmivaara AV, Koes BW. Meta-analysis: exercise therapy for nonspecific low back pain. Ann Intern Med. 2005;142(9):765-75. Epub 2005/05/04. doi: 142/9/765 [pii]. PubMed PMID: 15867409.

17. Hayden JA, van Tulder MW, Tomlinson G. Systematic review: strategies for using exercise therapy to improve outcomes in chronic low back pain. Ann Intern Med. 2005;142(9):776-85. Epub 2005/05/04. doi: 142/9/776 [pii]. PubMed PMID: 15867410.

18. Oesch P, Kool J, Hagen KB, Bachmann S. Effectiveness of exercise on work disability in patients with nonacute non-specific low back pain: Systematic review and meta-analysis of randomised controlled trials. J Rehabil Med. 2010;42(3):193-205. Epub 2010/04/23. doi: 10.2340/16501977-0524. PubMed PMID: 20411212.

19. Choi B, K. L., Verbeek Jos H, Tam Wilson W-S, Jiang Johnny Y. Exercises for prevention of recurrences of low-back pain. Cochrane Database of Systematic Reviews [Internet]. 2010; (1). Available from: http://www.mrw.interscience.wiley.com/cochrane/clsysrev/articles/CD006555/frame.html.

20. Choi Brian KL, Verbeek Jos H, Tam Wilson W-S, Jiang Johnny Y. Exercises for prevention of recurrences of low-back pain. Cochrane Database of Systematic Reviews [Internet]. 2010; (1). Available from: http://www.mrw.interscience.wiley.com/cochrane/clsysrev/articles/CD006555/frame.html.

21. Dankaerts W, O'Sullivan P, Burnett A, Straker L, Davey P, Gupta R. Discriminating healthy controls and two clinical subgroups of nonspecific chronic low back pain patients using trunk muscle activation and lumbosacral kinematics of postures and movements: a statistical classification model. Spine (Phila Pa 1976). 2009;34(15):1610-8. Epub 2009/07/01. doi: 10.1097/BRS.0b013e3181aa6175 00007632200907010-00016 [pii]. PubMed PMID: 19564772.

22. O'Sullivan PB. Masterclass. Lumbar segmental 'instability': clinical presentation and specific stabilizing exercise management. Manual Therapy. 2000;5(1):2-12. PubMed PMID: 1.

23. Vibe Fersum K, O'Sullivan PB, Kvale A, Skouen JS. Inter-examiner reliability of a classification system for patients with non-specific low back pain. Man Ther. 2009;14(5):555-61. Epub 2008/10/08. doi: S1356689X(08)00138-0 [pii] 10.1016/j.math.2008.08.003. PubMed PMID: 18838331.

24. Van Dillen LR, Sahrmann SA, Norton BJ, Caldwell CA, McDonnell MK, Bloom NJ. Movement system impairment-based categories for low back pain: stage 1 validation. The Journal of orthopaedic and sports physical therapy. 2003;33(3):126-42. PubMed PMID: 121.

25. Sahrmann SA. Diagnosis and treatment of movement impairment syndromes. Anonymous, editor. St.Louis: Mosby.; 2002.

26. Comerford MJ, Mottram SL. Functional stability re-training: principles and strategies for managing mechanical dysfunction. Manual Therapy. 2001;6(1):3-14. PubMed PMID: 5.

27. Comerford MJ, Mottram SL. Movement and stability dysfunction - contemporary developments. Manual Therapy. 2001;6(1):15-26. PubMed PMID: 6.

28. Luomajoki $\mathrm{H}$, Kool J, de Bruin ED, Airaksinen O. Reliability of movement control tests in the lumbar spine. BMC Musculoskelet Disord. 2007;8:90. Epub 2007/09/14. doi: 1471-2474-8-90 [pii]

10.1186/1471-2474-8-90. PubMed PMID: 17850669; PubMed Central PMCID: PMC2164955.

29. O'Sullivan P B, editor. Clinical instability of the lumbar spine: its pathological basis, diagnosis, and conservative management. . 3rd ed. Amsterdam, The Netherlands: Elsevier; 2004.

30. Moseley GL. I can't find it! Distorted body image and tactile dysfunction in patients with chronic back pain. Pain. 2008;140(1):239-43. Epub 2008/09/13. doi: S0304-3959(08)00444-2 [pii]

10.1016/j.pain.2008.08.001. PubMed PMID: 18786763.

31. Flor H, Diers M. Sensorimotor training and cortical reorganization. NeuroRehabilitation. 2009;25(1):19-27. Epub 2009/08/29. doi: B70P08T137N56M57 [pii] 10.3233/NRE-2009-0496. PubMed PMID: 19713616.

32. Luomajoki H, Moseley GL. Tactile acuity and lumbopelvic motor control in patients with back pain and healthy controls. Br J Sports Med. 2009. Epub 2009/06/26. doi: bjsm.2009.060731 [pii]

10.1136/bjsm.2009.060731. PubMed PMID: 19553222.

33. Solomonow M, Baratta RV, Banks A, Freudenberger C, Zhou BH. Flexion-relaxation response to static lumbar flexion in males and females. Clin Biomech (Bristol, Avon). 2003;18(4):273-9. Epub 2003/04/12. doi: S026800330300024X [pii]. PubMed PMID: 12689776. 
34. Solomonow M, Eversull E, He Zhou B, Baratta RV, Zhu MP. Neuromuscular neutral zones associated with viscoelastic hysteresis during cyclic lumbar flexion. Spine (Phila Pa 1976). 2001;26(14):E314-24. Epub 2001/07/20. PubMed PMID: 11462097.

35. Mense S. [Pathophysiology of low back pain and the transition to the chronic state - experimental data and new concepts]. Schmerz. 2001;15(6):413-7. Epub 2002/01/17. doi: 10.1007/s004820100025. PubMed PMID: 11793144.

36. Dankaerts W, O'Sullivan P, Burnett A, Straker L. Differences in sitting postures are associated with nonspecific chronic low back pain disorders when patients are subclassified. Spine (Phila Pa 1976). 2006;31(6):698-704. Epub 2006/03/17. doi: 10.1097/01.brs.0000202532.76925.d2 00007632200603150-00016 [pii]. PubMed PMID: 16540876.

37. Dankaerts W, O'Sullivan PB, Straker LM, Burnett AF, Skouen JS. The inter-examiner reliability of a classification method for non-specific chronic low back pain patients with motor control impairment. Manual Therapy. 2006;11(1):28-39. PubMed PMID: 190.

38. Luomajoki H, Kool J, de Bruin ED, Airaksinen O. Movement control tests of the low back; evaluation of the difference between patients with low back pain and healthy controls. BMC Musculoskelet Disord. 2008;9:170. Epub 2008/12/26. doi: 1471-2474-9-170 [pii] 10.1186/1471-2474-9-170. PubMed PMID: 19108735; PubMed Central PMCID: PMC2635372.

39. Luomajoki H. Movement control impairment as an output of distorted body image. International NOI congress; Nottingham, UK2010.

40. Macedo LG, Maher CG, Latimer J, McAuley JH. Motor control exercise for persistent, nonspecific low back pain: a systematic review. Phys Ther. 2009;89(1):9-25. Epub 2008/12/06. doi: ptj.20080103 [pii] 10.2522/ptj.20080103. PubMed PMID: 19056854.

41. Rasmussen-Barr E, Nilsson-Wikmar L, Arvidsson I. Stabilizing training compared with manual treatment in sub-acute and chronic low-back pain. Man Ther. 2003;8(4):233-41. Epub 2003/10/16. doi: S1356689X03000535 [pii]. PubMed PMID: 14559046.

42. Shaughnessy M, Caulfield B. A pilot study to investigate the effect of lumbar stabilisation exercise training on functional ability and quality of life in patients with chronic low back pain. Int J Rehabil Res. 2004;27(4):297-301. Epub 2004/12/02. doi: 00004356-200412000-00007 [pii]. PubMed PMID: 15572993.

43. May S. Stabilisation exercises for low back pain: a systematic review. Physiotherapy. 2008;94:179-89.

44. Ferreira P, Ferreira M, Maher C, Herbert R, Refshauge K. Specific stabilisation exercise for spinal and pelvic pain: a systematic review. Aust J Physiother. 2006;52(2):79-88. PubMed PMID: 16764545

45. Costa L, Maher C, Latimer J, Hodges P, Herbert R, Refshauge K, et al. Motor control exercise for chronic low back pain: a randomized placebo-controlled trial. Phys Ther. 2009;89(12):1275-86. PubMed PMID: 19892856.

46. Kavcic N, Grenier S, McGill SM. Quantifying tissue loads and spine stability while performing commonly prescribed low back stabilization exercises. Spine (Phila Pa 1976). 2004;29(20):2319-29. Epub 2004/10/14. doi: 00007632-200410150-00022 [pii]. PubMed PMID: 15480148.

47. Kavcic N, Grenier S, McGill SM. Determining the stabilizing role of individual torso muscles during rehabilitation exercises. Spine (Phila Pa 1976). 2004;29(11):1254-65. Epub 2004/05/29. doi: 00007632200406010-00016 [pii]. PubMed PMID: 15167666.

48. Koumantakis GA, Watson PJ, Oldham JA. Trunk muscle stabilization training plus general exercise versus general exercise only: randomized controlled trial of patients with recurrent low back pain. Phys Ther. 2005;85(3):209-25. Epub 2005/03/01. PubMed PMID: 15733046.

49. Wiesinger GF, Nuhr M, Quittan M, Ebenbichler G, Wolfl G, Fialka-Moser V. Cross-cultural adaptation of the Roland-Morris questionnaire for German-speaking patients with low back pain. Spine (Phila Pa 1976). 1999;24(11):1099-103. Epub 1999/06/11. PubMed PMID: 10361659.

50. Leeuw M, Goossens ME, Linton SJ, Crombez G, Boersma K, Vlaeyen JW. The fear-avoidance model of musculoskeletal pain: current state of scientific evidence. J Behav Med. 2007;30(1):77-94. Epub 2006/12/21. doi: 10.1007/s10865-006-9085-0. PubMed PMID: 17180640. 
51. Sieben JM, Vlaeyen JW, Portegijs PJ, Verbunt JA, van Riet-Rutgers S, Kester AD, et al. A longitudinal study on the predictive validity of the fear-avoidance model in low back pain. Pain. 2005;117(1-2):162-70. Epub 2005/08/16. doi: S0304-3959(05)00271-X [pii] 10.1016/j.pain.2005.06.002. PubMed PMID: 16099095.

52. Linton SJ, Boersma K. Early identification of patients at risk of developing a persistent back problem: the predictive validity of the Orebro Musculoskeletal Pain Questionnaire. Clin J Pain. 2003;19(2):80-6. Epub 2003/03/05. PubMed PMID: 12616177.

53. Linton SJ, Hallden K. Can we screen for problematic back pain? A screening questionnaire for predicting outcome in acute and subacute back pain. Clinical Journal of Pain. 1998;14(3):209-15. PubMed PMID: ISI:000075858700007.

54. Moher D, Hopewell S, Schulz KF, Montori V, Gotzsche PC, Devereaux PJ, et al. CONSORT 2010 Explanation and Elaboration: updated guidelines for reporting parallel group randomised trials. Brit Med J. 2010;340:. doi: ARTN c869 DOI 10.1136/bmj.c869. PubMed PMID: ISI:000276157600007.

55. Roland M, Morris R. A study of the natural history of back pain. Part I: development of a reliable and sensitive measure of disability in low-back pain. Spine. 1983;8(2):141-4. PubMed PMID: 6222486.

56. Kohlmann T, Schmidt, C. O., Pfingsten, . 2007, not published.

57. Tamcan O. Bewältigungsressourcen bei Rückenschmerzen: ein neues erhebungsinstrument für die therapeutische Praxis. Z Med Psychol. 2010;19(1):21 - 9.

58. Pengel LH, Refshauge KM, Maher CG. Responsiveness of pain, disability, and physical impairment outcomes in patients with low back pain. Spine (Phila Pa 1976). 2004;29(8):879-83. Epub 2004/04/15. doi: 00007632-200404150-00011 [pii]. PubMed PMID: 15082988.

59. Stratford P GC, Westaway M, Binkley J, . Assessing disability and change on individual patients: a report of a patient specific measure. Physiother Canada. 1995;47(4):258-63.

60. Cleland JA, Fritz JM, Whitman JM, Palmer JA. The reliability and construct validity of the Neck Disability Index and patient specific functional scale in patients with cervical radiculopathy. Spine (Phila Pa 1976). 2006;31(5):598-602. Epub 2006/03/02. doi: 10.1097/01.brs.0000201241.90914.22 00007632200603010-00019 [pii]. PubMed PMID: 16508559.

61. Beurskens AJ, de Vet HC, Koke AJ, Lindeman E, van der Heijden GJ, Regtop W, et al. A patient-specific approach for measuring functional status in low back pain. J Manipulative Physiol Ther. 1999;22(3):144-8. Epub 1999/04/30. doi: S0161475499000858 [pii]. PubMed PMID: 10220712

62. Hall AM, Maher CG, Latimer J, Ferreira ML, Costa LO. The patient-specific functional scale is more responsive than the Roland Morris disability questionnaire when activity limitation is low. Eur Spine J. 2010. Epub 2010/07/16. doi: 10.1007/s00586-010-1521-8. PubMed PMID: 20628767.

63. Roland M, Fairbank J. The Roland-Morris Disability Questionnaire and the Oswestry Disability Questionnaire. Spine (Phila Pa 1976). 2000;25(24):3115-24. Epub 2000/12/22. PubMed PMID: 11124727.

64. Latimer J, Maher CG, Refshauge K, Colaco I. The reliability and validity of the Biering-Sorensen test in asymptomatic subjects and subjects reporting current or previous nonspecific low back pain. Spine (Phila Pa 1976). 1999;24(20):2085-9; discussion 90. Epub 1999/10/30. PubMed PMID: 10543003.

65. Klasen BW, Hallner D, Schaub C, Willburger R, Hasenbring M. Validation and reliability of the German version of the Chronic Pain Grade questionnaire in primary care back pain patients. Psychosoc Med. 2004;1:Doc07. Epub 2004/01/01. PubMed PMID: 19742049; PubMed Central PMCID: PMC2736479.

66. Perret C, Poiraudeau S, Fermanian J, Colau MM, Benhamou MA, Revel M. Validity, reliability, and responsiveness of the fingertip-to-floor test. Arch Phys Med Rehabil. 2001;82(11):1566-70. Epub 2001/11/02. doi: S0003-9993(01)07692-4 [pii]

10.1053/apmr.2001.26064. PubMed PMID: 11689977.

67. Luomajoki H, Kool J, de Bruin E, Airaksinen O. Reliability of movement control tests in the lumbar spine. BMC Musculoskelet Disord. 2007;8:90. PubMed PMID: 17850669.

68. Staerkle R, Mannion AF, Elfering A, Junge A, Semmer NK, Jacobshagen N, et al. Longitudinal validation of the fear-avoidance beliefs questionnaire $(F A B Q)$ in a Swiss-German sample of low back pain patients. Eur Spine J. 2004;13(4):332-40. Epub 2004/01/10. doi: 10.1007/s00586-003-0663-3. PubMed PMID: 14714246. 
69. Waddell G, Newton M, Henderson I, Somerville D, Main CJ. A Fear-Avoidance Beliefs Questionnaire (FABQ) and the role of fear-avoidance beliefs in chronic low back pain and disability. Pain. 1993;52(2):157-68. Epub 1993/02/01. doi: 0304-3959(93)90127-B [pii]. PubMed PMID: 8455963.

70. Luomajoki H. Movement Control Impairment as a subgroup of non-specific low back pain. Institute of Clinical Medicine, School of Medicine, Faculty of Health Sciences. 22.10.2010 ed. Kuopio: Eastern Finland University Library/Sales of Publications; 2010. p. 70.

71. Whaley MH, editor. ACSM's Guidelines for exercise testing and prescription: Lippincott Williams \&Wilkins; 2006.

72. Luomajoki H, Kool J, de Bruin ED, Airaksinen O. Improvement in low back movement control, decreased pain and disability, resulting from specific exercise intervention. Sports Med Arthrosc Rehabil Ther Technol. 2010;2:11. Epub 2010/04/27. doi: 1758-2555-2-11 [pii] 10.1186/1758-2555-2-11. PubMed PMID: 20416091; PubMed Central PMCID: PMC2873574. 


\section{Appendix 1}

Luomajoki $\mathrm{H}$, Kool J, de Bruin ED, Airaksinen O: Reliability of movement control tests in the lumbar spine. BMC Musculoskelet Disord 2007, 8:90.

Test set description $1-6$

Rating description: As the subjects did not know the tests, only clear movement dysfunction was rated as "not correct". If the movement control improved by instruction and correction, it was considered that it did not infer a relevan movement dysfunction.

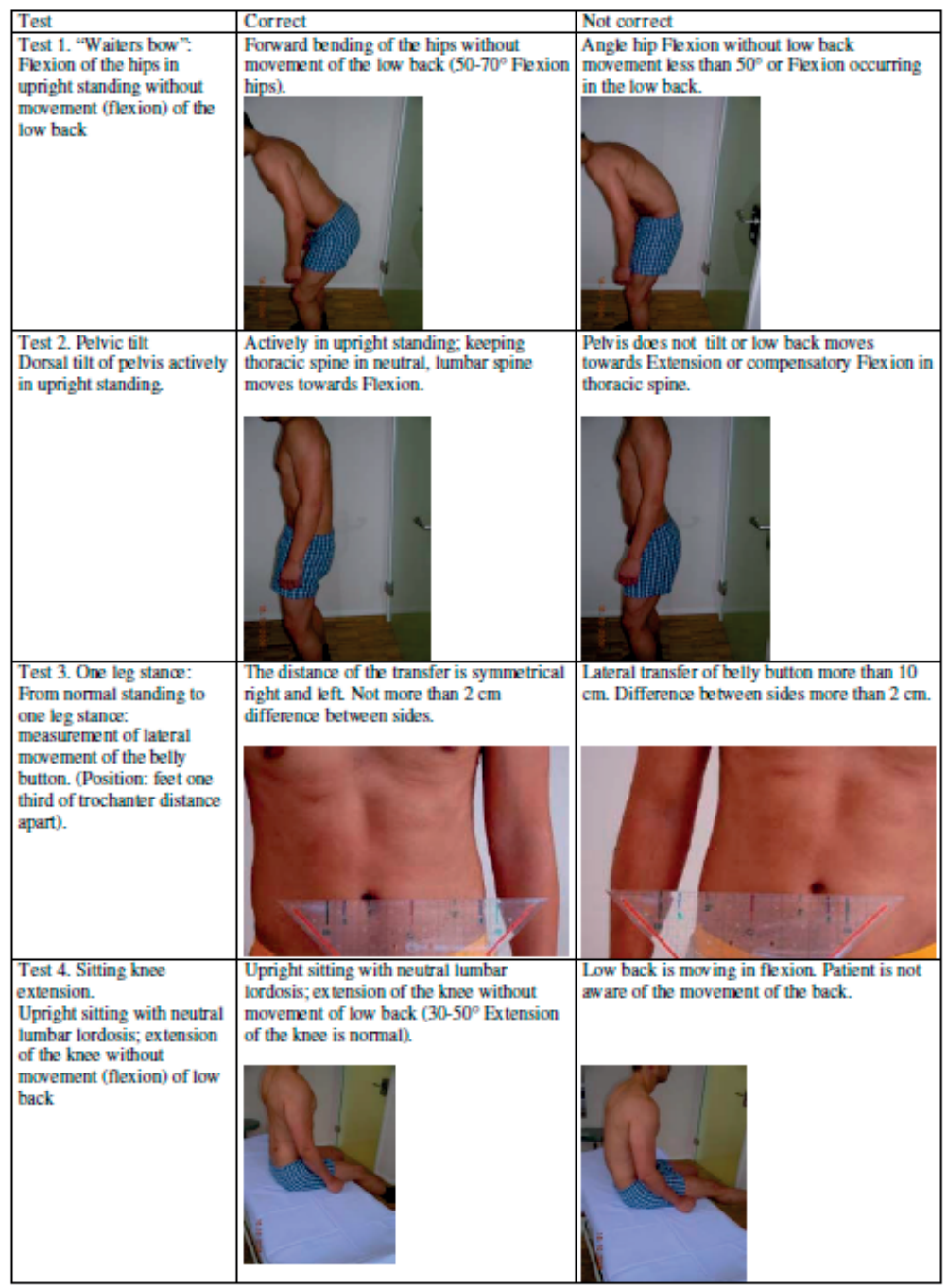




\begin{tabular}{|l|l|l|}
\hline Test 5. Quadruped position. \\
Transfer of the pelvis \\
backwards and forwards \\
("rocking") keeping low \\
back in neutral. Starting \\
position $90^{\circ}$ hip flexion. \\
the low back by transferring pelvis \\
backwards.
\end{tabular}




\section{Chapter}

\section{A tailored exercise program versus general exercise for a subgroup of patients with low back pain and movement control impairment: Short-term results of a randomised controlled trial}




\section{Abstract}

Background: Exercise is an effective treatment for patients with sub-acute and chronic low back pain (LBP). Patients with a movement control impairment $(\mathrm{MCl})$ can be diagnosed as a subgroup of patients with LBP. Unknown is which exercise intervention is most beneficial for this subgroup.

This study assessed the short-term effect of a specific exercise program targeting movement control impairment versus general exercise treatment on disability in patients with LBP and $\mathrm{MCl}$.

Methods: In a multicentre parallel group randomised controlled pragmatic trial, patients with sub-acute and chronic LBP were included. Further inclusion criteria were disability of $\geq 5$ points on the Roland-Morris Disability Questionnaire and $\geq 2$ positive tests out of a set of 6 movement control impairment tests.

A total of 106 patients were randomly assigned to either tailored movement control exercise intervention (MC, $n=52$ ) or a general exercise intervention (GE, n=54); both 9-18 individual treatment sessions, over a maximum of 12 weeks. . The primary outcome was disability measured with the Patient Specific Functional scale (PSFS). Secondary outcome was the Roland-Morris disability scale (RMDQ). Measurements were taken pre- and posttreatment.

Results: No significant difference was found following the treatment period. Baseline-adjusted between-group mean difference for the PSFS was 0.5 (SD $=0.5 ; p=0.32$ ) in favour of $\mathrm{MC}$ exercises. The Roland-Morris Disability Questionnaire revealed a significant, but not clinically relevant, between-group difference of 2.0 points $(S D=0.8 ; p=0.01)$.

Conclusion: Disability in LBP patients was reduced considerably by both interventions. However, the limited contrast between the two exercise programs may have influenced outcomes. 


\section{Introduction}

Societal cost of treatment and absence from work due to low back pain (LBP) are a major economic burden (Airaksinen et al., 2006); in Switzerland, an estimated fourteen billion Swiss Francs (15 billion US dollars) are spent annually on direct and indirect costs due to LBP. In 2007, 41\% of the working population in Switzerland reported LBP during the previous month (SNF, 2009). In most cases a specific diagnosis cannot be identified and the condition is labelled as non-specific low back pain (NSLBP)(Grob et al., 2007). Due to the heterogeneity of this patient group, it has been recommended to focus research on defining and treating subgroups (Foster et al., 2011).

Within the spectrum of NSLBP, a subgroup of patients with a movement control impairment $(\mathrm{MCl})$ can be identified. These patients present with mechanical pain, related to movement and positioning of the spine, in combination with an impairment of control during movement of the lumbar spine. To allow a more specific categorisation, the condition is further classified based on the direction of the reported control deficit, i.e. flexion, extension, frontal plane or multidirectional, as described by O'Sullivan (O'Sullivan, 2005). The rationale of $\mathrm{MCl}$ is based on the concept of repeated mechanical overload of tissues in the lower back. The clinical diagnosis of these categories showed a good reliability (Dankaerts et al., 2006; Fersum et al., 2010).

Definition and treatment of subgroups requires plausible explanations for concepts or models to underpin targeted interventions. The identification of subgroups requires clinically feasible and reliable screening procedures (Foster et al., 2011). The validity of the clinical diagnosis of the subgroup with $\mathrm{MCl}$ and its functional representation is gaining increasing support (Dankaerts \& O'Sullivan, 2011; Fersum et al., 2009). To further improve the screening procedure for $\mathrm{MCl}$, six active movement tests have been identified in a previous study which showed substantial intra- and interrater reliability and represent the clinical classification as described above (Luomajoki et al., 2007); validity of the test series was supported by research, showing that two or more positive tests, out of a total of six tests, could distinguish between patients with LBP and healthy controls (Luomajoki et al., 2008). Results of a case series, in which patients were classified as $\mathrm{MCl}$ by means of the set of six tests, showed significant improvement in disability and pain when patients were given tailored exercises that aimed to improve their control impairment (Luomajoki et al., 2010). However, the direct cause-effect relationship between $\mathrm{MCl}$ and NSLBP remains unclear.

While exercise as a treatment modality has been shown to be ineffective in the acute phase of LBP (< 6 weeks) (Airaksinen et al., 2006; Hayden et al., 2005a; Hayden et al., 2005b; Hayden et al., 2005c), several studies support the positive effect of exercise on pain and function in sub-acute and chronic pain patients (Hayden et al., 2005c). The question remains which exercises are most beneficial for which patients. Specific exercise programs to treat $\mathrm{MCl}$ are widely used in physiotherapy practice. In these programs, the impaired control is addressed with individually tailored exercises, according to the classification 
based on $\mathrm{MCl}$ tests. The postulated rationale for a treatment program for $\mathrm{MCl}$ are manifold: A positive influence on mechanically induced pain is assumed, due to a decrease of the load on nociceptive innervated tissues. Furthermore, improved activity in daily life, due to decreased disability and prevention of LBP recurrences, due to increased awareness of body positions (Kavcic et al., 2004; Moseley, 2008; Solomonow et al., 2003; Solomonow et al., 2001) may explain a positive effect. However, evidence for the effectiveness of this treatment in a healthcare setting is still lacking.

A general exercise program has previously been tested in a sub-acute and chronic population in comparison with low-load stabilisation exercises plus general exercise (Koumantakis et al., 2005). Results showed that, in the short term, disability was reduced to a greater extent by general exercise alone. However, in this study patients with all types of NSLBP were included, not only patients with NSLBP and $\mathrm{MCl}$.

Comparison of an individually tailored, specific $\mathrm{MCl}$ treatment against other active treatments for this subgroup is lacking. This article reports the short-term results of a pragmatic randomised controlled trial (RCT) in outpatient physiotherapy settings on disability in LBP patients with $\mathrm{MCl}$, testing the effectiveness of an individualised exercise program targeting $\mathrm{MCl}$ versus general exercise treatment.

\section{Methods}

\section{Trial Design}

A multicentre parallel group pragmatic RCT was executed in five hospital outpatient departments and eight private practices in Switzerland. Patients were recruited between August 2010 and February 2012 through referrals from hospitals and general practitioners, as well as through advertisements aimed at staff and students of the Zurich University of Applied Sciences, Winterthur. The study protocol has been published (Saner et al., 2011).

\section{Participants}

Patients (age 18 to 75 ) presenting with sub-acute or chronic LBP persisting longer than six weeks were invited to participate in the baseline screening and assessment procedure. Included were patients with $\mathrm{MCl}$ complaints, in which pain was provoked by movements and static positions of the spine (Mannion et al., 2010). At baseline assessment a minimum score of 5 points on the Roland-Morris Disability Questionnaire (RMDQ) was required to ensure at least a minor level of disability (Pengel et al., 2004). At least two positive tests out of six MCl tests were required for inclusion (Luomajoki et al., 2008, 2010). Patients were excluded (1) when spinal pathology was suspected or diagnosed (fractures, carcinoma, nerve root compression with neurological signs, e.g. reflex loss, muscle weakness); (2) in case of previous spinal surgery or spondylodesis; (3) if comorbid health 
conditions prohibited exercise training. (4) To focus on patients with LBP responding to movement, we excluded patients with constant pain and pain below the knee. (5) To avoid confounding with high psychosocial factors, patients with scores of more than 130 on the Örebro Musculoskeletal Pain Screening Questionnaire and more than 3 months of sick leave due to LBP, were excluded (Linton \& Boersma, 2003). For more details see flow chart Fig. 1.

\section{Randomisation and Blinding}

Once informed consent was signed, baseline assessments were taken by an independent and specially-trained physiotherapist. The randomisation schedule used to assign participants to either movement control (MC) or general exercise (GE) treatment group was generated electronically using a block size of four. Allocation to treatment of eligible patients was performed (by means of a telephone contact) by a research assistant of the university, who was not otherwise involved in the study. Blinding of participants and physiotherapists to allocation was not possible due to the nature of the treatment. Outcome assessors and data analysts were blinded to treatment allocation and were not involved in the interventions. The involved therapists and researchers were masked from the outcome measurements and trial results, except for physiotherapists in the MC group, who were informed of the results of the initial six physical $\mathrm{MCl}$ tests in order to apply $\mathrm{MCl}$ direction specific treatment.

\section{Intervention}

Participants in both groups were scheduled for treatment in the outpatient departments of the clinics and practices where they had been recruited.

Movement control (MC) treatment, as illustrated in Appendix, focused on specific active exercises to improve the individual movement control impairment as diagnosed previously. Initially, participants learned to control the impaired movements (either/and in flexion/extension/frontal plane) in closed-chain positions. They then progressed to openchain positions and to exercises with controlled movements and increased load. Postural and movement awareness was practiced in various situations. Strength training was allowed once control of pain provoking movements was achieved.

General exercise (GE) treatment, as illustrated in Appendix, followed a non-specific standardised program, in which strength and endurance of muscles of the lumbar/pelvic region and legs were exercised. All relevant muscle groups (abdominals, erector spinae, gluteals, quadriceps and hamstrings) were included in each treatment session. The progression of exercises followed the guidelines of the American College of Sports Medicine (Whaley, 2006). This exercise intervention was chosen as the control intervention because of its credibility as a treatment alternative for movement-related NSLBP (Hides \& Stanton, 2012). 
Treatment description: All participants received individual treatment sessions of 30 minutes, generally twice per week, for a maximum of 12 weeks. Recommendations of twice per week and a maximum of 12 weeks in total were chosen in line with current clinical practice (which includes 9-18 therapy sessions). This allows enough time for a comprehensive treatment which is effective in achieving optimal changes of movements and tissues.

Treatment progression followed a pragmatic approach and was determined by the clinical judgement of the physiotherapist. In each session, a timespan of maximal 10 minutes was allowed for other physiotherapy applications; duration (minutes) and types of interventions were recorded. All participants should have mastered at least three home-exercises by the end of treatment. Two regular exercise sequences per week of self-directed home-exercise were strongly recommended for the following year.

The main contrast between the two treatments was the instruction of specifically and individually tailored movement control exercises applied in the MC group, as opposed to the generalised approach in the GE group.

The physiotherapists treating the MC group were either qualified to OMT (Orthopaedic manual therapy) standards or novices working under supervision of experts at the university.

All therapists underwent at least four hours of training on the study procedures and received a manual of exercise interventions. Therapists reported on exercise programs, number of home exercises and minutes of other interventions for each patient in a written log.

\section{Baseline Descriptives}

Demographic data, including age, sex, height, weight, sports activities, workload, work status, medication usage and duration of symptoms, were documented. Descriptive measures also included the Fear-Avoidance Beliefs Questionnaire (FABQ)(Sieben et al., 2005; Staerkle et al., 2004) and the Graded Chronic Pain Scale (GCPS) version 2.0 with subscales for pain intensity, disability and state of chronicity (Turk, 2011).

\section{Measures}

Measurements were taken at baseline and after treatment (see flowchart Fig1.). The primary outcome was LBP-related disability as measured with the Patient-Specific Functional Scale (PSFS) (Hall et al., 2011; Stratford P, 1995). PSFS was chosen as the primary outcome, because it represents the clinical reality and personal relevance for patients, as it refers to the activity which is most important in limiting daily life. Additionally, clinimetric studies regarded PSFS as more responsive than RMDQ in patients with mild NSLBP (Horn et al., 2012; Pengel et al., 2004). 
The secondary outcome measure was disability, as measured with the Roland-Morris Disability Questionnaire (RMDQ). More details on the outcome measures are described in the study protocol (Saner et al., 2011).

\section{Size Calculation}

Based on a previous case series (Luomajoki et al., 2010), sample size was calculated in order to detect a mean difference between the two groups of 0.9 points on the PSFS, with an assumed standard deviation of 1.5 points. With alpha set at 0.05 and statistical power at 90\%, 48 participants were required in each group. Accounting for a drop-out rate of $10 \%, 106$ participants were included.

\section{Analysis}

Descriptive statistics of demographic data and clinical measures were performed.

Given the finding that on the PSFS the correlation between the average of three activities and the first activity mentioned by the patient was very high (0.9), the value of only the first activity was used in further analysis.

Analyses were performed according to intention-to-treat principles. Missing values after treatment were substituted by group average. For between-groups analyses, mean differences between baseline and short-term measurements, their SDs and confidence intervals were calculated, followed by a one-way between-group analysis of variance for each clinical outcome. Influences of baseline differences between the groups, and of potentially confounding variables were assessed with analysis of covariance. All analyses were conducted using IBM SPSS Statistics 20. Two-sided significance for all values was set at $p<0.05$.

Based on literature, a minimal clinically important difference for slightly disabled LBP participants was set a priori, at 30\% of baseline score for PSFS and RM (Dworkin et al., 2009; Maughan \& Lewis, 2010; Saner et al., 2011). Relative risk (RR) and number needed to treat (NNT) were calculated.

\section{Results}

\section{Participants}

A total of 201 patients were evaluated for eligibility. Reasons for exclusion $(n=48)$ are described in the flow chart in figure 1. A total of 153 patients were assessed for baseline variables, of which 47 were excluded, mainly because of not meeting minimal requirements for RMDQ $(n=24)$ and MC test series $(n=12)$. The remaining 106 participants were randomized ( $M C=52, G E=54)$. Baseline characteristics across groups (see table 1 ) were well balanced, except that the MC group had longer pain duration. 


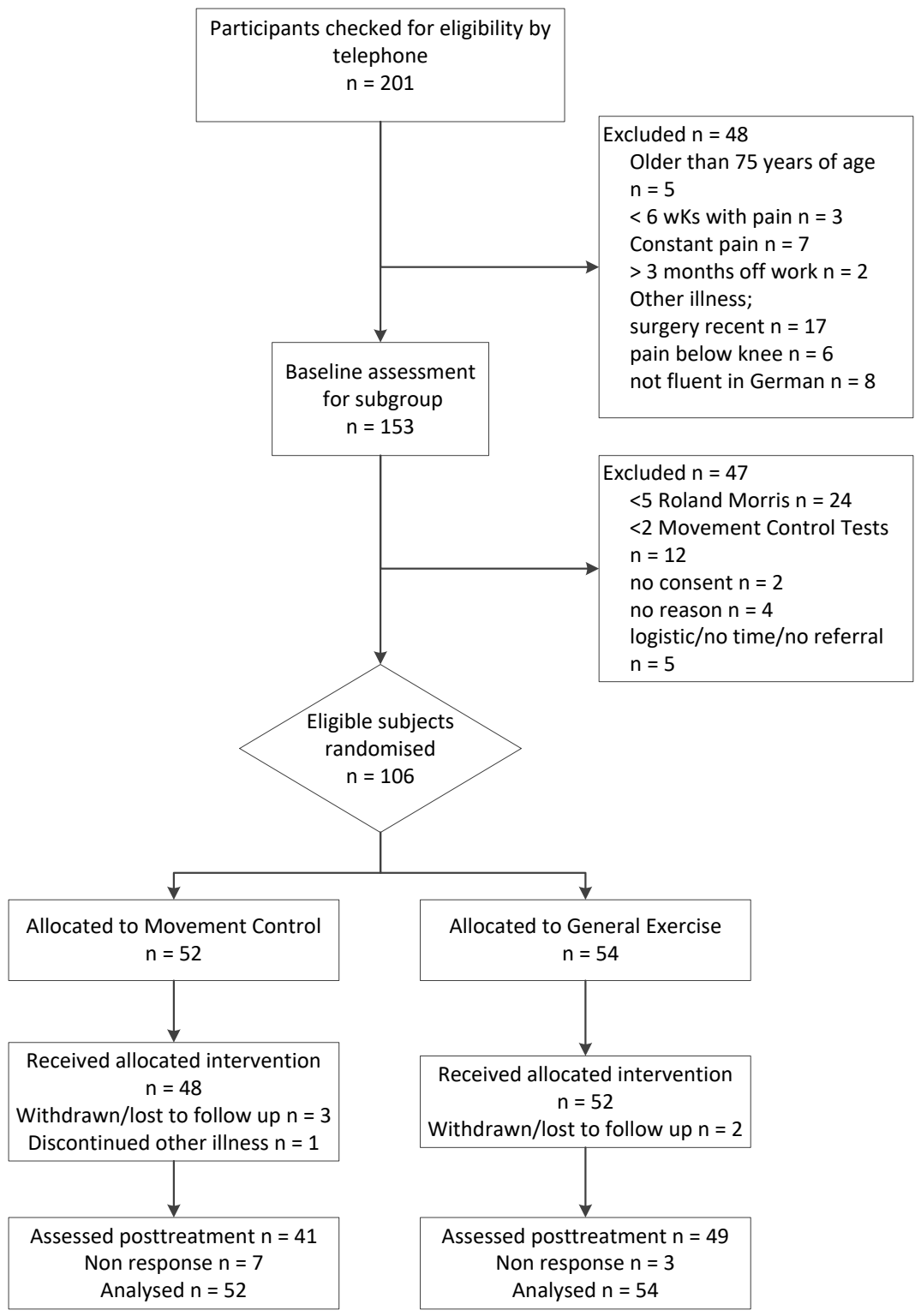

Figure 1 Flow chart of participants through the trial

Measurements after treatment were taken in 41/49 MC/GE cases respectively. Three participants in the $\mathrm{MC}$ group and two in the GE group either withdrew from the study or were lost to follow up. Seven participants in the MC group and three in the GE group finished treatment but were not assessed post-treatment. Baseline characteristics did not differ between assessed and non-assessed participants, except for the duration of pain. 
Table 1 Baseline demographic data and baseline results of questionnaires

\begin{tabular}{|c|c|c|c|c|}
\hline & & $\begin{array}{l}\text { Movement Control } \\
\mathrm{n}=52\end{array}$ & $\begin{array}{l}\text { General Exercise } \\
n=54\end{array}$ & $\begin{array}{l}\text { Total Group } \\
\mathrm{n}=106\end{array}$ \\
\hline Age (y) & mean (SD) & $42.8(13.8)$ & $40.5(14.7)$ & $41.6(14.1)$ \\
\hline Gender & female, $n$ & 16 & 24 & 40 \\
\hline Height (cm) & mean (SD) & $173.0(8.5)$ & $173.9(8.0)$ & $173.6(8.3)$ \\
\hline Weight (kg) & mean (SD) & 75.5 (11.7) & 74.7 (11.9) & $75.4(12.1)$ \\
\hline \multirow[t]{4}{*}{ Physical workload } & low, n & $23(44.2 \%)$ & $21(38.9 \%)$ & $44(41.5 \%)$ \\
\hline & medium, $\mathrm{n}$ & $22(42.3 \%)$ & $26(48.1 \%)$ & $48(45.3 \%)$ \\
\hline & heavy, $\mathrm{n}$ & $5(9.6 \%)$ & $6(11.1 \%)$ & $11(10.4 \%)$ \\
\hline & missing, $\mathrm{n}$ & $2(3.8 \%)$ & $1(1.9 \%)$ & $3(2.8 \%)$ \\
\hline \multirow[t]{4}{*}{ Sport participation } & No, n & $19(36.5 \%)$ & $16(30.8 \%)$ & 35 (33.0\%) \\
\hline & 1- $2 x$ week, n & $21(42.0 \%)$ & $23(43.1 \%)$ & $44(41.5 \%)$ \\
\hline & $>2 x$ week, $n$ & $10(20.0 \%)$ & $13(25.0 \%)$ & $23(21.7 \%)$ \\
\hline & missing, $n$ & $2(3.8 \%)$ & $2(3.8 \%)$ & $4(3.8 \%)$ \\
\hline \multirow[t]{5}{*}{ Work status } & sick leave, $\mathrm{n}$ & $1(1.9 \%)$ & $5(9.3 \%)$ & $6(5.7 \%)$ \\
\hline & part-time leave, $n$ & $3(5.8 \%)$ & $3(5.6 \%)$ & $6(5.7 \%)$ \\
\hline & full-time, $n$ & 39 (75.0\%) & $38(70.4 \%)$ & 77 (72.6\%) \\
\hline & no paid job, $n$ & $5(9.6 \%)$ & $8(14.8 \%)$ & $13(12.3 \%)$ \\
\hline & missing, $n$ & $4(7.7 \%)$ & 0 & $4(3.8 \%)$ \\
\hline \multirow[t]{5}{*}{ Pain Duration* (y) } & mean (SD) & $11.6(12.8)$ & $8.4(8.9)$ & $10.0(11.0)$ \\
\hline & $<1$ year, $\mathrm{n}$ & 4 & 8 & $12(11.3 \%)$ \\
\hline & $1-5$ years, $n$ & 17 & 15 & $32(30.2 \%)$ \\
\hline & $>5$ years, $\mathrm{n}$ & 26 & 27 & $53(50.0 \%)$ \\
\hline & missing, $\mathrm{n}$ & 5 & 4 & $9(8.5 \%)$ \\
\hline ÖMPQ & mean (SD) & $78.3(24.3)$ & $81.5(20.4)$ & $79.9(22.3)$ \\
\hline \multirow[t]{3}{*}{ FABQ Total } & mean (SD) & $29.9(13.7)$ & $34.7(15.4)$ & $32.3(14.7)$ \\
\hline & missing, $n$ & $2(3.8 \%)$ & $3(5.6 \%)$ & $5(4.7 \%)$ \\
\hline & mean (SD) & $25.9(9.8)$ & $29.5(11.0)$ & $27.8(10.4)$ \\
\hline GCPS Pain Intensity score & mean (SD) & $14.7(4.7)$ & $16.5(4.2)$ & $15.6(4.5)$ \\
\hline GCPS Disability score & mean (SD) & $11.5(7.2)$ & $13.3(8.3)$ & $12.4(7.6)$ \\
\hline \multirow[t]{5}{*}{ GCPS Chronic Pain score } & Grade I, n & $21(41.2 \%)$ & $16(30.8 \%)$ & 37 (35.9\%) \\
\hline & Grade II, n & $17(33.3 \%)$ & $20(38.5 \%)$ & 37 (35.9\%) \\
\hline & Grade III, n & $10(19.6 \%)$ & $11(21.2 \%)$ & $21(20.4 \%)$ \\
\hline & Grade IV, n & $3(5.9 \%)$ & $5(9.6 \%)$ & $8(7.8 \%)$ \\
\hline & missing, $\mathrm{n}$ & $1(1.9 \%)$ & $2(3.7 \%)$ & $3(2.8 \%)$ \\
\hline
\end{tabular}

* Overall duration of LBP

ÖMPQ: Oerebrö musculoskeletal pain questionnaire (Linton \& Boersma, 2003)

FABQ: Fear-avoidance beliefs questionnaire - 0 (low fear); 96 (highest fear) (Sieben et al., 2005; Staerkle et al., 2004)

GCPS: Graded Chronic Pain Scale: subscale for pain (0-30); subscale for disability (0-40); subscale for pain grade (0-IV;0=no pain, no disability; IV=high disability) (Turk, 2011) 


\section{Adherence}

Seventy-nine (74.5\%) treatment logs ( $M C=41 ; G E=38)$ were returned by physiotherapists for analysis. The mean number (SD) of treatment sessions for $M C$ and GE groups was 8.4(2.9) and 8.8(3.4) respectively; the number of home exercises recommended at end of treatment was 3.9(1.3) and 5.0(2.7), respectively. Participants in both groups started with a mean number of impaired movement control tests of 3.9; after treatment the MC group improved to a mean of 1.8 and the GE participants to 2.8 positive tests.

\section{Primary outcome}

There was no statistically significant difference on the PSFS between the two exercise groups after treatment ( $p=0.32$; see table 2 ). Based on literature and baseline differences, pain intensity, pain duration and baseline PSFS were tested for their influence on group differences (primary outcome) using univariate analysis of variance. The baseline PSFS was identified as a potentially influencing factor $(p<0.001)$. Analysis of covariance (ANCOVA) showed no significant change on the primary outcome, however.

Table 2 Results of disability scores pre- and post-treatment and between-group differences

\begin{tabular}{|c|c|c|c|c|c|}
\hline \multirow[b]{2}{*}{ Outcomes } & \multicolumn{2}{|c|}{ Pre-treatment } & \multicolumn{2}{|c|}{ Post-treatment } & \multirow{2}{*}{$\begin{array}{l}\text { Difference between groups } \\
\text { post-treatment (change scores } \\
\text { pre-post-treatment) } \\
\text { Mean (SD, 95\%Cl)p-value }\end{array}$} \\
\hline & $\begin{array}{l}M C \\
(n=52) \\
\text { mean (SD) }\end{array}$ & $\begin{array}{l}\mathrm{GE} \\
(n=54) \\
\text { mean (SD) }\end{array}$ & $\begin{array}{l}\text { MC } \\
(n=52) \\
\text { mean (SD) }\end{array}$ & $\begin{array}{l}\text { GE } \\
(n=54) \\
\text { mean (SD) }\end{array}$ & \\
\hline PSFS activity1 & $4.3(1.9)$ & $4.0(2.0)$ & $7.4(1.9)$ & $6.6(2.2)$ & $0.5(0.5)-0.5-1.5 \quad 0.32$ \\
\hline RMDQ & $9.1(3.2)$ & $8.2(3.3)$ & $4.2(3.7)$ & $5.2(4.0)$ & $2.0(0.8) 0.4-3.5 \quad 0.01^{* *}$ \\
\hline
\end{tabular}

MC: Movement control group GE: General exercise group; PSFS: Patient-Specific Functional Scale self-score activity mentioned first, ranging from 0 (unable to perform) to 10 (able to perform without difficulties); RMDQ: Roland-Morris Disability Questionnaire score, ranging from 0 (no difficulties) to 24 (extreme difficulties); SD: standard deviation; $\mathrm{Cl}$ : confidence interval

\section{Secondary outcome}

For the RM a significant difference in mean change scores between the two groups was found (mean=2.0; 95\% Cl=0.4-3.5; $p=0.01$ ).

\section{Minimal Clinically Important Difference}

The minimal clinically important difference, defined a priori as $30 \%$ of the baseline scores, was 1.26 and 2.7 for the PSFS and RM respectively (Dworkin et al., 2009). Concerning the absolute number of participants with a clinically significant change, no significant difference could be found at follow-up for both variables (Table 2). However, as table 3 shows, relative risk, the factor reflecting improvement, differed in favour of the $\mathrm{MC}$ group for 
both outcomes with PSFS/RM;1.25/1.39 respectively. The average number of participants who need to be treated to provide improvement with $M C$ is shown as numbers needed to treat (PSFS/RM;7/5).

Table 3 Patients with a minimal clinically important difference post-treatment; relative risk and number needed to treat (NNT) for clinical important improvement

\begin{tabular}{|c|c|c|c|c|c|}
\hline Outcomes & $\begin{array}{l}\text { Total group }(n=106) \\
n(\%)\end{array}$ & $\begin{array}{l}\text { MC group }(n=52) \\
n(\%)\end{array}$ & $\begin{array}{l}\text { GE group }(n=54) \\
n(\%)\end{array}$ & Relative risk $(95 \% \mathrm{Cl})$ & NNT \\
\hline PSFS & $73(68.9)$ & $40(76.9)$ & $33(61.1)$ & $1.25(0.97-1.42)$ & 7 \\
\hline RMDQ & $70(66.0)$ & $40(76.9)$ & $30(55.6)$ & 1.39 (1.04-1.83) & 5 \\
\hline
\end{tabular}

MC: Movement control group; GE: General exercise group; PSFS: Patient-Specific Functional Scale; RMDQ: Roland-Morris Disability Questionnaire; Cl: confidence interval; NNT: numbers needed to treat

\section{Co-interventions}

Four participants ( 1 in GE and 3 in MC) reported additional self-financed treatments (acupuncture, massage) during the treatment period.

\section{Work Status and Return to Work}

Of the 12 participants who were on sick leave due to LBP at baseline, eight returned to unrestricted work post-treatment. One participant in the GE group (id73) remained on restricted work status. Three participants who were initially on sick leave withdrew from the study for personal reasons. Work status post-treatment was not reported by three participants.

\section{Discussion}

This multicentre randomised controlled trial is, as far as we know, the first study to uniquely include NSLBP participants with $\mathrm{MCl}$. Differences in primary outcome between the $M C$ and GE groups were small and insignificant. Only between-group differences in improvements on the Roland-Morris questionnaire, the secondary outcome, reached significance. Both groups improved substantially in all treatment outcomes. Given the positive natural course of NSLBP, this improvement was to be expected.

\section{Methodological Limitations}

Several limitations of this study must be noted. Since recruitment from participating practices was below expectations, thirty participants were recruited among staff and students of the university through an additional internal advertisement. These participants were not referred by a physician. However, analysis showed that these participants were comparable to referred patients for baseline characteristics and outcomes. 
Sixteen participants were not assessed post-treatment. Six withdrew from the study or developed other illnesses. Final assessment after participants had completed their treatment was not possible in ten cases for logistic reasons, since the procedure required an additional consultation with an independent assessor.

To improve recruitment from participating physiotherapists, the protocol allowed for a maximum of ten minutes per treatment session to be used for other techniques, such as muscle stretching and mobilisation. Log books showed a wide variety of additional interventions, which was comparable in both groups. Our research aim was to evaluate, whether for a specific subgroup of patients with $\mathrm{MCl}$, a $\mathrm{MCl}$ intervention is more effective than a general exercise physical therapy program. These strict design choices may have diminished the treatment contrast between groups. For example, a comparison of $\mathrm{MCl}$ exercise versus usual care by a physician, prescribing physical therapy only in a few cases, might yield larger between-group differences. However, the design used for this RCT to our opinion best reflects current treatment choices in everyday physical therapy practice.

Other possible influences on the results could originate from treatment frequency and number of treatment sessions. A treatment frequency of 2 sessions per week was a recommendation rather than a rule; the number of sessions was reported in the log book and showed no difference between the groups. Similarly the number of home exercises is comparable in both groups. Controlling the quality of and adherence to exercise during the treatment phase was up to the physiotherapist. However, we think the influences are comparable in both groups and we have no reason not to believe the reports of patients or therapists.

\section{Theoretical and Clinical Implications}

We used two measures of disability (PSFS, RMDQ). PSFS did not show significant between-group differences, while difference in RMDQ was significant. The PSFS was chosen as primary outcome, because in previous research sensitivity to change was reported to be higher than for the RMDQ in participants with low levels of disability (Beurskens et al., 1999; Pengel et al., 2004). With the PSFS the patient individually selects his limited activities, which we expected to be more responsive for disability outcome.

The RMD questionnaire, on the other hand, assesses the disability over the past 48 hours using 24 standard items, to be valued in a dichotomous manner. With a mean of eight to nine points at baseline, our patient sample was only mildly affected. Although a floor effect could be expected, the short term between-group difference in RMDQ improvement was significant in favour of the MC group.

We had planned to evaluate disability using the average of three PSFS activities. However, several participants were unable to name three activities. A frequent reason was, that participants mentioned activities that were not currently performed (snow shovelling or digging the garden), making these activities unsuitable for the assessment of disability during the past week. Therefore, we assessed correlations and found a very high 
correlation (0.9) between the first activity mentioned and the average of the maximal three activities. To ease the evaluation process, we proceeded with only the first activity mentioned in all further analysis.

Between-group differences for the minimal clinically important change were smaller than the a priori set level of $30 \%$. However, on evaluation of participants with a minimal clinically important change of improvement, $77 \%$ of participants within the MC group improved more than the determined 30\% for both outcomes. The general exercise group improved $61 \%$ for PSFS and 55\% for RMDQ above minimal clinical importance. This resulted in numbers needed to treat of $7 / 5$ patients in favour of the movement control treatment, when measured with PSFS or RMDQ respectively.

This study showed that in the short-term, movement control exercise was not significantly superior to general exercise for patients with $\mathrm{MCl}$. One reason could be the low contrasts between the two treatment programs. Although MC and GE exercises were distinctly different at the beginning of the intervention, there was an increasing similarity of exercises between the two programs as treatment progressed. MC treatment progression shifted from closed chain to open chain exercises, with incremental loading, introducing strengthening components. GE treatment focused on strength without specifically paying attention to movement control impairments.

A second explanation for the non-significant differences in outcome may be that, in patients with positive $\mathrm{MCl}$ tests, the loss of movement control is not the only factor contributing to NSLBP. Treatments in this study focused on the physical functional impairment of movement. Through the addition of cognitive characteristics to subgroup definition and treatment, as recently proposed, predictive models and patient outcomes may improve (O'Sullivan, 2012).

\section{Theoretical background}

The physiological and cognitive rationales for movement control exercise have not been widely validated and are based on smaller studies. The physiological rationale of reducing mechanical overload on connective tissue, caused by uncontrolled movements, has been proposed by Kavic (Kavcic et al., 2004). In the current study, the attempt to control the load through improved muscular coordination, which resulted in a reduction in the number of positive $\mathrm{MCl}$ tests and improved disability, was achieved with both types of intervention.

The intervention using MC exercises also addressed body and movement awareness, positive experiences with pain-free movement and explanations for the improved movement control. These cognitive aspects may have had a potential impact on the reorganisation of the cerebral cortex representing areas of the low back and on the reversal of central sensitisation processes in the nervous system (Nijs et al., 2010; Voss et al., 2010; Wand et al., 2011). However, questions about changes in the nervous system could not be answered in the current study. 


\section{Conclusion}

This randomized controlled trial, comparing 9-12 weeks of specific movement control training to general exercise, showed no difference in improvement of LBP disability. Future research should extend the criteria for the definition of clinical subgroups among patients with LBP, as well as further develop treatments focused on these subgroups. Distinctly different exercise programs are essential in future investigations concerning the question, "Which exercise is best for whom?". We recommend longitudinal studies to explore cognitive changes in patients with impaired movement control and NSLBP as well as treatment-induced biological changes.

\section{Acknowledgements}

The authors wish to thank the physiotherapists, doctors and patients participating in this study. Without the immense support of clinicians, this study would not have been possible. Special acknowledgement and sincere thanks to Greet Mommen (MSc., scientific illustrator at Maastricht University, Dept. of Anatomy \& Embryology) for the illustrations of exercises in the appendix.

\section{Declaration of interest}

The project was supported by the Swiss National Science Foundation (SNSF). (Project no. 127240)

The trial was registered (ISRCTN80064281) and ethical approval obtained from the Swiss Ethics Committee KEK-ZH-NR: 2010-0034/5. The protocol has been published previously (Saner et al., 2011). 


\section{References}

Airaksinen O, Brox J, Cedraschi C, Hildebrandt J, Klaber-Moffett J, Kovacs F, Mannion A, Reis S, Staal J, Ursin H, Zanoli G 2006 Chapter 4. European guidelines for the management of chronic nonspecific low back pain. Eur Spine J 15 Suppl 2, S192-300.

Beurskens AJ, de Vet HC, Koke AJ, Lindeman E, van der Heijden GJ, Regtop W, Knipschild PG 1999 A patient-specific approach for measuring functional status in low back pain. J Manipulative Physiol Ther 22, 144-148.

Dankaerts W, O'Sullivan P 2011 The validity of O'Sullivan's classification system (CS) for a sub-group of NS-CLBP with motor control impairment $(\mathrm{MCl})$ : overview of a series of studies and review of the literature. Man Ther 16, 9-14.

Dankaerts W, O'Sullivan PB, Straker LM, Burnett AF, Skouen JS 2006 The inter-examiner reliability of a classification method for non-specific chronic low back pain patients with motor control impairment. Manual Therapy 11, 28-39.

Dworkin RH, Turk DC, McDermott MP, Peirce-Sandner S, Burke LB, Cowan P, Farrar JT, Hertz S, Raja SN, Rappaport BA, Rauschkolb C, Sampaio C 2009 Interpreting the clinical importance of group differences in chronic pain clinical trials: IMMPACT recommendations. Pain 146, 238-244.

Fersum KV, Dankaerts W, O'Sullivan PB, Maes J, Skouen JS, Bjordal JM, Kvale A 2009 Integration of sub-classification strategies in RCTs evaluating manual therapy treatment and exercise therapy for non-specific chronic low back pain (NSCLBP): a systematic review. Br J Sports Med.

Fersum KV, Dankaerts W, O'Sullivan PB, Maes J, Skouen JS, Bjordal JM, Kvale A 2010 Integration of subclassification strategies in randomised controlled clinical trials evaluating manual therapy treatment and exercise therapy for non-specific chronic low back pain: a systematic review. Br J Sports Med 44, 1054-1062.

Foster NE, Hill JC, Hay EM 2011 Subgrouping patients with low back pain in primary care: are we getting any better at it? Man Ther 16, 3-8.

Grob D, Frauenfelder H, Mannion AF 2007 The association between cervical spine curvature and neck pain. Eur Spine J 16, 669-678.

Hall AM, Maher CG, Latimer J, Ferreira ML, Costa LO 2011 The patient-specific functional scale is more responsive than the Roland Morris disability questionnaire when activity limitation is low. Eur Spine J 20, 79-86.

Hayden JA, van Tulder MW, Malmivaara A, Koes BW 2005a Exercise therapy for treatment of non-specific low back pain. Cochrane Database Syst Rev, CD000335.

Hayden JA, van Tulder MW, Malmivaara AV, Koes BW 2005b Meta-analysis: exercise therapy for nonspecific low back pain. Ann Intern Med 142, 765-775.

Hayden JA, van Tulder MW, Tomlinson G 2005c Systematic review: strategies for using exercise therapy to improve outcomes in chronic low back pain. Ann Intern Med 142, 776-785.

Hides J, Stanton W 2012 Muscle imbalance among elite Australian rules football players: a longitudinal study of changes in trunk muscle size. J Athl Train 47, 314-319.

Horn KK, Jennings S, Richardson G, Vliet DV, Hefford C, Abbott JH 2012 The patient-specific functional scale: psychometrics, clinimetrics, and application as a clinical outcome measure. J Orthop Sports Phys Ther 42, 30-42.

Kavcic N, Grenier S, McGill SM 2004 Determining the stabilizing role of individual torso muscles during rehabilitation exercises. Spine (Phila Pa 1976) 29, 1254-1265.

Koumantakis GA, Watson PJ, Oldham JA 2005 Trunk muscle stabilization training plus general exercise versus general exercise only: randomized controlled trial of patients with recurrent low back pain. Phys Ther 85, 209225.

Linton SJ, Boersma K 2003 Early identification of patients at risk of developing a persistent back problem: the predictive validity of the Orebro Musculoskeletal Pain Questionnaire. Clin J Pain 19, 80-86.

Luomajoki H, Kool J, de Bruin ED, Airaksinen O 2007 Reliability of movement control tests in the lumbar spine. BMC Musculoskelet Disord 8, 90.

Luomajoki H, Kool J, de Bruin ED, Airaksinen O 2008 Movement control tests of the low back; evaluation of the difference between patients with low back pain and healthy controls. BMC Musculoskelet Disord 9, 170. 
Luomajoki H, Kool J, de Bruin ED, Airaksinen O 2010 Improvement in low back movement control, decreased pain and disability, resulting from specific exercise intervention. Sports Med Arthrosc Rehabil Ther Technol 2,11 .

Mannion AF, Caporaso F, Pulkovski N, Sprott H 2010 Goal attainment scaling as a measure of treatment success after physiotherapy for chronic low back pain. Rheumatology (Oxford) 49, 1734-1738.

Maughan EF, Lewis JS 2010 Outcome measures in chronic low back pain. Eur Spine J 19, 1484-1494.

Moseley GL 2008 I can't find it! Distorted body image and tactile dysfunction in patients with chronic back pain. Pain 140, 239-243.

Nijs J, Van Houdenhove B, Oostendorp RA 2010 Recognition of central sensitization in patients with musculoskeletal pain: Application of pain neurophysiology in manual therapy practice. Man Ther 15, 135-141.

O'Sullivan P 2005 Diagnosis and classification of chronic low back pain disorders: Maladaptive movement and motor control impairments as underlying mechanism. Manual Therapy 10, 242-255.

O'Sullivan P 2012 It's time for change with the management of non-specific chronic low back pain. Br J Sports Med 46, 224-227.

Pengel LH, Refshauge KM, Maher CG 2004 Responsiveness of pain, disability, and physical impairment outcomes in patients with low back pain. Spine (Phila Pa 1976) 29, 879-883.

Saner J, Kool J, de Bie RA, Sieben JM, Luomajoki H 2011 Movement control exercise versus general exercise to reduce disability in patients with low back pain and movement control impairment. A randomised controlled trial. BMC Musculoskelet Disord 12, 207.

Sieben JM, Vlaeyen JW, Portegijs PJ, Verbunt JA, van Riet-Rutgers S, Kester AD, Von Korff M, Arntz A, Knottnerus JA 2005 A longitudinal study on the predictive validity of the fear-avoidance model in low back pain. Pain $117,162-170$.

SNF SN 2009 Muskuloskelettale Gesundheit - Chronische Schmerzen, NFP 53, Synthesebericht der Leitungsgruppe. Schweizerischer Nationalfonds SNF Abteilung IV, Orientierte Forschung.

Solomonow M, Baratta RV, Banks A, Freudenberger C, Zhou BH 2003 Flexion-relaxation response to static lumbar flexion in males and females. Clin Biomech (Bristol, Avon) 18, 273-279.

Solomonow M, Eversull E, He Zhou B, Baratta RV, Zhu MP 2001 Neuromuscular neutral zones associated with viscoelastic hysteresis during cyclic lumbar flexion. Spine (Phila Pa 1976) 26, E314-324.

Staerkle R, Mannion AF, Elfering A, Junge A, Semmer NK, Jacobshagen N, Grob D, Dvorak J, Boos N 2004 Longitudinal validation of the fear-avoidance beliefs questionnaire (FABQ) in a Swiss-German sample of low back pain patients. Eur Spine J 13, 332-340.

Stratford P GC, Westaway M, Binkley J, 1995 Assessing disability and change on individual patients: a report of a patient specific measure. Physiother Canada 47, 258-263.

Turk DC, Melzack, R. 2011 Handbook of pain assessment. The Guildford Press.

Voss MW, Prakash RS, Erickson KI, Basak C, Chaddock L, Kim JS, Alves H, Heo S, Szabo AN, White SM, Wojcicki TR, Mailey EL, Gothe N, Olson EA, McAuley E, Kramer AF 2010 Plasticity of brain networks in a randomized intervention trial of exercise training in older adults. Front Aging Neurosci 2.

Wand BM, Parkitny L, O'Connell NE, Luomajoki H, McAuley JH, Thacker M, Moseley GL 2011 Cortical changes in chronic low back pain: current state of the art and implications for clinical practice. Man Ther 16, 15-20.

Whaley MH 2006 ACSM's Guidelines for exercise testing and prescription, in: Medicine ACoS (Ed.). Lippincott Williams \&Wilkins. 
Chapter

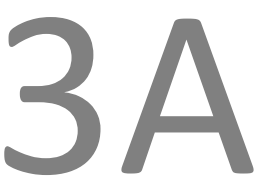

Supplement Exercise Procedures 


\section{Exercise procedures for $M C$ treatment}

Exercises depend on the initially classified impairment. Home exercises depend on the stage of progression in the physiotherapy treatment and are recommended from several times per day initially to at least twice per week over one year.

\section{Flexion impairment}

\section{0-70 degrees}

(1)

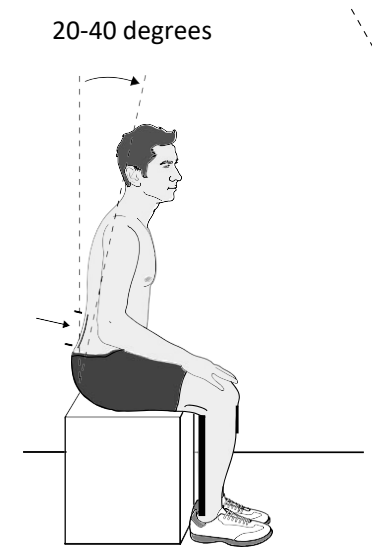

A

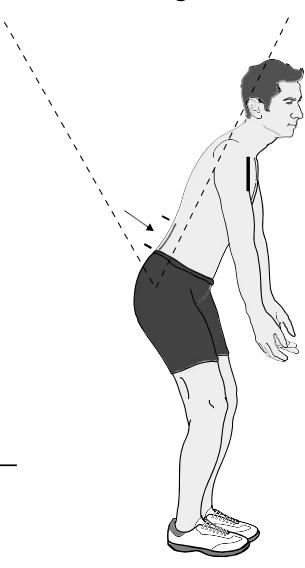

B

C

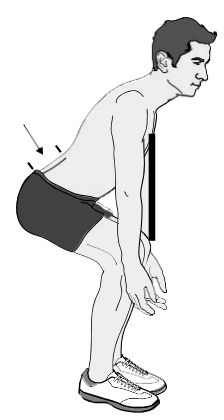

If "waiter's bow" is incorrectly performed:

The patient is asked to move his upper body forward by flexion of the hip (50 - 70 degrees) without flexion of the lumbar spine. Initially in sitting (alternatively lean on table with hands). As progression change to standing or in combination with squatting. A tape adds proprioceptive input.

(2) $\mathbf{A}$

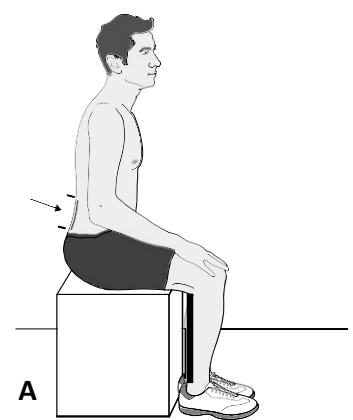

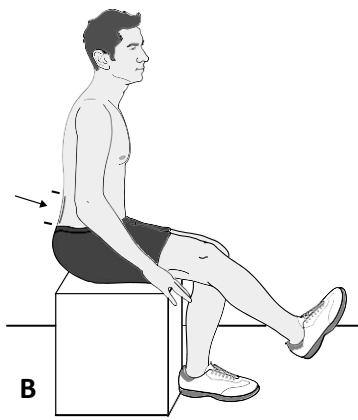

If "sitting straight leg" is incorrectly performed:

The patient learns to find his "neutral" position in sitting. He is asked to straighten one knee, as far as he notices that the spine wants to move as well. 
(3)

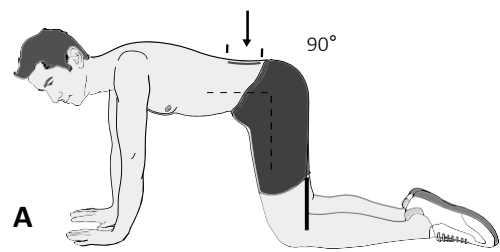

B

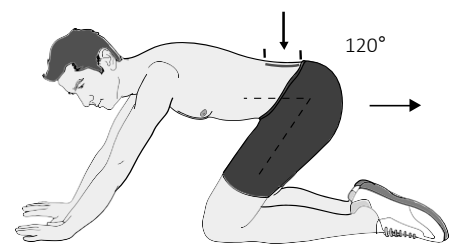

If "rocking backwards" is incorrectly performed:

Initial positioning in "neutral position" with 90 degree hip flexion and a slightly curved lumbar spine. The patient is then asked to move the pelvis backwards and keep the position of the spine. 120 degrees of hip flexion without movement of the lumbar spine is the expected range.

\section{Extension impairment}

(4) $\mathbf{A}$

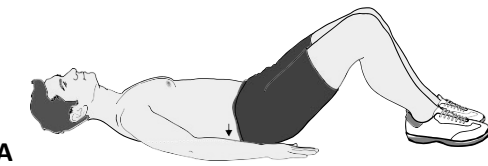

B

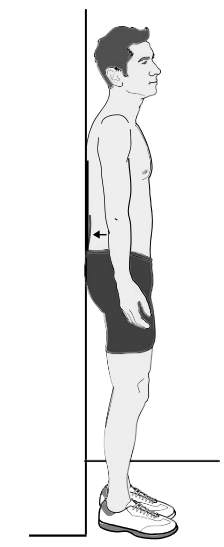

C

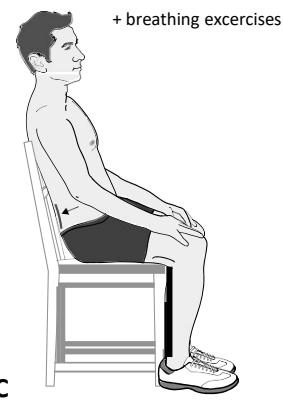

If the "pelvic tilt" is performed incorrectly:

Initially the patient learns to roll back the pelvis in supine. Progression includes standing against a wall with decreasing distance of the heel to the wall. Or sitting in a chair with flexed lumbar spine combined with breathing exercises (specially for patients with an active extension impairment). To be able to tilt the pelvis without bending the knees in free standing is expected. For a long term standing position the patient learns the position in neutral. 

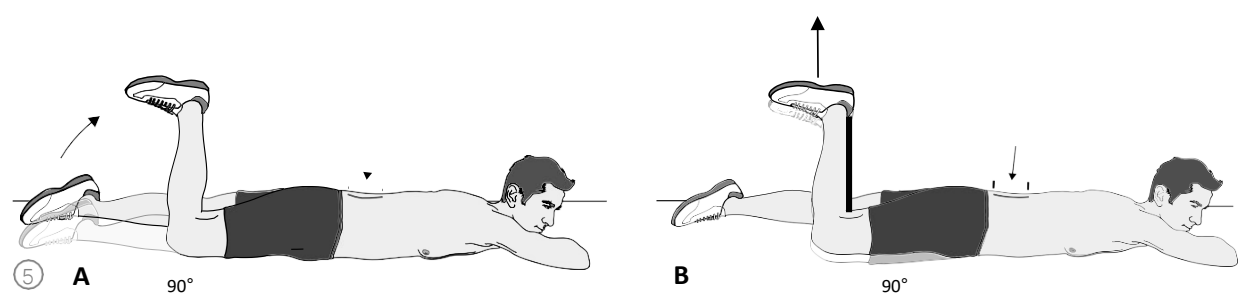

If "prone knee bend" is performed incorrectly:

Initially the patient learns the "neutral" position of the lumbar spine. Bending the knee in prone, bending and lifting the flexed knee lifting with the extended knee are the progressions. This exercise can be progressed to standing with and without support by leaning on hands.

(6) $\mathbf{A}$

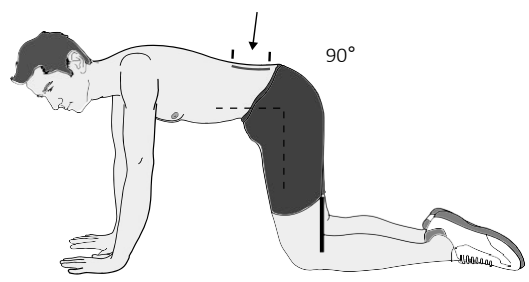

If "rocking forward" is performed incorrectly:

As in all positions the "neutral "positioning in four-point kneeling is the first phase. The trunk is then moved forward along the longitudinal axis. 60 degrees of hip flexion without movement in the lumbar spine is the target. 


\section{(7)}

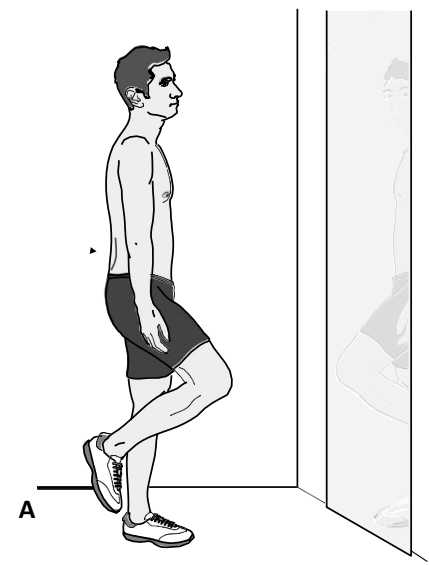

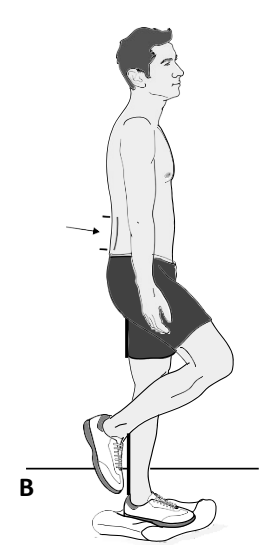

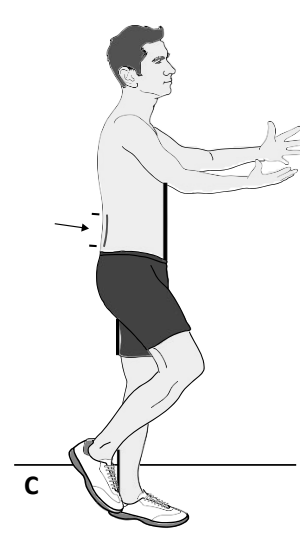

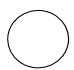

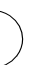

(19)

If "one leg stance" is performed incorrectly:

Initially the patient is standing in front of a mirror and corrects his trunk position while unloading/lifting one leg. As the patients is able to perform symmetrical movements, the impaired side progresses to single leg exercises on uneven surfaces or combined with dual task performance.

\section{Multidirectional impairment}

Patients in this category typically show difficulties of finding a "neutral" lumbar spine position in any condition. Movements which are connected to pain may be exercised primarily. How much support a patient needs is up to the individual performance in the testing procedure. 


\section{Exercise procedures for GE treatment}

All exercises are included in every treatment session. Home exercises are introduced consecutively; recommendations included at least three exercises, to perform twice per week over one year.

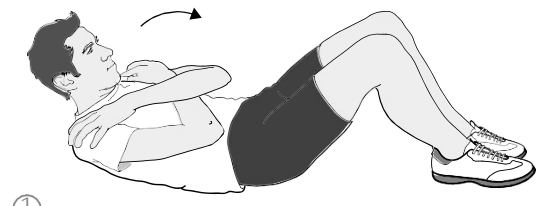

(1)

Rectus abdominis and oblique abdominal muscle training with half sit ups. All directions are allowed. Progression with lifting and extension of legs.

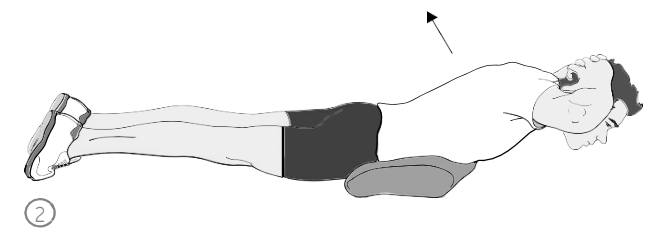

Back extensor training. The lumbar spine is supported with pillows. Progression possible with straight arms and weights.

with ifting and extension of legs.
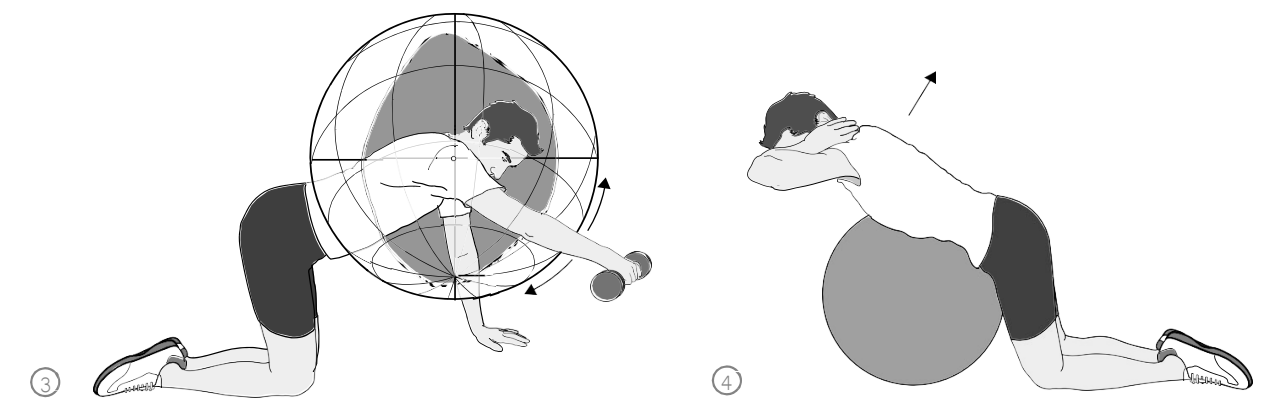

Back extensor training in four-point Back extensor training with Swiss Ball. kneeling. Initially patients lift arms and/or Lumbar spine is supported at all times. legs solely, progression is with weights in Levers of arm can be changed according all directions. to the patients' abilities 

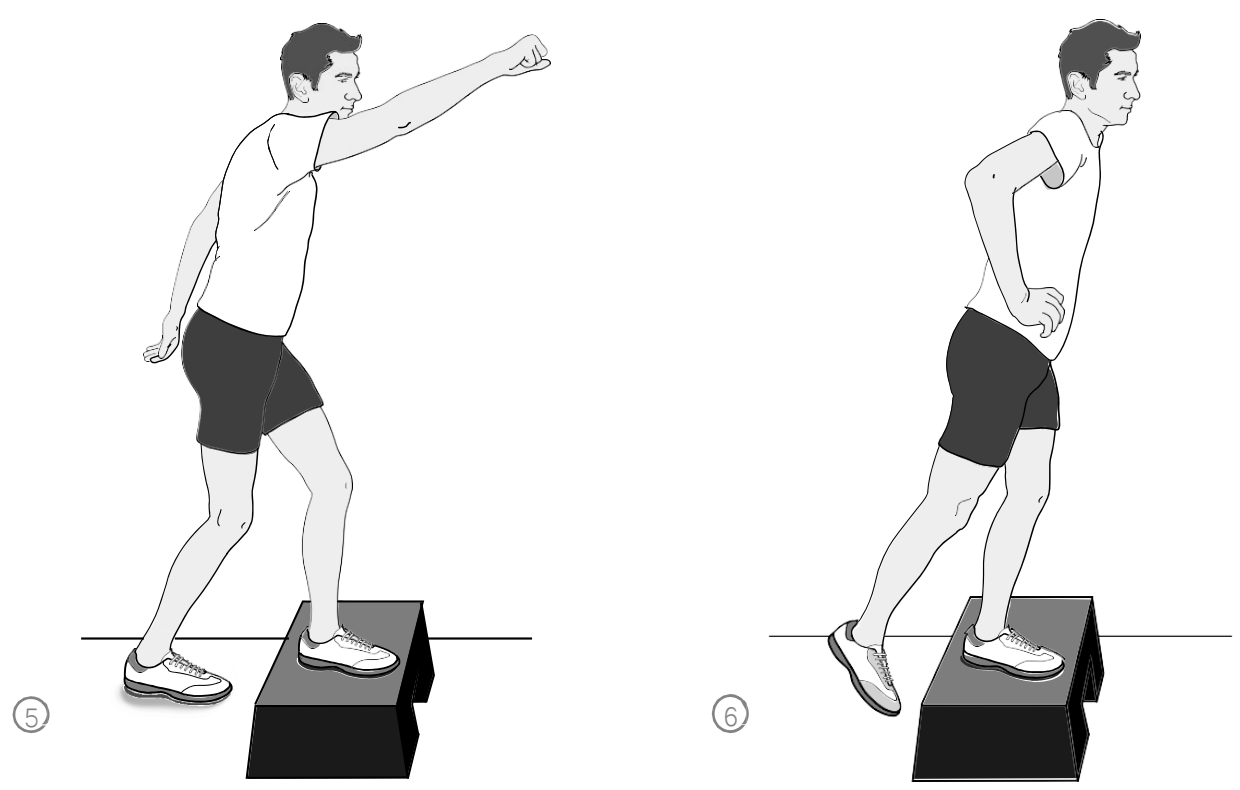

Leg muscles are generally exercised by step ups and downs in varying dosage according to the abilities of the patient. Progression of step exercises by keeping load on one leg during flexion/extension of knee and hip without stepping off. Can be progressed with weight 

Chapter

\begin{abstract}
A tailored exercise program versus general exercise for a subgroup of patients with low back pain and movement control impairment: a randomised controlled trial with one-year follow-up
\end{abstract}




\section{Abstract}

Background: Exercise is an effective treatment for patients with sub-acute and chronic non-specific low back pain (NSLBP). Previous studies have shown that a subgroup of patients with NSLBP and movement control impairment $(\mathrm{MCl})$ can be diagnosed with substantial reliability. However, which type of exercises are most beneficial to this subgroup is still unknown.

Objectives: The effectiveness of a specific exercise treatment to improve movement control was tested in this study.

Methods: Using a multicentre randomised controlled trial (RCT), we compared exercises that targeted $\mathrm{MCl}(\mathrm{MC})$ with a general exercise (GE) treatment. After randomisation, patients in both groups $\mathrm{n}(\mathrm{MC}=52 ; \mathrm{GE}=54)$ were treated in eight private physiotherapy practices and five hospital outpatient physiotherapy centres. Follow-up measurements were taken at post-treatment, six months and 12 months. The primary outcome measurement was the Patient Specific Function Scale (PSFS).

Results: PSFS showed no difference between groups after treatment, or at six months and 12 months. Secondary outcome analysis for pain and disability, measured with the Graded Chronic Pain scale and the Roland Morris Disability Questionnaire respectively, showed that a small improvement post-treatment levelled off over the long term. Both groups improved significantly $(p<0.001)$ over the course of one year.

Conclusion: This study found no additional benefit of specific exercises targeting $\mathrm{MCl}$.

Keywords: randomised controlled trial, movement control impairment, exercise, low back pain, clinical trial, disability 


\section{Introduction}

Sixty to eighty percent of the adult population suffers from low back pain (LBP) at some point during life (Airaksinen et al. , 2006). A previous episode of back pain is highly predictive of future episodes (Kolb et al. , 2011, Stanton et al. , 2008). In most cases, according to guidelines, a specific diagnosis is not possible and the complaints are labelled as non-specific low back pain (NSLBP) (Airaksinen, Brox, 2006, Waddell, 1987).

Evidence shows that exercise in general is an effective treatment for patients with sub-acute or chronic NSLBP (Hayden et al. , 2005). Due to the great heterogeneity of this patient group, clinicians and researchers have tried to identify subgroups of NSLBP that respond positively to and benefit most from a specific exercise treatment (Foster et al. , 2011, Karayannis et al. , 2012). One potential subgroup are patients with movement control impairment $(\mathrm{MCl})$, as classified by O'Sullivan (O'Sullivan, 2005). Patients with $\mathrm{MCl}$ present with mechanically induced pain in static postures together with visible movement abnormalities, such as decreased or increased movement of parts of the lumbar spine, or discrepancies in the proportion of hip, leg and spine movements. It is assumed that these movement abnormalities are influenced by current pain, previous pain episodes and the belief that pain provoked by movement is harmful (O'Sullivan, 2005). The classification of $\mathrm{MCl}$ is based on the observation of aberrant movements accompanied by postural pain (O'Sullivan, 2005). A further sub-classification of $\mathrm{MCl}$ identifies the specific movement direction in which control is reduced (Dankaerts and O'Sullivan, 2011, Dankaerts et al. , 2006). The sub-classification categories are flexion, active extension, passive extension, lateral shift or multidirectional $\mathrm{MCl}$ (O'Sullivan, 2005). Many MCl test procedures have been developed in recent years, (Carlsson and Rasmussen-Barr, 2013, O'Sullivan, 2005, Sahrmann, 2002). In order to define MCl subgroups more clearly, several tests have been evaluated by Luomajoki with a set of $6 \mathrm{MCl}$ tests showing substantial intra-rater and inter-rater reliability (Luomajoki et al. , 2007). $\mathrm{MCl}$ tests were shown to effectively distinguish healthy persons from patients with LBP (Luomajoki et al. , 2008, 2010).

On the assumption that $\mathrm{MCl}$ patients would show better outcomes from treatment targeted at the individual $\mathrm{MCl}$ sub-classification, specific exercises were developed that aimed at relearning normal movement patterns (Luomajoki, Kool, 2010). These exercises were performed with increasing levels of difficulty (Luomajoki, 2010). Patients performed initial, easy, low load exercises, e.g. the positioning of the spine in a neutral position, and progressed to increased load and more complex functional requirements. Finally, unconscious application of the learnt movement patterns in daily activities was trained. These $\mathrm{MCl}$ subgroup specific exercises were targeted at the functional movement problems of the individual patient. However, it remains unclear as to whether individually-tailored treatment leads to superior outcomes.

To date, the proposed mechanisms and treatment of local stabilising muscles, such as multifidi and transversus abdominis, have received considerable attention in spinal 
control research. Two recent meta-analyses measuring pain and disability outcomes compared specific motor control exercises with other forms of exercises but obtained different results. Both papers showed favourable outcomes on both pain and disability for motor control over other forms of exercise in the short and intermediate term. They also agreed that no long term benefit on pain was seen. However, there was disagreement regarding the long term effect on disability. (Bystrom et al. , 2013, Smith et al. , 2014). An $\mathrm{RCT}$ assessing the effectiveness of exercises and behavioural treatment for $\mathrm{MCl}$, as proposed by O'Sullivan, showed some evidence of improved disability and pain when compared with manual therapy and exercise (Vibe Fersum et al. , 2013). However, the latter study is regarded of moderate quality due to the substantial loss of patients in follow-up and to a lack of intention to treat analysis. Furthermore, the question remains unanswered as to which was responsible for the difference: the exercises or the behavioural approach.

To clarify, which exercise approach is superior for patients in the $\mathrm{MCl}$ subgroups, movement control ( $\mathrm{MC}$ ) exercises were compared with general exercises (GE) in this current study. A clearly described general exercise programme was selected for the control group to allow for a realistic treatment option. A previous study, in which patients were not assessed for subgroups, had found a better short term effect on disability in patients with LBP than lumbar stabilising exercise plus general exercise (Koumantakis et al. , 2005).

We studied the effects of specific movement control exercises versus general exercise in a multicentre RCT. This article reports on the results at the six-month and 12 months follow-up and demonstrates the effect on disability and pain of specifically-tailored, active exercise treatment compared with general exercise treatment in patients with NSLBP and $\mathrm{MCl}$.

\section{Methods and material}

\section{Trial Design}

A parallel-group RCT with follow-ups at six months and 12 months was performed in five hospital outpatient departments and eight private practices in Switzerland. Patients were recruited from referring hospitals and resident physicians, as well as through advertising amongst the staff and students of the Zurich University of Applied Sciences, Winterthur, Switzerland (ZHAW). The trial was registered (ISRCTN80064281) and ethical approval obtained from the Swiss Ethics Committee KEK-ZH-NR: 2010-0034/5. The protocol has been previously published (Saner et al. , 2011).

\section{Participants}

Patients presenting with sub-acute or chronic low back pain (persisting for longer than six weeks and no radiating symptoms below the knee) were recruited. Age had to be 18 
to 75 years. Eligible patients also had to present with: predefined $\mathrm{MCl}$ complaints (pain provocation in static positions) (Dankaerts and O'Sullivan, 2011), together with a score of two or more positive results out of the six MCl tests (Luomajoki, Kool, 2007). Disability levels had to be at least five points on the Roland Morris Disability Questionnaire (RMDQ) (Pengel et al. , 2004, Roland and Morris, 1983, Wiesinger et al. , 1999).

Excluded were patients (1) with LBP due to known or suspected specific causes, with recent surgery on the spine (< 6 weeks); (2) with spondylodesis; (3) with comorbid health conditions, which limited exercise training. (4) To focus on patients with pain responding to movement, we also excluded patients complaining of constant pain and/or pain below the knee. (5) To avoid confounding by psychosocial factors, patients with a score of more than 130 on the Örebrö Musculoskeletal Pain Screening Questionnaire (ÖMPSQ) (Linton and Boersma, 2003) and/or (6) more than 3 months of sick leave due to LBP were also excluded. German language had to be sufficient to understand study information, instructions and questionnaires. For further details see flow chart Figure 1.

\section{Randomisation and blinding}

Following signing informed consent, patients attended baseline assessment, which was conducted by an independent, experienced and specially-trained physiotherapist. Eligible patients were randomly allocated to one of two groups. The computer-generated randomisation schedule was produced before trial start, using block-randomisation with a block size of four. Group allocation was communicated to the therapist by means of telephone contact from an independent research assistant at the ZHAW. Outcome assessors and data analysts were blinded to the allocation and were not involved in treatment throughout the trial. Blinding of patients and physiotherapists to the allocation was not possible, but patients were kept naïve to the specific research goal. Physiotherapists of the $\mathrm{MC}$ group received all baseline information concerning the $\mathrm{MCl}$ sub-classification and instruction on the six physical tests, in order to plan and apply a specific treatment. All other physiotherapists involved remained masked for initial assessments and trial results. 


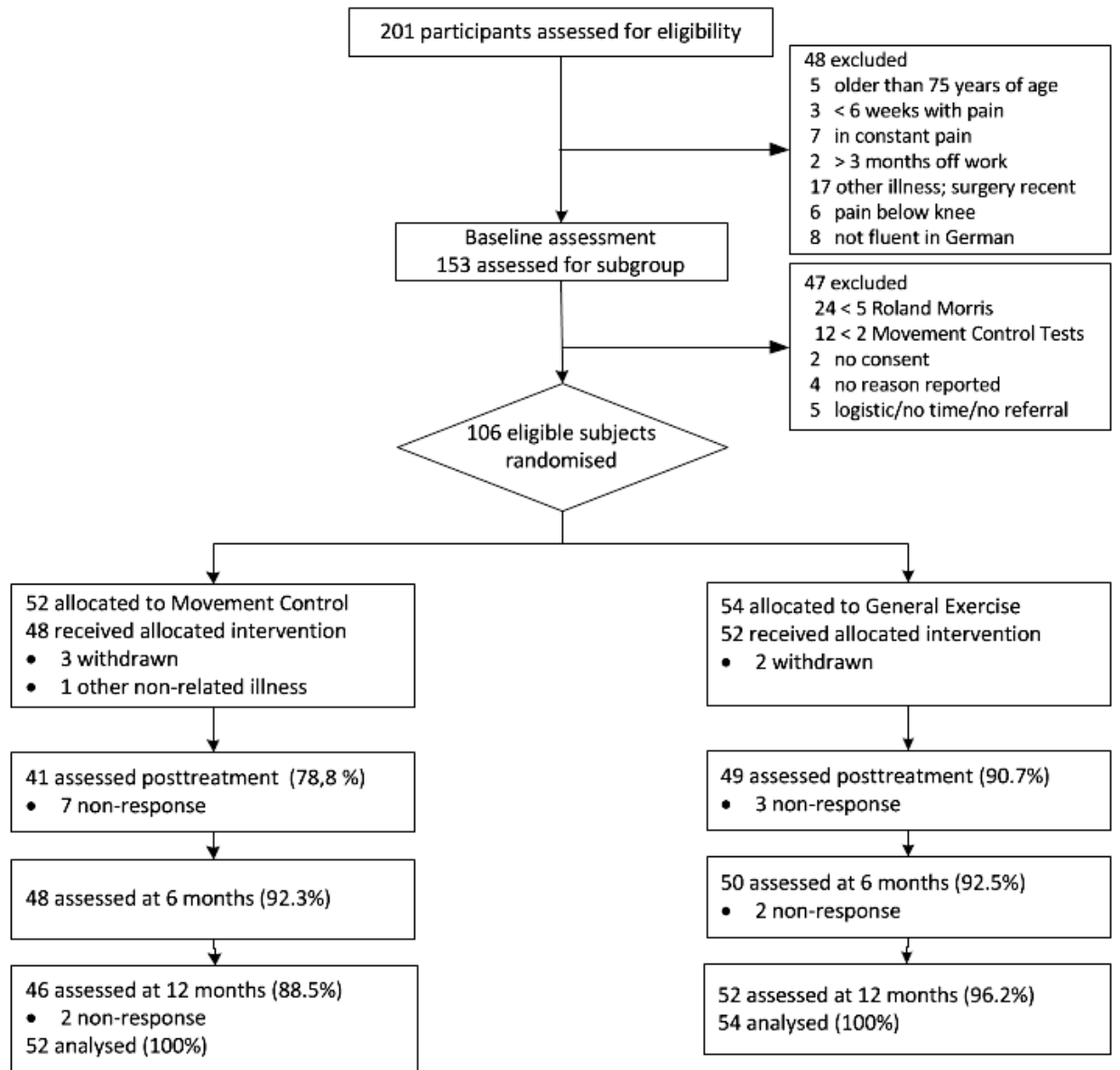

Figure 1 Flowchart of participants through the trial.

Non-response rate is only valid for the equivalent follow up.

\section{Interventions}

Patients in both groups received individual treatment sessions of 30 minutes, preferably twice per week, over a period of nine to 12 weeks. Progression of the treatment, in accordance with the treatment protocol, was determined by the physiotherapist. Ten minutes of each session was allowed for other physiotherapy applications, where necessary. The length and type of additional interventions were recorded and monitored. All patients received instructions for a minimum of three home exercises and were encouraged to practise them at least twice a week for up to one year after treatment. Patients were contacted by telephone after six months and encouraged to maintain the training.

Movement control (MC) treatment consisted of active exercises addressing the pain-provoking postures and control of the impaired movement(s). These were assessed and 
classified at baseline, as proposed by O'Sullivan and Luomajoki (Dankaerts, O'Sullivan, 2006, O'Sullivan, 2005) (Luomajoki, Kool, 2010). As initial exercises, patients learned to perform controlled movements with low load in supported positions. Later they progressed to open chain positions, to exercises involving controlled movements with increased load and to specific functional tasks. Strength and endurance training was allowed once movement control was achieved. Following the rationale that the $\mathrm{MC}$ test battery is representative of functional $\mathrm{MCl}$, the focus of the treatment was on functional restoration of the impaired movement(s). Exercises aimed specifically at local lumbar stabilising muscles (Hides et al. , 2001), or treatment according to behavioural classification as recently proposed by O'Sullivan (O'Sullivan, 2012), were not included in the MCl treatment protocol. For example, patient $A$, when asked to bend forward without moving the lower part of the spine, was not able to stabilise the lumbar spine: this $\mathrm{MCl}$ was classified as flexion impairment, and the corresponding test is called "waiter's bow". In treatment, the patient learns to control the lumbar spine stepwise: initially performing exercises with hip movements only in well-supported body positions (such as lying and sitting) and gradually progressing to exercises in standing. Load, frequency and velocity can gradually be increased once the movement is retrained. The ultimate goal is that patient A should be able to move freely and automatically in complex functional situations according to his personal needs in daily life.

General exercise (GE) treatment aimed to improve the muscular strength of the lumbar and pelvic region and legs. In a standardised programme, as described in a study manual, all relevant muscle groups (abdominals, erector spinae, gluteals, quadriceps and hamstrings) were addressed in each treatment (Koumantakis, Watson, 2005). Start load and progression were assessed individually and followed a submaximal training protocol, according to the guidelines of the American College of Sports Medicine (Whaley, 2006).

The main contrast between the two programmes was the tailored-exercise training targeting functional improvement of $\mathrm{MCl}$ in the experimental group, as opposed to the non-specific general strength training performed by the control group.

All therapists showed a positive attitude towards their treatment group and had used the treatments in their daily practice. Therapists in the MC group were either trained to OMT (Orthopaedic Manual Therapy) standard or were novice physiotherapists working under the supervision of a highly-qualified OMT clinician of the ZHAW. All physiotherapists attended at least four hours of specific training on the protocol of their respective treatment. Additionally, they received a manual of conduct and exercise procedures. The therapists reported the trained exercises, number and description of home exercises and other interventions for each patient in a written log. 


\section{Baseline descriptives}

Demographic and psychosocial characteristics of age, sex, height, weight, sports activities, work load, work status, sickness abscence, medication, duration of LBP-related symptoms and Örebrö musculoskeletal pain questionnaire (ÖMPQ) were recorded at baseline.

Table 2 Baseline demographic data and baseline results of questionnaires

\begin{tabular}{|c|c|c|c|c|}
\hline & & $\begin{array}{l}\text { Movement Control } \\
n=52\end{array}$ & $\begin{array}{l}\text { General Exercise } \\
n=54\end{array}$ & $\begin{array}{l}\text { Total Group } \\
n=106\end{array}$ \\
\hline Age (y) & mean (SD) & $42.8(13.8)$ & $40.5(14.7)$ & $41.6(14.1)$ \\
\hline Gender & female, $n$ & 16 & 24 & 40 \\
\hline Height (cm) & mean (SD) & $173.0(8.5)$ & $173.9(8.0)$ & $173.6(8.3)$ \\
\hline Weight (kg) & mean (SD) & $75.5(11.7)$ & 74.7 (11.9) & $75.4(12.1)$ \\
\hline \multirow[t]{4}{*}{ Physical workload } & low, $n$ & $23(44.2 \%)$ & $21(38.9 \%)$ & $44(41.5 \%)$ \\
\hline & medium, $\mathrm{n}$ & $22(42.3 \%)$ & $26(48.1 \%)$ & $48(45.3 \%)$ \\
\hline & heavy, n & $5(9.6 \%)$ & $6(11.1 \%)$ & 11 (10.4\%) \\
\hline & missing, $n$ & $2(3.8 \%)$ & $1(1.9 \%)$ & $3(2.8 \%)$ \\
\hline \multirow[t]{4}{*}{ Sport participation } & no & $19(36.5 \%)$ & $16(30.8 \%)$ & 35 (33.0\%) \\
\hline & $1-2 x$ week & $21(42.0 \%)$ & 23 (43.1\%) & 44 (41.5\%) \\
\hline & $>2 x$ week & $10(20.0 \%)$ & 13 (25.0\%) & 23 (21.7\%) \\
\hline & missing & $2(3.8 \%)$ & $2(3.8 \%)$ & $4(3.8 \%)$ \\
\hline \multirow[t]{5}{*}{ Work status, $\mathrm{n}$} & sick leave & $1(1.9 \%)$ & $5(9.3 \%)$ & $6(5.7 \%)$ \\
\hline & part-time leave & $3(5.8 \%)$ & $3(5.6 \%)$ & $6(5.7 \%)$ \\
\hline & full-time & 39 (75.0\%) & $38(70.4 \%)$ & 77 (72.6\%) \\
\hline & no paid job & $5(9.6 \%)$ & $8(14.8 \%)$ & 13 (12.3\%) \\
\hline & missing & $4(7.7 \%)$ & 0 & $4(3.8 \%)$ \\
\hline \multirow[t]{5}{*}{ Pain Duration* $(y)$} & mean (SD) & $11.6(12.8)$ & $8.4(8.9)$ & 10.0 (11.0) \\
\hline & $<1$ year, $\mathrm{n}$ & 4 & 8 & 12 (11.3\%) \\
\hline & $1-5$ years, $n$ & 17 & 15 & 32 (30.2\%) \\
\hline & $>5$ years, $n$ & 26 & 27 & 53 (50.0\%) \\
\hline & Missing, $n$ & 5 & 4 & $9(8.5 \%)$ \\
\hline ÖMPQ & mean (SD) & $78.3(24.3)$ & $81.5(20.4)$ & $79.9(22.3)$ \\
\hline
\end{tabular}

Abbreviations: $y$ = year; SD = standard deviation; ÖMPQ: Oerebrö musculoskeletal pain questionnaire. * Overall duration of LBP

\section{Outcome measures}

Measurements of outcome were taken at baseline, post-treatment, after six months and 12 months. Outcome measures pre- and post-treatment were obtained by a research assistant onsite. Research assistants at the study centre collected primary outcome measures at six months and 12 months by telephone. On this occasion, they communicated the questionnaire on secondary outcomes, which were delivered and returned by postal mail.

Primary outcome was patient-specific LBP-related activity limitation measured with the Patient Specific Functional Scale (PSFS), the latter having shown excellent reliability for mechanical and chronic LBP (Hall et al. , 2011, Horn et al. , 2012, Stratford P, 1995) 
and concurrent validity with Roland-Morris Disability Questionnaire (RMDQ). PSFS was selected because it showed good responsiveness in patients with moderate LBP complaints and reflected patients' individual relevant limitations (Hall et al. , 2010, Pengel, Refshauge, 2004).

Secondary outcome variables were pain over the last three months (measured with the subscales "Characteristic Pain Intensity" of the Graded Chronic Pain Scale (GCPS) (Turk, 2011) (v. 2.0)) and disability (measured with the subscales "Disability Score" of the GCPS and the Roland-Morris Disability Questionnaire (RMDQ) (Roland and Morris, 1983)). Total GCPS scores for pain ranged from 0-30 and for disability from 0-40. Reliability and validity of GCPS and RMDQ were high for the English and the German versions

(Klasen et al. , 2004, Roland and Fairbank, 2000, Wiesinger, Nuhr, 1999). More details about outcome measurements are described in the study protocol (Saner, Kool, 2011).

\section{Adherence and satisfaction}

Adherence to treatment by patients and therapists was monitored using log books and a comprehensive questionnaire, both during and after treatment. Patient satisfaction was assessed with a numeric rating scale from 0 (extremely dissatisfied) to 10 (completely satisfied) after six months and one year.

\section{Data analysis}

Descriptive statistics of demographic and clinical measures were performed. Analyses followed the intention-to-treat principle. For the primary outcome, only the first activity mentioned in the PSFS was used for analysis, i.e. the first open-ended response item, since correlation between the averages of the three activities versus the first activity was very high at 0.9. Univariate analysis of variance (ANOVA) for the primary outcome and subsequent analysis of covariance (ANCOVA) analysed the potential influence of the identified covariates of baseline differences and pain duration. Missing data for this analysis were replaced by the group mean value (Hollis and Campbell, 1999).

For primary and secondary outcome, we fitted a linear mixed model (LMM) to the data with time, treatment group and the interaction time: treatment group as fixed effects, subject was included as random intercept (Son et al. , 2012). Random intercept models are equivalent to repeated measures ANOVA and take into account the correlation between repeated measurements. In contrast to classical repeated measures ANOVA, they can deal naturally with missing observations. All missing values were handled in the model as missing at random. In a first step, the parameters of the model were estimated; in a second step, specific contrasts were estimated.

For the intervention, we described differences of adherence between both groups regarding frequency of home exercises and patient satisfaction. For work status descriptive data over one year were provided. 
Analyses were conducted using IBM SPSS Statistics 20. Two-sided significance was set at $p \leq 0.05$.

\section{Results}

\section{Participants}

Between August 2010 and February 2012 a total of 201 patients were evaluated for eligibility. As described in the flow chart (Figure 1), 48 patients did not meet the primary inclusion criteria. A further 47 patients were excluded from randomisation after baseline assessment. The main reasons for exclusion were minimal disability ( $<5 \mathrm{RMDQ}$ ) or no movement control impairment ( $<2 \mathrm{MCl}$ tests positive). After signing informed consent, 106 patients were randomised $(\mathrm{MC}=52, \mathrm{GE}=54)$. The final number of participants equates to the sample size calculation (power 0.9; 2-sided a 0.05; 10\% drop out)(Saner, Kool, 2011). Follow-up data collection ended in March 2013.

Table 1 shows that most baseline characteristics were similar in both groups. There were more men than women, participants were relatively active in sports, only $11 \%$ were absent from work or on restricted work because of their LBP. Participants with LBP for longer than one year comprised $80 \%$. Patients in the MC group had experienced a longer mean duration of pain than those in the GE group.

For unknown reasons, three patients in the $M C$ and two in the GE group withdrew from the study during treatment. One patient withdrew for other unrelated medical reasons. Baseline characteristics did not differ between assessed and non-assessed patients during follow-up, except for the duration of pain (MC/GE;9.0/15.7 years). The attrition rate of six $\%$ was within the anticipated $10 \%$.

\section{Primary outcome}

Table 2 and Figure 2 show outcomes for LMM of treatment effects of PSFS at all followups. Both groups improved significantly over time $(p<0.001)$. The time and group interaction effect for the PSFS was not significant ( $p>0.05$ ). A slight post-treatment trend in favour of the MC group (mean $-0.4 ;-1.4-0.6,95 \% \mathrm{Cl}$ ) levelled off at 6 months. Baseline differences and information from the literature identified pain duration as potentially having an influence on outcomes (Dunn and Croft, 2006, Hill et al. , 2008, Von Korff and Dunn, 2008). However, the results were not changed to a level of significance and these variables were not taken into account for further analysis (LMM)(Ryoo, 2011).

The minimal clinical important change was reported $>0.9$ on PSFS for mechanical pain (Stratford P, 1995)and >1.9 for chronic pain (Maughan and Lewis, 2010). 95 patients (89.9\%) reached the first (MC/GE;90.4\%/88.9\%) and 81 (76.4\%) the second value (MC/GE;75.0\%/77.8\%) 


\section{Secondary outcomes}

Figure 2 and Table 2 show the results of GCPS and RMDQ. Pain differences were nonsignificant at all follow-ups. The between-group difference for the RMDQ in favour of the $\mathrm{MCl}$ group, which was significant post-treatment, was no longer significant after six and 12 months.

As in the primary outcome, all secondary outcomes improved significantly over time in both groups. Again, the main effect over time was shown between pre- and post-treatment and improvements were maintained for up to one year.

All patients, who were initially on full or partial sick leave because of their back pain, improved their work status. One patient in the GE group remained on partial sick leave.
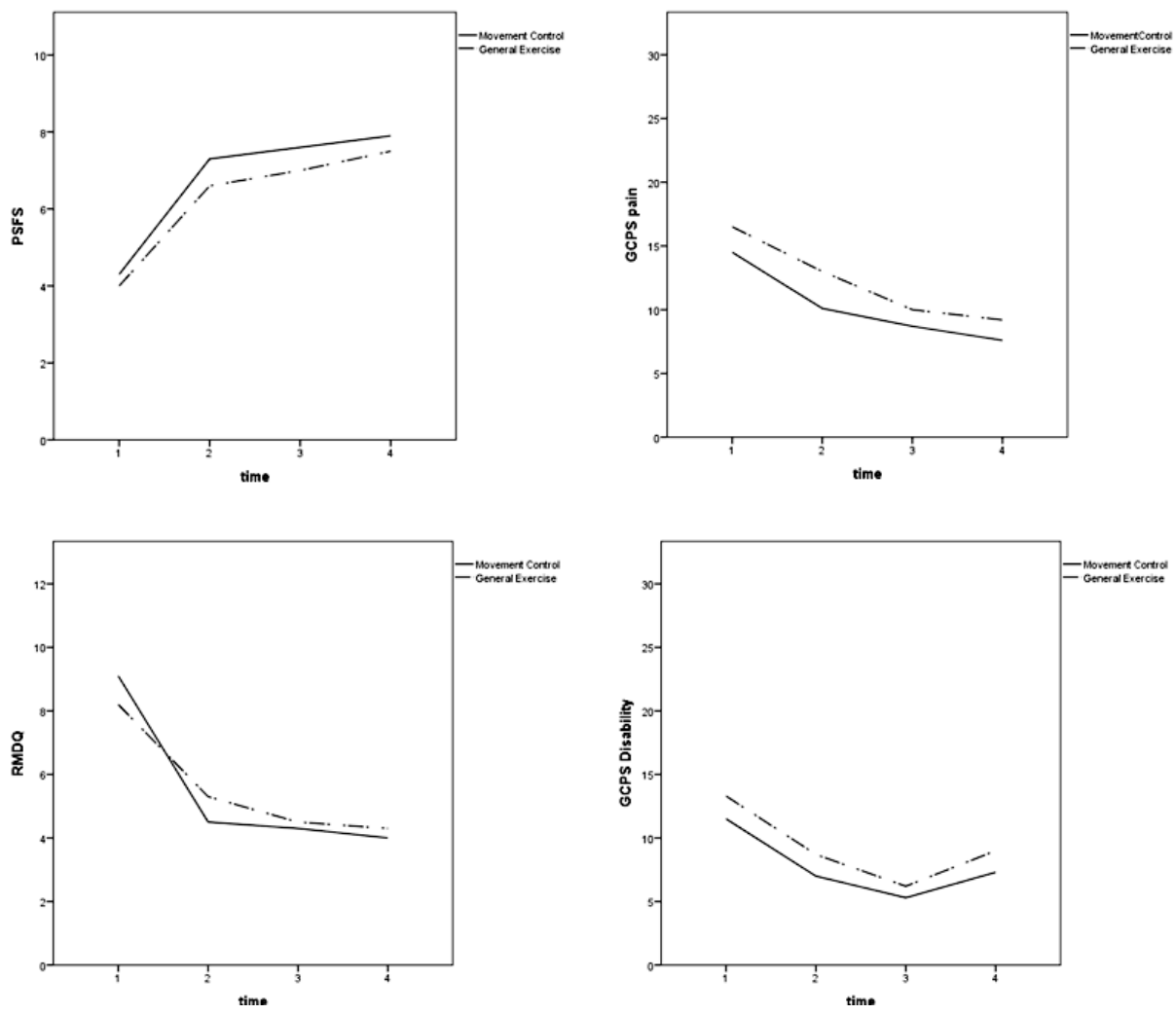

Figure 2: Long term results of primary and secondary outcomes.

Patient Specific Functional Scale (PSFS) (0-10); Graded Chronic Pain Scale (GCPS) subscale for characteristic pain (0-30); Roland Morris Disability Scale (RMDQ) (0-24); GCPS subscale disability (0-40); measured at baseline (time1), post-treatment (time2), after 6 months (time3) and 12 months (time4). 
Table 2: Outcomes of linear mixed model (LMM) for primary and for secondary results of each group. Treatment effects measured post-treatment, 6 months and 12 months.

\begin{tabular}{|c|c|c|c|c|}
\hline \multicolumn{5}{|l|}{ Primary outcome } \\
\hline & $\begin{array}{l}\text { Movement Control } \\
n=52\end{array}$ & $\begin{array}{l}\text { General Exercise } \\
\mathrm{n}=54\end{array}$ & $\begin{array}{l}\text { Estimated Mean difference } \\
(95 \% \mathrm{Cl})\end{array}$ & $p$ \\
\hline \multicolumn{5}{|c|}{ Patient Specific Functional Scale (PSFS) } \\
\hline \multicolumn{5}{|c|}{ Overall time $x$ group interaction effect: $F=0.2 p=0.89$} \\
\hline Baseline & 4.3 & 4 & & \\
\hline Post-treatment & 7.3 & 6.6 & $-0.4(-1.4-0.6)$ & 0.48 \\
\hline 6 months & 7.6 & 7.0 & $-0.2(-1.3-0.7)$ & 0.62 \\
\hline 12 months & 7.9 & 7.5 & $-0.08(-1.1-0.9)$ & 0.88 \\
\hline \multicolumn{5}{|c|}{ Secondary outcome } \\
\hline \multicolumn{5}{|c|}{ Graded Chronic Pain Scale - Characteristic Pain Intensity in last 3 months (GCPS sub-score pain) } \\
\hline \multicolumn{5}{|c|}{ Overall time $x$ group interaction effect: $F=0.6 p=0.6$} \\
\hline Baseline & 14.5 & 16.5 & & \\
\hline Post-treatment & 10.1 & 13.0 & $0.8(-1.4-3.1)$ & 0.47 \\
\hline 6 months & 8.7 & 10.0 & $-0.7(-3.0-1.8)$ & 0.55 \\
\hline 12 months & 7.6 & 9.2 & $-0.5(-2.8-1.8)$ & 0.69 \\
\hline \multicolumn{5}{|c|}{ Roland Morris Disability Questionnaire (RMDQ) } \\
\hline \multicolumn{5}{|c|}{ Overall time $x$ group interaction effect: $F=1.6 p=0.19$} \\
\hline Baseline & 9.1 & 8.2 & & \\
\hline Post-treatment & 4.5 & 5.3 & $1.6(0.1-3.1)$ & $0.04^{*}$ \\
\hline 6 months & 4.3 & 4.5 & $1.0(-0.5-2.5)$ & 0.19 \\
\hline 12 months & 4.0 & 4.3 & $1.6(-0.4-2.6)$ & 0.13 \\
\hline \multicolumn{5}{|c|}{ Graded Chronic Pain Scale - Disability in last 3 months (GCPS sub-score disability) } \\
\hline \multicolumn{5}{|c|}{ Overall time $x$ group interaction effect: $F=0.05 p=0.9$} \\
\hline Baseline & 11.5 & 13.3 & & \\
\hline Post-treatment & 7.0 & 8.7 & $-0.2(-6.1-5.8)$ & 0.9 \\
\hline 6 months & 5.3 & 6.2 & $-1.1(-7.1-5.0)$ & 0.7 \\
\hline 12 months & 7.3 & 9.0 & $-0.2(-6.2-5.8)$ & 0.9 \\
\hline \multicolumn{5}{|c|}{ Graded Chronic Pain Scale (GCPS total score) } \\
\hline \multicolumn{5}{|c|}{ Overall time $x$ group interaction effect: $F=0.6 p=0.6$} \\
\hline Baseline & 25.9 & 29.5 & & \\
\hline Post-treatment & 17.5 & 21.8 & $0.4(-4.2-5.1)$ & 0.8 \\
\hline 6 months & 14.2 & 16.6 & $-1.5(-6.3-3.3)$ & 0.5 \\
\hline 12 months & 12.4 & 14.4 & $-2.4(-7.1-2.3)$ & 0.3 \\
\hline
\end{tabular}

For PSFS self-score activity mentioned first from 0 (unable to perform) to 10 (able to perform without difficulties).

RMDQ score on 24 items on a 0 (able to perform without difficulties) to 1 (perform with difficulties) scale.

GCPS characteristic pain intensity on three items (actual pain, worst pain, usual pain).

GCPS disability score on four items (days kept from activities, inference with daily, social and work activities) both on a 0 (for no pain) to 10 (for pain as bad as could be) scale.

GCPS total score 0 to 70 adding sub-score for pain and disability.

Overall effect shows results over 12 months. Data are unadjusted mean for each time point. Estimated Mean difference $(95 \% \mathrm{Cl})$ contains results between each time period. Minus scores of mean difference in PSFS favour $\mathrm{MC}$, in all secondary outcomes minus scores favour GE. 


\section{Treatment descriptives}

Patients received a mean of $8-9$ treatment sessions (range MC 4-18; GE 5-25) in both groups. Patients completing treatment reported a mean number of recommended exercises of MC/GE 3.9/5.0, respectively. One year after randomisation, approximately $46 \%$ of the patients in both groups (MC/GE; $=20 / 22$ ) reported that they still did their exercises in accordance with the recommended twice per week or daily. Patient satisfaction with treatment was comparable between groups. At the six months and twelve months followups $70 \%$ and $80 \%$ of the patients in both groups rated satisfaction with treatment as high to very high (8 or more on a 0 - 10 scale)(Kool et al. , 2007).

Patients in both groups started with a mean number of impaired movement control tests of 3.9, the MC group improved to a mean of 1.8 and the GE patients to 2.8 positive tests after treatment. The 10 minutes allocated to other physiotherapy applications was monitored in the log book and did not exceed the allowance for any patient.

A total of 28 physiotherapists performed the treatments. Therapists in both groups had mixed levels of experience. Due to the two different recruitment processes, therapists in private practice reported mean years of experience MC/GE; 12.9/9.8. Physiotherapists from the ZHAW (four therapists in each group) were novices and were treating 15 patients in each group.

\section{Discussion}

The findings of this study indicate no additional benefit on disability and pain to patients with NSLBP and $\mathrm{MCl}$ of movement control exercise versus general exercise. Both groups improved significantly on all outcomes over time.

\section{Strengths of this study}

This trial was prospectively registered, the protocol was published and every attempt was made in the design to minimise bias.

In general, patients were representative of people with mild to moderate pain and disability as a result of their long-lasting low back disorders. Patients with a high risk of psychosocial burden were excluded, in order to principally address the physical aspects of NSLBP.

For the interventions, we chose two exercise programmes, which had previously shown positive effects, with the aim of clarifying the choice of treatment for the physiotherapist. The pragmatic approach, with 28 physiotherapists in different clinical settings and therapists with different length of work experience, shows that the treatments are widely applicable.

The adherence to the intervention protocol was high for therapists and patients in both groups. A blinded research assistant, who obtained the third and fourth PSFS results 
by telephone, also encouraged participants to stay compliant with their home exercise programme. This might have supported the high adherence and follow-up rates (Evers et al. , 2012).

\section{Limitations of this study}

The unexpected improvements in both groups may be influenced by the inclusion criteria of the study. Previous observational studies, analysing the natural history of LBP, have stated repeatedly that patients with previous episodes of back pain are likely to remain in a stable situation over years with no improvement over time (Tamcan et al. , 2010, Von Korff, 1994). In our RCT, a selection bias towards a NSLBP population with low psychosocial influence and high self-management competence may be present and must be considered when selecting either exercise programme for future treatment. Additionally, the inclusion criteria of $\mathrm{MCl}-2$ positive tests out of 6 - was designed to select patients who would have the best chance of improvement with specific exercises. Likewise, it was not possible to blind physiotherapists or patients due to the nature of the treatment; patients would have noticed during treatment whether they were performing a general exercise program or tailored, focused exercises that matched the tests they underwent at baseline. The therapists treating patients in the $\mathrm{MCl}$ group had an OMT degree, which is a two-year postgraduate specialisation in musculoskeletal physiotherapy. It was assumed that they were sufficiently specialised in treating $\mathrm{MCl}$. However, O'Sullivan claims that it needs a further 100-hour programme to learn to treat patients in this subgroup. Therefore, the equal results might be due to insufficient training. However, in our opinion, treatment of $\mathrm{MCl}$ is achievable by every physiotherapist, should be not too complex and form part of every physiotherapists toolbox.

\section{Comparison with other studies}

We are not aware of any results from other RCTs with the same inclusion criteria and this type of MC treatment. In a study of moderate methodological quality, a similar patient group, identified with the same classification system, found a significant benefit from behaviourally-orientated exercises when compared with spinal manipulation plus exercise (Vibe Fersum, O'Sullivan, 2013). Other than in our study, patients were additionally classified according to their behavioural pattern. The intervention of the experimental group followed a mixed approach of exercise and cognitive behavioural treatment. We are aware of changes to the assessment and treatment approaches in more recent years (O'Sullivan, 2012). Nowadays, non-specific low back pain disorders should be considered within the multidimensional bio-psycho-social framework. Implementation of this approach is of major concern regarding the "beliefs" of the therapists in terms of how they understand and deal with NSLBP. 


\section{The importance of exercise in NSLBP}

The results of our study support previous findings that exercise in general, regardless of the type, is beneficial for patients with NSLBP. Alongside disability, pain, a major concern for patients, improved significantly and continuously. Clearly both treatments share general effective aspects of exercise. All patients receive attention to their complaints, they are introduced to exercise at their level of fitness, and the exercises are controlled regularly. As a result, they improve their physical activity levels, fitness and gain confidence in movement and adjustment of lifestyle.

Furthermore, the importance of general influences of the therapeutic relationship and the effects on pain beliefs, as explained in the common factors model, may be underestimated (Hall, Ferreira, 2010, Miciak et al. , 2012). This assumption is supported by the high level of satisfaction with treatment in both groups. Although all these factors are likely to explain a substantial part of the improvement in both groups, the previously mentioned effect of some degree of selection bias towards a sample with an existing good prognosis, cannot be ruled out.

\section{Conclusion}

Contrary to our expectation, MC exercise and GE exercise appear equally effective in the patient subgroup included in this study. We can conclude that the contrast between both types of intervention did not bring additional value to the shared effects. Decisions for the application of either active treatment approach can currently not be taken on the basis of the results of this study. It is possible that the type of exercise treatment is less important than previously presumed; that the patient is guided to a consistent long-term exercise lifestyle is of most importance.

Based on the results of this study, we can recommend exercise therapy for patients with NSLBP and $\mathrm{MCl}$, either using movement control or general exercise. Future research on treatment for NSLBP may reconsider the concept of testing exercises for specific subgroups. If the theoretical model, clinical findings, patients likely to respond to the treatment (but with a prognosis that can be improved) and adequate treatment goals are found, the means to treat need to have sufficient contrast. The concept behind exercise may not be based so much on specific movements, but on activity per se, the dosage of exercise, the kind of information and general aspects of a physiotherapy treatment. 


\section{Acknowledgements}

The authors would like to thank all patients who participated in this study.

We wish to thank physiotherapists and doctors in clinics and practices in Switzerland, which are: Bethesda Spital, Basel; Kantonsspital Winterthur, Winterthur; Klinik, Schulthess, Zurich; Medbase Physiotherapie, Winterthur; Physiotherapie Seen, Winterthur; Physiotherapie Bellaria, Zurich; Physiotherapie Erlenbach, Erlenbach; Physiotherapie Reinach, Reinach; Physiotherapie Würenlingen, Würenlingen; Provital Physiotherapie, Egg; Rheumaklinik, Universitätsspital Zurich, Zurich; Segeten Physiotherapie, Zurich; Uniklinik Balgrist, Zurich. We also wish to thank Christa Wachter for her help in the randomisation process, André Meichtry for statistical advice and Karen Linwood-Williams for proof reading the manuscript.

The project was supported by the Swiss National Science Foundation (SNSF).

(Project no. 127240) 


\section{References}

Airaksinen O, Brox J, Cedraschi C, Hildebrandt J, Klaber-Moffett J, Kovacs F, et al. Chapter 4. European guidelines for the management of chronic nonspecific low back pain. Eur Spine J. 2006;15 Suppl 2:S192-300.

Bystrom MG, Rasmussen-Barr E, Grooten WJ. Motor control exercises reduces pain and disability in chronic and recurrent low back pain: a meta-analysis. Spine (Phila Pa 1976). 2013;38:E350-8.

Carlsson H, Rasmussen-Barr E. Clinical screening tests for assessing movement control in non-specific low-back pain. A systematic review of intra- and inter-observer reliability studies. Man Ther. 2013;18:103-10.

Dankaerts W, O'Sullivan P. The validity of O'Sullivan's classification system (CS) for a sub-group of NS-CLBP with motor control impairment $(\mathrm{MCl})$ : overview of a series of studies and review of the literature. Man Ther. 2011;16:9-14.

Dankaerts W, O'Sullivan PB, Straker LM, Burnett AF, Skouen JS. The inter-examiner reliability of a classification method for non-specific chronic low back pain patients with motor control impairment. Manual Therapy. 2006;11:28-39.

Dunn KM, Croft PR. The importance of symptom duration in determining prognosis. Pain. 2006;121:126-32.

Evers A, Klusmann V, Ziegelmann JP, Schwarzer R, Heuser I. Long-term adherence to a physical activity intervention: the role of telephone-assisted vs. self-administered coping plans and strategy use. Psychology \& health. 2012;27:784-97.

Foster NE, Hill JC, Hay EM. Subgrouping patients with low back pain in primary care: are we getting any better at it? Man Ther. 2011;16:3-8.

Hall AM, Ferreira PH, Maher CG, Latimer J, Ferreira ML. The influence of the therapist-patient relationship on treatment outcome in physical rehabilitation: a systematic review. Phys Ther. 2010;90:1099-110.

Hall AM, Maher CG, Latimer J, Ferreira ML, Costa LO. The patient-specific functional scale is more responsive than the Roland Morris disability questionnaire when activity limitation is low. Eur Spine J. 2011;20:79-86.

Hayden JA, van Tulder MW, Malmivaara AV, Koes BW. Meta-analysis: exercise therapy for nonspecific low back pain. Ann Intern Med. 2005;142:765-75.

Hides JA, Jull GA, Richardson CA. Long-term effects of specific stabilizing exercises for first-episode low back pain. Spine (Phila Pa 1976). 2001;26:E243-8.

Hill JC, Dunn KM, Lewis M, Mullis R, Main CJ, Foster NE, et al. A primary care back pain screening tool: identifying patient subgroups for initial treatment. Arthritis Rheum. 2008;59:632-41.

Hollis S, Campbell F. What is meant by intention to treat analysis? Survey of published randomised controlled trials. BMJ. 1999;319:670-4.

Horn KK, Jennings S, Richardson G, Vliet DV, Hefford C, Abbott JH. The patient-specific functional scale: psychometrics, clinimetrics, and application as a clinical outcome measure. J Orthop Sports Phys Ther. 2012;42:30-42.

Karayannis NV, Jull GA, Hodges PW. Physiotherapy movement based classification approaches to low back pain: comparison of subgroups through review and developer/expert survey. BMC Musculoskelet Disord. 2012;13:24

Klasen BW, Hallner D, Schaub C, Willburger R, Hasenbring M. Validation and reliability of the German version of the Chronic Pain Grade questionnaire in primary care back pain patients. Psychosoc Med. 2004;1:Doc07.

Kolb E, Canjuga M, Bauer GF, Laubli T. Course of back pain across 5 years: a retrospective cohort study in the general population of Switzerland. Spine (Phila Pa 1976). 2011;36:E268-73.

Kool J, Bachmann S, Oesch P, Knuesel O, Ambergen T, de Bie R, et al. Function-centered rehabilitation increases work days in patients with nonacute nonspecific low back pain: 1-year results from a randomized controlled trial. Arch Phys Med Rehabil. 2007;88:1089-94.

Koumantakis GA, Watson PJ, Oldham JA. Trunk muscle stabilization training plus general exercise versus general exercise only: randomized controlled trial of patients with recurrent low back pain. Phys Ther. 2005;85:209-25.

Linton SJ, Boersma K. Early identification of patients at risk of developing a persistent back problem: the predictive validity of the Orebro Musculoskeletal Pain Questionnaire. Clin J Pain. 2003;19:80-6. 
Luomajoki H. Movement Control Impairment as a sub-group of non-specific low back pain: Evaluation of movement control test battery as a practical tool in the diagnosis of movement control impairment and treatment of this dysfunction. Kuopio, Finnland: University of Eastern Finland; 2010.

Luomajoki H, Kool J, de Bruin ED, Airaksinen O. Reliability of movement control tests in the lumbar spine. BMC Musculoskelet Disord. 2007;8:90.

Luomajoki H, Kool J, de Bruin ED, Airaksinen O. Movement control tests of the low back; evaluation of the difference between patients with low back pain and healthy controls. BMC Musculoskelet Disord. 2008;9:170.

Luomajoki H, Kool J, de Bruin ED, Airaksinen O. Improvement in low back movement control, decreased pain and disability, resulting from specific exercise intervention. Sports Med Arthrosc Rehabil Ther Technol. 2010;2:11.

Maughan EF, Lewis JS. Outcome measures in chronic low back pain. Eur Spine J. 2010;19:1484-94.

Miciak M, Gross DP, Joyce A. A review of the psychotherapeutic 'common factors' model and its application in physical therapy: the need to consider general effects in physical therapy practice. Scandinavian journal of caring sciences. 2012;26:394-403.

O'Sullivan P. Diagnosis and classification of chronic low back pain disorders: Maladaptive movement and motor control impairments as underlying mechanism. Manual Therapy. 2005;10:242-55.

O'Sullivan P. It's time for change with the management of non-specific chronic low back pain. Br J Sports Med. 2012;46:224-7

Pengel LH, Refshauge KM, Maher CG. Responsiveness of pain, disability, and physical impairment outcomes in patients with low back pain. Spine (Phila Pa 1976). 2004;29:879-83.

Roland M, Fairbank J. The Roland-Morris Disability Questionnaire and the Oswestry Disability Questionnaire. Spine (Phila Pa 1976). 2000;25:3115-24.

Roland M, Morris R. A study of the natural history of back pain. Part I: development of a reliable and sensitive measure of disability in low-back pain. Spine. 1983;8:141-4.

Ryoo JH. Model Selection with the Linear Mixed Model for Longitudinal Data. Multivar Behav Res. 2011;46:598624.

Sahrmann SA. Diagnosis and treatment of movement impairment syndromes. St.Louis: Mosby.; 2002.

Saner J, Kool J, de Bie RA, Sieben JM, Luomajoki H. Movement control exercise versus general exercise to reduce disability in patients with low back pain and movement control impairment. A randomised controlled trial. BMC Musculoskelet Disord. 2011;12:207.

Smith BE, Littlewood C, May S. An update of stabilisation exercises for low back pain: a systematic review with meta-analysis. BMC Musculoskelet Disord. 2014;15:416.

Son $\mathrm{H}$, Friedmann E, Thomas SA. Application of pattern mixture models to address missing data in longitudinal data analysis using SPSS. Nursing research. 2012;61:195-203.

Stanton TR, Henschke N, Maher CG, Refshauge KM, Latimer J, McAuley JH. After an episode of acute low back pain, recurrence is unpredictable and not as common as previously thought. Spine (Phila Pa 1976). 2008;33:2923-8.

Stratford P GC, Westaway M, Binkley J, . Assessing disability and change on individual patients: a report of a patient specific measure. Physiother Canada. 1995;47:258-63.

Tamcan O, Mannion AF, Eisenring C, Horisberger B, Elfering A, Muller U. The course of chronic and recurrent low back pain in the general population. Pain. 2010;150:451-7.

Turk DC, Melzack, R. Handbook of pain assessment: The Guildford Press; 2011.

Vibe Fersum K, O'Sullivan P, Skouen JS, Smith A, Kvale A. Efficacy of classification-based cognitive functional therapy in patients with non-specific chronic low back pain: a randomized controlled trial. Eur J Pain. 2013;17:916-28.

Von Korff M. Studying the natural history of back pain. Spine (Phila Pa 1976). 1994;19:2041S-6S.

Von Korff M, Dunn KM. Chronic pain reconsidered. Pain. 2008;138:267-76.

Waddell G. 1987 Volvo award in clinical sciences. A new clinical model for the treatment of low-back pain. Spine (Phila Pa 1976). 1987;12:632-44. 
Whaley MH. ACSM's Guidelines for exercise testing and prescription. In: Medicine ACoS, editor.: Lippincott Williams \&Wilkins; 2006.

Wiesinger GF, Nuhr M, Quittan M, Ebenbichler G, Wolfl G, Fialka-Moser V. Cross-cultural adaptation of the Roland-Morris questionnaire for German-speaking patients with low back pain. Spine (Phila Pa 1976). 1999;24:1099-103. 



\section{Chapter}

\section{The patient-specific functional scale - its construct validity and validity to change in patients with shoulder and low back pain}





\section{Introduction}

The use of assessment tools that can reflect the actual status or degree of restriction and which can measure change over time, is of crucial importance. For a therapist, it is essential to ascertain whether improvements in body function or structure also lead to increased activity and participation levels. Improvements in body functions and structures are predominantly assessed through physical testing; activity and participation are commonly measured using questionnaires. Scores gathered using these measurement tools allow comparison at a group level and enable therapists and researchers to "measure" the impact of a disease, its progression over time or the effect of an intervention on a patient group. Since questionnaires often contain very specific items related to certain activities, it is possible that some items might not be relevant to all patients in a group. In addition, the importance of the items could vary between patients or "prefixed" items may exclude activities of importance to certain patients. Therefore, patients may be required to score questions that are of higher of lower relevance to them. As a result, these standard questionnaires might not adequately reflect a patient's individual restrictions or the change in these restrictions over time.

In an attempt to solve this problem, the Patient-Specific Functional Scale (PSFS) was developed with the intention to monitor a patient's progress based on relevant restrictions chosen by the individual himself (1). The PSFS is comprised of 1 to 5 activities; each activity is rated on an 11-point numeric rating scale (NRS) from 0 (impossible to do) to 10 (fully capable). The PSFS is easy to administer, and takes only five to ten minutes to complete. However, the PSFS also has been used in the past by researchers to determine the current state of function and the development of activity restrictions over time at a group level. By choosing this approach, researchers have moved away from the originally intended individual focus of the instrument and applied the PSFS to situations for which it was not developed or validated.

From a test-theoretical perspective, there are numerous problems in deviating from the original construct. Firstly, the interpretation of an average score across self-selected activities by individuals is a challenge. How to define the underlying construct and next the outcome? For researchers and clinicians who are familiar with interpreting data on a clearly defined aspect of disability, it is tempting to interpret outcomes using the same approach; but one is, in fact, averaging apples and oranges. Another problem is that ceiling or floor effects could occur if a patient chooses activities at the baseline measurement that are out of the range of his individual capabilities over time, or when the initially chosen activities become increasingly irrelevant as a problem due to a patient's improved condition and reduction in complaints.

Despite these problems, which have not as yet been adequately addressed, several researchers have investigated the psychometric properties of the PSFS on a group level for a variety of musculoskeletal conditions. Results have been formulated as "promising", since the PSFS has been reported as having good construct validity, discriminant validity, 
and responsiveness (2-4). In our opinion, based on these results, testing psychometric properties and comparisons at a group level can be justified by defining the PSFS as an instrument assessing "activity restriction based on items selected by an individual patient" as the overriding construct. We hypothesized that specific musculoskeletal disorders (in our example subacromial shoulder pain and low back pain) lead to specific activity restrictions and specific pain patterns. Condition-specific outcome measures (CSOMs), in our case the Shoulder pain and Disability Index (SPADI) and the Roland \& Morris Disability Questionnaire (RMDQ), summarize these typical activities and include a range of tasks from easy to more difficult; the items included cover the whole range of items assumed to be relevant for a patient group, although not every item may be of equal importance to each individual patient. Therefore, we assume that most activity restrictions chosen by individual patients for their PSFS can be traced back to the items listed in the CSOMs. If this is the case, the PSFS could be approached as a construct and, because of the hypothesized close association between the operationalization of both types of measurement, assumed to deliver a relatively high correlation with the CSOM, especially in a cross-sectional analysis. Outcomes in the PSFS could indicate higher disability compared to CSOMs when patients choose activities at baseline that they will be unable to perform, but may depend on instructions given to the patient. With this background, the aims of this paper are threefold: in two groups of patients, suffering from either subacromial pain syndrome (SPS) or low back pain (LBP), and using the CSOM as an external standard comparator: 1. To test for possible floor and ceiling effects of the PSFS; 2 . To evaluate its construct validity compared to the CSOM; and 3. To assess the ability of the PSFS to detect changes over time.

\section{Methods}

Data was used from two different datasets; these were the results from randomized controlled trials investigating effects of physiotherapy interventions on SPS and LBP in primary care. A detailed description of the inclusion process, applied treatments and primary analyses can be found in the published study protocols $(5,6)$ and trial results $(7-10)$. Ethical approval was granted by the ethics committee of the Ludwig-Maximilians-University Munich, Germany (project-no. 018-10) (SPS trial), and the Swiss Ethics Committee KEK-ZH-NR: 2010-0034/5, (LBP trial). All patients in both trials gave informed consent. Datasets of the two samples were analyzed independently of each other.

\section{Dataset 1 -SPS patients}

Participants were recruited through referral for physiotherapy due to shoulder complaints. After baseline assessment they were randomly assigned to either an intervention 
or control group. The intervention group received exercise therapy plus manual therapy, while the control group received only exercise therapy. Baseline characteristics of the 90 participants included in the trial are presented in Table 1.

The primary outcome measure was the SPADI, a shoulder-specific, self-reported questionnaire measuring pain and disability (15). SPADI sub-scales for pain (items 1 to 5 ) and function (items 6 to 13) are scored from 0 to 100, with higher scores reflecting higher pain or disability levels. Total SPADI score was calculated by averaging scores of the two sub-scales. The SPADI has been shown to be valid and highly sensitive $(11,12)$. The German version of the SPADI has also been shown to have excellent reliability and internal consistency (13). The PSFS $(1,14)$ was also applied. Patients were instructed to choose 3 activities important to them, in which they were also impaired, and to rate their ability to perform them on an 11-point NRS from 0 (impossible to do) to 10 (fully capable). The average score across all activities was calculated. , For the sake of legibility, the PSFS has been rescaled in this paper, so that 0 now means "fully capable" and 10 means "impossible to do", in accordance with the other outcome measures used in this analysis. All measurement instruments were applied at baseline (BL), after 5 weeks (T1), 12 weeks (T2), and at one year follow-up (T3) (except for pain on the NRS).

\section{Dataset 2 - LBP patients}

A total of 106 patients with LBP, defined as pain persisting for longer than six weeks and with no radiating symptoms below the knee were included in the original LBP trial. Eligible patients presented with defined complaints associated with movement control impairment ( $\mathrm{MCl}$ ). Other inclusion criteria were, a score of at least two positive out of six movement tests (representing $\mathrm{MCl}$ ) and a minimal level of disability of 5 points on the RMDQ $(6,15)$. Participants were randomly allocated to either the intervention group that received an individual complaint-specific exercise program, or to a control group that received general exercise therapy. Baseline characteristics are presented in Table 1. 
Table 1. Baseline demographic data and baseline results for SPS and LBP patients initially included in the original trials (Mean (SD) if not otherwise stated)

\begin{tabular}{lll}
\hline & SPS $(n=90)$ & $\operatorname{LBP}(n=106)$ \\
\hline Age in years & $51.8(11.2)$ & $41.6(14.1)$ \\
Gender (female) \% $(n=)$ & $41.1(46)$ & $66.0(40)$ \\
Duration of the current episode in weeks & $33.9(42.8)$ & -- \\
Overall duration of complaints in years & $8.7(12.7)$ & $10.0(11.0)$ \\
SPADI/RMDQ total score & $40.4(17.0)$ & $8.7(3.3)$ \\
PSFS average score & $6.0(1.7)$ & $5.7(1.6)$ \\
GCPS total score & -- & $27.8(10.4)$ \\
GCPS sub-score disability & -- & $12.4(7.6)$ \\
FABQ total score & $32.7(17.4)$ & $32.2(14.7)$ \\
\hline
\end{tabular}

$\mathrm{SD}=$ standard deviation; SPADI = Shoulder pain and disability index; RMDQ = Roland Morris Disability Questionnaire; PSFS = Patient-specific functional scale; $F A B Q=$ Fear avoidance beliefs questionnaire; GCPS= graded chronic pain scale (total score: 70; pain intensity: 0-30; disability: 0-40)

Primary outcome measure was the PSFS $(1,14,16)$. Patients were given the same instructions as in the shoulder trial. A secondary outcome was the RMDQ, which measures LBPrelated disability. It consists of 24 dichotomous questions to be answered with either "yes" or "no", with a "yes" score meaning high disability. Reliability was shown to be high and construct and internal validity to be good, also for the German version (17-20). All outcomes were measured at baseline, at 9-12 weeks (T1), 6 months (T2), and at one year follow-up (T3).

An overview and description of all outcome measures for both datasets are provided in Table 2. For this study, we decided only to include those patients with complete data who are relevant to our analysis.

Table 2. Outcome measures used in the two trials

\begin{tabular}{|c|c|c|c|}
\hline Outcomes measures & Dimension & Scale & Scorings \\
\hline \multicolumn{4}{|l|}{ a) SPS } \\
\hline $\begin{array}{l}\text { Shoulder pain and } \\
\text { disability index (SPADI) }\end{array}$ & $\begin{array}{l}\text { SPS - related pain } \\
\& \text { activity } \\
\text { limitations }\end{array}$ & $\begin{array}{l}0-100 \text {, continuous } \\
0-100 \text {, continuous }\end{array}$ & $\begin{array}{l}\text { Items } 1-5 \text { scored on a } 100 \mathrm{~mm} \text { VAS } \\
\text { Items } 6-13 \text { scored on a } 100 \mathrm{~mm} \text { VAS } \\
\text { Mean of item scores. Higher scores mean } \\
\text { higher pain/disability. }\end{array}$ \\
\hline $\begin{array}{l}\text { Patient-specific } \\
\text { functional scale (PSFS) }\end{array}$ & $\begin{array}{l}\text { SPS - related } \\
\text { disability }\end{array}$ & $0-10$, continuous & $\begin{array}{l}11 \text { point visual numeric rating scale (end } \\
\text { descriptors of } 0=\text { impossible to do, } 10=\text { no } \\
\text { difficulties at all) }\end{array}$ \\
\hline \multicolumn{4}{|l|}{ b) $\angle B P$} \\
\hline $\begin{array}{l}\text { Patient-specific } \\
\text { functional scale (PSFS) }\end{array}$ & $\begin{array}{l}\text { LBP - related } \\
\text { activity limitations }\end{array}$ & $0-10$, continuous & $\begin{array}{l}11 \text { point visual numeric rating scale (end } \\
\text { descriptors of } 0=\text { impossible to do, } 10=\text { no } \\
\text { difficulties at all) }\end{array}$ \\
\hline $\begin{array}{l}\text { Roland-Morris disability } \\
\text { questionnaire (RMDQ) }\end{array}$ & $\begin{array}{l}\text { LBP - related } \\
\text { disability }\end{array}$ & $0-24$, continuous & $\begin{array}{l}\text { Dichotomous questions (yes=with disability, } \\
\text { no=no disability); Scores } 0-24 \text { (minimal } \\
\text { enrolment to trial RMDQ }=5 \text { ) }\end{array}$ \\
\hline
\end{tabular}

SPS = subacromial pain syndrome; LBP = low back pain; VAS = visual analogue scale; SPADI = shoulder pain and disability index; RMDQ = Roland-Morris disability questionnaire. 


\section{Data analysis and hypotheses}

Floor and ceiling effects

Since patients may be greatly restricted in their activities at the start of treatment, they may also have high scorings for their chosen activities on the PSFS. Data were checked for possible floor and ceiling effects by using stem-and-leaf-plots at every measurement point. Floor and/or ceiling effects were assumed when more than $15 \%$ of values were within $10 \%$ of the highest and/or lowest possible scores.

Hypothesis 1:

There are no floor or ceiling effects at any measurement point.

\section{Construct validity}

To test the construct validity of the PSFS we calculated correlations between the PSFS and SPADI or RMDQ, respectively, at every measurement point using Pearson's $r$. A high correlation was defined as $r \geq 0.75$, a moderate correlation as $r \geq 0.5$ and a low correlation as $r \geq 0.25$. High correlations were expected for baseline scorings because both measurements should reflect the current status of disability. For the consecutive time points T1, $\mathrm{T} 2$, and T3 progressively decreasing correlations were expected: from high at baseline to low at T3, especially in patients showing good improvement. This also is based on the fact that patients were not allowed to change the initially chosen PSFS activities over the 1year follow up period. Consequently, we expected that these activities would become increasingly irrelevant for patients as their health status improved over time.

Hypothesis 2a:

There is a high correlation between the PSFS and CSOMs (RMDQ/SPADI) at baseline.

Hypothesis 2b:

The correlation between the PSFS and the CSOMs (RMDQ/SPADI) measured at every follow-up point in a cross-sectional independent way (T1, T2, and T3) is lower than the correlation of the preceding point: r-values will decrease from high at baseline to moderate, and to low at T3.

\section{Validity to change}

To test the ability of the PSFS to detect change over time we calculated correlations between the change scores in the SPADI/RMDQ and the change scores in the PSFS for the following intervals: BL to $\mathrm{T} 1, \mathrm{~T} 1$ to $\mathrm{T} 2$, and $\mathrm{T} 2$ to $\mathrm{T} 3$. We expected that the correlation between change scores would be acceptable in the short term, but diverge over the longer term. Therefore, our third hypothesis was:

Hypothesis 3:

PSFS change scores show high correlations with both the SPADI and the RMDQ 
between $\mathrm{BL}$ to T1, moderate correlations between T1-T2, and low correlations between T2-T3.The CSOMs are used as external anchors.

\section{Results}

\section{Participants}

Complete datasets were available for 87 SPS-participants (96.7\%), and for 60 LBP-participants (56.6\%). Characteristics of both samples are described in Table 3.

Table 3. Baseline demographic data and baseline results for SPS and LBP samples included in this analysis (Mean (SD) if not otherwise stated)

\begin{tabular}{lll}
\hline & SPS $(n=87)$ & LBP $(n=60)$ \\
\hline Age in years & $52.0(11.4)$ & $41.8(13.9)$ \\
Gender (female) \% $(n=)$ & $49.4(43)$ & $38.3(23)$ \\
Duration of the current SPS episode in weeks & $33.6(43.5)$ & -- \\
Overall duration of complaints in years & $8.6(12.9)$ & $9.1(10.4)$ \\
SPADI/RMDQ total score & $41.0(17.0)$ & $8.7(3.3)$ \\
PSFS average score & $6.0(1.6)$ & $5.7(1.6)$ \\
GCPS total score & -- & $26.5(10.1)$ \\
GCPS sub-score disability & -- & $11.1(7.4)$ \\
FABQ total score & $32.0(17.2)$ & $29.3(13.8)$ \\
\hline
\end{tabular}

$\mathrm{SD}=$ standard deviation; SPADI = Shoulder pain and disability index; RMDQ = Roland Morris Disability Questionnaire; PSFS = Patient-specific functional scale; FABQ = Fear avoidance beliefs questionnaire; GCPS= graded chronic pain scale (total score: 70; pain intensity: 0-30; disability: 0-40)

\section{Floor and ceiling effects (hypothesis 1)}

No evidence of ceiling effects at any measurement point was found.

Floor effects were found in the SPS sample at T2 and T3, with $20.7 \%(n=18)$ and $48.3 \%$ $(n=42)$, respectively, within $10 \%$ of the lowest possible score. However, at T2 these participants had a mean (SD) SPADI score of 3.2 (4.0) points with only two participants scoring 10 points or higher. At T3, the mean SPADI score was 2.2 (3.3) with again only two participants scored 10 points or higher.

In the LBP sample floor effects were found at T1, T2, and T3, increasing from $21.6 \%$ $(n=13), 28.3 \%(n=17)$ to $43.3 \%(n=26)$, respectively; as seen in the SPS sample, the average scores of these patients on the RMDQ were also comparably low. Results are summarized in Table 4. 
Table 4. $n=(\%)$ of patients scoring within the highest and lowest $10 \%$ of the PSFS

\begin{tabular}{lllll}
\hline SPS sample $(n=87)$ & $\mathrm{BL}$ & $\mathrm{T} 1$ & $\mathrm{~T}$ & $\mathrm{T3}$ \\
\hline PSFS $\leq 1$ & $0(0)$ & $9(10.3)$ & $18(20.7)$ & $42(48.3)$ \\
SPADI mean (SD) score* & -- & -- & $3.2(4.0)$ & $2.2(3.3)$ \\
PSFS $>9$ & $5(5.8)$ & $0(0)$ & $2(2.3)$ & $2(2.3)$ \\
LBP sample $(n=60)$ & $\mathrm{BL}$ & $\mathrm{T} 1$ & $\mathrm{~T} 2$ & $\mathrm{~T} 3$ \\
PSFS $\leq 1$ & $0(0)$ & $13(21.6)$ & $17(28.3)$ & $26(43.3)$ \\
RMDQ mean (SD) score* & -- & $1.3(1.6)$ & $1.8(3.0)$ & $0.45(0.5)$ \\
& & & & $0(0)$ \\
\hline
\end{tabular}

$*=$ If more than $15 \%$ scored in the lower or higher $10 \%$ the mean (SD) SPADI/RMDQ score of this sub-sample is displayed.

\section{Construct validity (hypotheses $2 a$ and $2 b$ )}

The PSFS correlated well with SPADI and RMDQ at baseline. However, correlation coefficients were below our predefined cut-off level of $r \geq 0$.75. Our hypothesis $2 a$, therefore, had to be rejected.

Correlations for the time-points T1, T2 and T3 showed a progressive increase: we found the strongest correlations at T3, with $r=0.90$ in the SPS and $r=0.74$ in the LBP sample. This development was completely contrary to our stated hypothesis $2 \mathrm{~b}$. Based on these results, hypothesis $2 \mathrm{~b}$ also had to be rejected. Detailed results are displayed in Table 5.

Table 5. Correlations (Pearson`s $r$ ) between PSFS and condition-specific disability scores

\begin{tabular}{|c|c|c|c|c|c|c|c|c|c|c|}
\hline & $B L$ & $\mathrm{~T} 1$ & & $\mathrm{~T} 2$ & & T3 & $\begin{array}{l}\text { Change } \\
\text { BL-T1 }\end{array}$ & $\begin{array}{l}\text { Change } \\
\text { T1-T2 }\end{array}$ & $\begin{array}{l}\text { Change } \\
\text { T2-T3 }\end{array}$ & $\begin{array}{l}\text { Change } \\
\text { BL-T3 }\end{array}$ \\
\hline $\begin{array}{l}\text { Outcome } \\
\text { measure }\end{array}$ & $\begin{array}{l}\text { SPS \& } \\
\text { LBP }\end{array}$ & $\begin{array}{l}5 \\
\text { weeks }^{a} \\
\text { SPS }\end{array}$ & $\begin{array}{l}\text { 9-12 } \\
\text { weeks }^{a} \\
\text { LBP }\end{array}$ & $\begin{array}{l}12 \\
\text { weeks } \\
\text { SPS }\end{array}$ & $\begin{array}{l}6 \\
\text { months } \\
\text { LBP }\end{array}$ & $\begin{array}{l}12 \\
\text { months } \\
\text { SPS \& } \\
\text { LBP }\end{array}$ & & & & \\
\hline SPADI & 0.446 & 0.644 & & 0.805 & & 0.904 & 0.584 & 0.425 & 0.688 & 0.680 \\
\hline RMDQ & 0.301 & & 0.523 & & 0.651 & 0.743 & 0.497 & 0.542 & 0.176 & 0.552 \\
\hline
\end{tabular}

$\mathrm{BL}=$ baseline; $r$ = Pearson's correlation coefficient; ${ }^{\mathrm{a}}=$ post intervention; ${ }^{\mathrm{b}}=$ final follow $\mathrm{up} ; \mathrm{SPADI}=$ shoulder pain and disability index; RMDQ = Roland Morris disability questionnaire; PSFS = patient specific functional scale

Validity to change (hypothesis 3)

Here we expected decreasing correlation coefficients for change scores over time.

However, results did not support this hypothesis. Instead of decreasing correlation coefficients, we found alternating patterns which varied between samples. For the SPS $r$ values varied in an up-down sequence, with the strongest correlation for the change score between T2 and T3. The sequence for the LBP sample was more variable with a down-up-down pattern. Here, a very low correlation was found between T2 and T3. Results are displayed in Table 5. 


\section{Discussion}

The aim of this paper was to test the following hypotheses regarding the PSFS when compared to well-established CSOMs: no floor and ceiling effects, acceptable construct validity and validity to change in the short term. Other than for floor and ceiling effects our results showed opposite effects than we hypothesized, resulting in the rejection of our hypotheses regarding construct validity and validity to change.

These results demonstrate that the umbrella construct defined in the introduction must be doubted. The PSFS certainly does not reflect change on a group level in the same way that CSOMs do. The development over time of the correlations between PSFS and CSOMs has led us to conclude that the underlying constructs are different and, therefore, should not be used for the same purpose. Although the PSFS has been used in several studies as a secondary outcome measure to analyze longitudinal development of activity restrictions on a group level $(7,10,21-23)$ and seemed to perform well for this purpose, our data suggest that the underlying construct remains unclear. Therefore we cannot recommend the use of the PSFS on a group level (24).

Validity aspects of the PSFS also have been investigated by other authors. Hall et al. (25) investigated responsiveness of RMDQ and PSFS in patients with LBP and attested both outcome measures an "acceptable" responsiveness. In a first step, they calculated correlations between RMDQ/PSFS and the global perceived effect scale (GPE), which they used as an external reference standard for change. In a second step, they used a receiver operating characteristics (ROC) curve to assess responsiveness. This methodological approach basically provides information about the relationship between RMDQ/PSFS and GPE as an external indicator of change. Although GPE is often used for this purpose, GPE (change) scores might be more a reflection of the current health status than of true change and GPE might, therefore, be insufficient to serve as a valid external reference for change (26). Furthermore, when using ROC it is necessary to dichotomize the external change scores which leads to a loss of information on the magnitude of change (27). Our purpose was to analyze whether the PSFS was responsive relative to our CSOMs. The different approach to Hall et al. (25) may explain the difference in conclusions drawn from these results.

Abbott and Schmitt (2) investigated concurrent validity (which would be defined as construct validity in the absence of a gold standard according to Mokkink et al. (28)) and validity to change of the PSFS in a sample with mixed acute and chronic musculoskeletal disorders. According to our classification system for the correlation coefficient, they found a moderate correlation between PSFS and CSOMs at baseline in the subgroup with upper extremity disorders and a low correlation in the subgroup with LBP. Interestingly these correlations were stronger at 6-month follow-up, which were similar to the developments in our samples. The moderate correlations found for change scores between baseline and follow-up at 6 months were also similar to ours (between baseline and our 
last measurement point at 1 year), although the use of different time frames may complicate this comparison.

Similar results regarding construct validity were also found by Heldmann et al. (29) in LBP-patients. They used the Oswestry Disability Index as reference measure and found low correlations at baseline and moderate correlations at follow-up.

The fact that we obtained similar results to those of Abbott et al. (2) and Heldmann et al. (29) for patients with LBP and SPS has led us to the assumption that results could be independent of the population of interest. This assumption is underpinned by similar results found for lower extremity and neck disorders in the study of Abbott et al. (30), and by results from Stratford et al. who also found low correlations between change scores of PSFS and CSOM used in patients who underwent total knee arthroplasty (31). In our opinion, it is more likely that the similarity of results is mainly based on a basic difference between the constructs of the PSFS and CSOMs.

In another study Mannberg-Bäckman et al. (32) investigated the validity and sensitivity to change of the PSFS in a group of patients after proximal humerus fracture. Although $62 \%$ of the chosen items in the PSFS were also represented in the CSOM, correlation between the two instruments was low, whereas sensitivity to change was high in both instruments.

Resnik et al. (33) investigated responsiveness of several CSOMs and the PSFS in patients undergoing rehabilitation for upper limb prosthesis. The PSFS showed the largest effect sizes from all outcomes analyzed. This difference between PSFS and other outcomes was most obvious for long term results, where PSFS still showed significant changes while most of the other instruments did not. The authors concluded that the PSFS was one of the outcome measures most responsive to change. Heldmann et al. (29) also concluded that the PSFS is more responsive than the Oswestry Disability Index (ODI) because they found higher effect sizes for the PSFS. These results may also suggest that the PSFS measures a different construct than CSOMs. Besides, effect sizes are a reflection of quantitative properties and not necessarily of the validity of an instrument. These results suggest that larger effects can be reached when using the PSFS instead of a CSOM, but do not clarify the construct underlying the PSFS.

Originally the PSFS was developed to address individual activity restrictions, to prioritize treatment goals, and, last but not least, to keep patients motivated by working on personally important goals. We would agree with using the PSFS for these purposes. We also would agree with using the PSFS for risk assessment (34) and to monitor short-term effects in individual patients. The PSFS also may help to redirect focus towards function and ability rather than pain and disability in pain-focused patients.

Based on our data, we believe that the PSFS does not provide a clinically meaningful unidimensional scale comparable to a CSOM, because it aggregates not only heterogeneous functional items in one scale but also averages item-dependent scores. This makes it difficult to compare scores between patients. 


\section{Limitations}

Both of our samples contained mainly patients with chronic complaints and our results could be different from studies that investigate samples including acute patients.

A disadvantage of calculating correlations for assessing validity to change might be that different correlation coefficients may result from two samples drawn from the same population due to a different patient mix, even if the basic correlation between the two variables is the same. Furthermore, correlations measure the closeness to a linear relationship but the relationship between two measures may be close but non-linear.

Our values set for the Pearson's correlation coefficient for the acceptance of the stated hypotheses were to a certain degree subjective and certainly high and led to a more conservative interpretation of the results.

However, an important strength of our study is that two samples were analyzed from different populations and that we found similar results for both. In addition, we incorporated 4 measurement points in our analysis and were able to present the development of the relationship between the PSFS and the CSOMs over the time-frame of one year.

\section{Conclusion}

Possibly one of the biggest advantages of the PSFS in the clinical context is its dynamic adaptability to measure a patient's progress; using the PSFS as a longitudinal outcome would mean retaining the initially chosen activities and thus sacrificing this dynamic adaptability for the sake of standardization. If the goal is to assess the development of pathology or syndromes and to compare between patients on a group level, we would recommend relying on comprehensive CSOMs. 


\section{References}

1. Stratford PW, Gill C, Westaway MD, Binkley JM. Assessing disability and change on individual patients: a report of a patient specific measure. Physiother Can. 1995;47(4):258-63.

2. Abbott JH, Schmitt JS. The Patient-Specific Functional Scale was valid for group-level change comparisons and between-group discrimination. J Clin Epidemiol. 2014;67:681-8.

3. Hefford C, Abbott JH, Arnold R, Baxter GD. The Patient-Specific Functional Scale: Validity, Reliability, and Responsiveness in Patients With Upper Extremity Musculoskeletal Problems. J Orthop Sports Phys Ther. 2012;42:56-65.

4. Rosengren J, Brodin N. Validity and reliability of the Swedish version of the Patient Specific Functional Scale in patients treated surgically for carpometacarpal joint osteoarthritis. J Hand Ther. 2013;26:53-61.

5. Kromer TO, de Bie RA, Bastiaenen CHG. Effectiveness of individualized physiotherapy on pain and functioning compared to a standard exercise protocol in patients presenting with clinical signs of subacromial impingement syndrome. A randomized controlled trial. BMC Musculoskeletal Disorders. 2010;11:114.

6. Saner J, Kool J, Sieben JM, Luomajoki H. Movement control exercises versus general exercise to reduce disability in patients with low back pain and movement control impairment. A randomized controlled trial. BMC Musculoskeletal Disorders. 2011;12:207.

7. Kromer TO, de Bie RA, Bastiaenen CHG. Physiotherapy in patients with clinical signs of shoulder impingement syndrome: a randomized controlled trial. Journal of Rehabilitation Medicine. 2013;45:488- 97.

8. Kromer TO, de Bie RA, Bastiaenen CHG. Effectiveness and costs of physiotherapy in patients with shoulder impingement syndrome: 1-year follow up of a randomized controlled trial. Journal of Rehabilitation Medicine. 2014;46:1029-36.

9. Saner J, Sieben JM, Kool J, Luomajoki H, Bastiaenen CHG, De Bie RA. A tailored exercise program versus general exercise for a subgroup of patients with low back poain and movement control impairment: $A$ randomized controlled trial with one-year follow up. Manual Therapy. 2015;20:672-9.

10. Saner J, Sieben JM, Kool J, Luomajoki H, Bastiaenen CHG, De Bie RA. A tailored exercise program versus general exercise for a subgroup of patients with low back poain and movement control impairment: Shortterm results of a randomized controlled trial. Journal of Bodyworks \& Movement Therapies. 2016;20:189202.

11. Beaton DE, Richards RR. Measuring function of the shoulder. A cross-sectional comparison of five questionnaires. J Bone Joint Surg Am. 1996;78A:882-90.

12. MacDermid J, Solomon P, Prkachin K. The shoulder pain and disability index demonstrates factor, construct and longitudinal validity. BMC Musculoskeletal Disorders. 2006;7:12.

13. Angst F, Goldhahn J, Pap G, Mannion AF, Roach KE, Siebertz D, et al. Cross-cultural adaptation, reliability and validity of the German shoulder pain and disability index (SPADI). Rheumatology (Oxford). 2007;46:8792.

14. Horn KK, Jennings S, Richardson G, Vliet DV, FHeffod C, Abbott JH. The patient-specific functional scale: psychometrics, clinimetrics, and application as a clinical outcome measure. J Orthop Sports Phys Ther. 2012;42:30-42.

15. Luomajoki H, Kool J, de Bruijn ED, Airaksinen O. Movement control tests of the low back; evaluation of the difference between patients with low back pain and healthy controls. BMC Musculoskeletal Disorders. 2008;9:170.

16. Hall AM, Maher CG, Ferreira ML, Costa LO. The patient-specific functional scale is more responsive than the Roland Morris disability questionnaire when activity limitation is low. Eur Spine J. 2011;20:79-86.

17. Roland M, Morris R. A study of the natural history of back pain. Part I: development of a reliable and sensitive measure of disability in low-back pain. Spine. 1983;8:141-4.

18. Wiesinger GF, Nuhr M, Quittan M, Ebenbichler G, Wolfl G, Fialka-Moser V. Cross-cultural adaptation of the Roland-Morris questionnaire for German-speaking patients with low back pain. Spine. 1999;11:1099-103.

19. Roland M, Fairbank J. The Roland-Morris Disability Questionnaire and the Oswestry Disability Questionnaire. Spine. 2000;25:3115-24. 
20. Klasen BW, Hallner D, Schaub C, Willburger R, Hasenbring M. Validation and reliability of the German version of the Chronic Pain Grade questionnaire in primary care back pain patients. Psychosocial Medicine. 2004;1:Doc07.

21. Costa LO, Maher CG, Latimer J, Hodges PW, Herbert RD, Refshauge KM, et al. Motor control exercises for chronic low back pain: a randomized placebo-controlled trial. Physical Therapy Journal. 2009;89(12):127586.

22. Brennan KL, Allen BC, Maldonado YM. Dry Needling Versus Cortisone Injection in the Treatment of Greater Trochanteric Pain Syndrome: A Noninferiority Randomized Clinical Trial. J Orthop Sports Phys Ther. 2007;47(4):232-9.

23. Ferreira G, Stieven F, Araujo F, Wiebusch M, Rosa C, Plentz R, et al. Neurodynamic treatment did not improve pain and disability at two weeks in patients with chronic nerve-related leg pain: a randomised trial. Journal of Physiotherapy. 2016;62:197-202.

24. Kyte DG, Calvert M, van der Wees PJ, ten Hove D, Tolan S, Hill JC. An introduction to patient-reported outcome measures (PROMs) in physiotherapy. Physiotherapy. 2015;101:119-25.

25. Hall MA, Maher CG, Latimer J, Ferreira ML, Costa LOP. The patient-specific functional scale is more responsive than the Roland Morris disability questionnaire when activity limitation is low. Eur Spine J. 2011;20:79-86.

26. Kamper SJ, Ostelo RWJG, Knol DL, Maher CG, De Vet HCW, Hancock MJ. Global perceived effect scale provides reliable assessments of health transition in people with musculuskeletal disorders, but ratings are strongly influenced by current status. J Clin Epidemiol. 2010;63:760-6.

27. Husted JA, Cook RJ, Farewell VT, Gladman DD. Methods for assessing responsiveness: a critical review and recommendations. J Clin Epidemiol. 2000;53(459-468).

28. Mokkink LB, Terwee CB, Patrick DL, Alonso J, Stratford PW, Knol DL, et al. The COSMIN study reached international consensus on taxonomy, terminology, and definitions of measurement properties for healthrelated patient-reported outcomes. J Clin Epidemiol. 2010;63:737-45.

29. Heldmann P, Schöttker-Köninger T, Schäfer A. Cross-cultural Adaption and Validity of the "Patient Specific Functional Scale. International Journal of Health Professionals. 2015;2(1):73-82.

30. Abbott JH, Schmitt JS. Minimum important difference for the patient-specific functional sclae, 4 region-specific outcome measures, and the numeric pain rating sclae. J Orthop Sports Phys Ther. 2014;44:560-4.

31. Stratford P, Kennedy DM, Wainwright AV. Assessing the Patient-Specific Functional Scale's Ability to Detect Early Recovery Following Total Knee Arthroplasty. Phys Ther. 2014;94(6):838-44.

32. Mannberg-Bäckman S, Stråt S, Ahlström S, Brodin N. Validity and sensitivity to change of the Patient Specific Functional Scale used during rehabilitation following proximal humeral fracture. Disabil Rehabil. 2016;35(8):487-92.

33. Resnik L, Borgia M. Responsiveness of outcome measures for upper limb prosthetic rehabilitation. Prosthetics and Orhtotics International. 2016;40(1):96-108.

34. Hinami K, Alkhalil A, Chouksey S, Chua J, Trick WE. Clinical significance of physical symptom severity in standardized assessments of patient reported outcomes. Qual Life Res. 2016;25:2239-43. 


\section{Chapter}

Patients' strategies and barriers to successful long-term adherence to home-based exercise programmes in physiotherapy 


\section{Abstract}

Background: Adherence to an exercise programme impacts the outcome of physiotherapy treatment in patients with non-specific low back pain.

Objectives: The aim of this study was to explore the strategies and barriers to long-term adherence to such exercise programmes from the patients' perspective.

Design: This qualitative study was embedded in a prior randomised controlled trial (RCT) which compared the effectiveness of two types of exercise programme on patients with nonspecific low back pain.

Method: Open-ended questionnaires of 44 participants were analysed using thematic analysis.

Results/findings: Patients' perceptions include the following themes: 1 ) the role of good knowledge in long-term exercise adherence; 2) from knowledge to practise - strategies and barriers; 3 ) the role of the perceived effects of exercise.

Conclusions: Results support the hypothesis that adherence can be improved through good knowledge of the exercises and their correct performance, combined with self-initiated training strategies. Prior, individually supervised physiotherapy treatment that includes the coaching of strategies for post-treatment exercise is recommended.

Keywords: exercise therapy, exercise adherence, movement control, low back pain, patients' strategies, qualitative research 


\section{Introduction}

We recently reported on a randomised controlled trial (RCT) (see Box 1) which tested the effectiveness of a specifically tailored exercise programme on a subgroup of patients diagnosed as suffering from NSLBP with $\mathrm{MCl}$ (Refxxx,yyy,zzz). $\mathrm{MCl}$ is defined as mechanically induced pain accompanied by movement abnormalities. The diagnosis was assessed by means of a series of functional tests, as described by Luomajoki $(1,2)$. The RCT revealed no difference between the tailored programme compared to a general exercise programme on all outcome measurements. However, both programmes resulted in significant improvements in function, disability and pain, which were retained over the course of one year following treatment. In this paper we report on an analysis of the embedded qualitative data, with the aim of exploring the strategies and barriers which influence the successful application of long-term, self-directed exercise programmes, as seen from the patients' perspective.

\section{Box 1: Short description of the RCT}

Patients $(n=106)$ who participated in the RCT were randomised into two exercise groups (movement control exercise $=M C / n=52$; general exercise $=G E / n=54$ ). Both groups were treated in individual treatment sessions of 30 minutes, ideally twice per week, over a period of nine to 12 weeks. Patients were strongly encouraged to exercise further at home over the following year. Thirteen physiotherapy clinics and practices in the German part of Switzerland participated in the RCT, while thirty-three therapists provided treatments. The concept of the MC treatment included a specifically tailored exercise programme directed at the diagnosed movement abnormalities, in order to eliminate these irregularities and normalise the movements. Initially, patients learned to control movements in closed chain positions, following which they progressed to open chain positions and increased loading. Posture and movement awareness were practised. Strength training was allowed once movement control had been achieved. In the GE group, the exercises were following a prescheduled standardised programme and aimed at improving general endurance and the strength of the lumbar and pelvic regions and legs.

Follow-up measurements were taken post-treatment, at six and 12 months. The primary outcome measurement was the Patient Specific Function Scale (PSFS); secondary measures were the Roland Morris Disability Questionnaire (RMDQ) and the Graded Chronic Pain Scale (GCPS) for disability and pain.

For home-based, unsupervised exercise programmes to be effective, patient adherence is of utmost importance, but is nevertheless hard to achieve. Previous systematic reviews of qualitative and quantitative studies have described barriers to exercise adherence (3), reviewed patients' perceptions towards exercise (4) and outlined interventions to improve adherence (5). They concluded that there is a lack of research investigating barriers 
to treatment adherence. In addition, they found large variations in the methods to improve adherence to exercise as well as in the measurement of exercise adherence $(6,7)$. Patients generally show preference for a supervised exercise programme over receiving exercise advice alone (4). It is unclear how people continue to exercise on their own once supervised training in therapy sessions has ended.

In a study among low back pain patients who participated in a stabilising exercise programme, increasing participants' knowledge and understanding was identified as a key theme in their treatment (8). However, the question remains as to which kind of knowledge is important and how patients successfully apply this knowledge in their daily lives on a long-term basis, and/or which factors prevent them from doing so.

This study explores the influences which either support or act as barriers to exercise adherence from the patient perspective. Specific focus is put on the role of increasing knowledge and understanding regarding the relevant concepts to apply a long-term, selfdirected exercise programme.

\section{Methods}

\section{Ethics}

The research was approved by the Swiss Ethics Committee (XXX) and registered with Current Controlled Trials (YYY). All patients in the study were informed of the aim and the protocol of the study and gave their written consent. For reporting, we followed the COREQ (COnsolidated criteria for REporting Qualitative research) (9).

\section{Data collection}

The questionnaire used was part of the final follow-up assessment of the RCT, which took place one year after enrolment. Forms were sent out to the patients and returned by postal mail. Due to the large amount of other measurements, a comprehensive set of three open-ended questions, based on factors identified in previous studies, was used. Sufficient space was provided for extensive answers $(3,8)$ :

Question 1: "Describe if and how the physiotherapy treatment helped you in the past year"

Question 2: "What did you learn in your physiotherapy treatment? Describe in your own words."

Question 3: "Describe if and how you implemented your new knowledge in your daily life. Describe in your own words."

The return process was supported through a reminder via telephone contact by a research assistant. 


\section{Data analysis}

Coding was carried-out by three of the authors, JS1 (physiotherapist, primary researcher of the RCT), EB (PhD, human movement scientist, independent researcher) and JS2 (PhD, human movement scientist, RCT supervisor).

Data was analysed using thematic analysis, which allows for the application deductively with pre-existing themes and codes, based on a theoretical hypothesis (10).

An initial categorising system was established by JS1 based on literature and was used for the preliminary template of themes. All three researchers were in agreement. A sample of 10 questionnaires were read and re-read separately by JS1, EB and JS2, who identified new themes and sub-themes. These (sub)themes were discussed and a code list was created. Another 3 sets of 10 questionnaires were coded and agreed on in alternating pairs of coders. Using this process the final coding list was developed.

The final coding list was used to code the questionnaires of patients from various clinical settings and both treatment groups, including recoding the questionnaires used to create the coding list. Coding led to little disagreement and consensus was always reached through discussion. After coding and discussing 44 questionnaires (consecutive patients 7-65) we reached saturation. The results of 14 participants were missing (withdrawn or non-response) during the data collection within the RCT.

Atlas.ti 7.0 was used for analysis.

\section{Results}

\section{Participants}

The sample of 44 patients ( $M C=19 ; \mathrm{GE}=25$ ) represents $41 \%$ of the 106 participants analysed during the RCT. See Table 2 for patient characteristics.

The median age of the sample was 43 years; mean pain duration of NSLBP was 9.5 years. Sports participation, as a measure of the general activity level before intervention, differed between MC and GE, with more MC participants reporting "no sport" ( $M C=44.5 \%$; $G E=26.9 \%$ ). Baseline assessment showed a mean disability of 6.1 (scale 010 , lower score means greater disability) as measured by the Patient-Specific Functional Scale (PSFS) and a score of 9.25 (scale 0-24, higher score means greater disability) on the Roland Morris Disability Questionnaire (RMDQ).

Characteristics of both qualitative samples are similar to the group data in the total RCT.

\section{Adherence and satisfaction with exercise}

Participants in the RCT were encouraged to do their recommended exercises at least twice per week over the course of the follow-up year. One year after enrolment, $46 \%$ of 
all participants reported that they had adhered to this exercise frequency or even exercised more often. Patients' satisfaction with their treatment after one year was rated high to very high ( 8 or more on an 11 point Likert scale) by $80 \%$ of the patients (11).

Table 1: Baseline characteristics of participants taking part in qualitative research design and full sample of the randomised controlled trial

\begin{tabular}{|c|c|c|c|c|c|c|}
\hline & $M C$ & & $\mathrm{GE}$ & & Both groups & \\
\hline & $\begin{array}{l}\text { Qualitative } \\
\text { sample }\end{array}$ & Total RCT & $\begin{array}{l}\text { Qualitative } \\
\text { sample }\end{array}$ & Total RCT & $\begin{array}{l}\text { Qualitative } \\
\text { sample }\end{array}$ & Total RCT \\
\hline$n$ & 18 & 52 & 26 & 54 & 44 & 106 \\
\hline Gender, female, $\mathrm{n}$ & 8 & 16 & 11 & 24 & 19 & 40 \\
\hline Age, $y$, mean (SD) & $43.1(12.5)$ & $42.8(13.8)$ & 44.9 (13.9) & $40.5(14.7)$ & $44.2(13.1)$ & $41.6(14.1)$ \\
\hline $\begin{array}{l}\text { Pain duration, } y \text {, } \\
\text { mean }\end{array}$ & 11 & 11.6 & 8 & 8.4 & 9.5 & 10.0 \\
\hline \multicolumn{7}{|c|}{ Sport participation n (\%) } \\
\hline no Sport & $\begin{array}{l}8 \\
(44.5 \%)\end{array}$ & $19(36.5 \%)$ & $\begin{array}{l}7 \\
(26.9 \%)\end{array}$ & $16(29.6 \%)$ & 15 (34.1\%) & $\begin{array}{l}35 \\
(33.1)\end{array}$ \\
\hline $1-2 \times$ week & $\begin{array}{l}6 \\
(33.5 \%)\end{array}$ & $21(40.4 \%)$ & $11(42.3 \%)$ & $23(42.6 \%)$ & $\begin{array}{l}17 \\
(38.6 \%)\end{array}$ & $44(41.5 \%)$ \\
\hline$>2 \times$ per week & $\begin{array}{l}4 \\
(22.2 \%)\end{array}$ & $10(19.2 \%)$ & $\begin{array}{l}6 \\
(23.1 \%)\end{array}$ & $13(24.1 \%)$ & $10(22.7 \%)$ & $23(21.7 \%)$ \\
\hline PSFS mean (SD) & $5.9(2.1)$ & $4.3(1.9)$ & $6.4(2.0)$ & $4.0(2.0)$ & $6.1(2.0)$ & $4.2(2.0)$ \\
\hline RMDQ mean (SD) & $9.7(3.2)$ & $9.1(3.2)$ & $8.9(4.1)$ & $8.2(3.3)$ & $9.25(3.8)$ & $8.6(3.3)$ \\
\hline
\end{tabular}

MC: Movement control group GE: General exercise group

PSFS: Patient-Specific Functional Scale self-score activity mentioned first, ranging from 0 (unable to perform) to 10 (able to perform without difficulties)

RMDQ: Roland Morris Disability Questionnaire score, ranging from 0 (no difficulties) to 24 (extreme difficulties) SD: standard deviation

The analyses of the qualitative data let to four major themes: the role of knowledge in long-term adherence to exercise; strategies to support exercise adherence; barriers to exercise adherence; and the role of the perceived effects of treatment. The results for each theme are presented below with illustrative quotes. Participants are identified by the ID codes of the RCT and group allocation (Patient 25 refers to the $25^{\text {th }}$ patient enrolled, GE refers to General Exercise group, MC refers to Movement Control group).

The role of knowledge in long-term adherence to exercise

With this topic, we explored how knowledge about exercise was perceived and whether new knowledge was accumulated during the year of training; as well as which knowledge remained of interest after one year and whether it had an impact on exercise adherence. 
Participants regarded the perceived relevance of exercise as their main knowledge gain. They learned by experience about the association between exercise and the prevention/resolution of their back problems.

"If I do my exercises regularly, I don't have low back pain. As soon as I become negligent, low back pain returns." (Patient 25, GE)

"As soon as I feel my back, I know which exercises I have to do in order to get rid of it." (Patient 7, MC)

Participants in both groups clearly described that they perceived that the exercises they learned were targeted at improving their back pain problem:

"I learned, to feel/to train the low back muscles goal-oriented and intensive." (Patient 46, GE)

"The exercises were always targeted at my problem and not following a randomly applied scheme." (Patient 31, MC)

"I do goal-oriented exercises once a week." (Patient 17, MC)

Confidence in knowing the correct performance of the exercises was important to all participants. Several participants mentioned that training under the guidance of the physiotherapist, the repetitions and corrections increased their knowledge of the exercises. Participants in the GE group reported that they had learned new exercises.

"I received precise instructions on how to perform the exercise" (Patient 34, MC)

"I trained the correct performance of the exercise." (Patient 25, GE)

"I got to know new exercises." (Patient 20, GE)

"We created a simple exercise plan together." (Patient 25, GE)

A good knowledge of the factors which aggravated their low back pain was frequently reported by all participants. Amongst other factors, sitting and lifting heavy loads were mentioned. The GE group participants referred more to strength training to overcome their deficits; the MC participants showed a trend towards regarding movement as better than static loading. These arguments are in line with the recommendations in the respective training programmes.

"I learned that strengthening the abdominal and back muscles is important." (Patient 61, GE)

"I learned that movement helps. Sitting is not good." (Patient 38, MC)

"I learned the correct behaviour and movement." (Patient 67, MC) 
"I broke with the habit of sitting incorrectly. I learned the correct way of lifting loads. I listen to my back more often and end activities earlier, if they burden my back." (Patient 13, GE)

Interestingly, knowledge of their diagnosis, possible causes of LBP or detailed knowledge of anatomy was not mentioned by any patient.

\section{Strategies to support exercise adherence}

Given that patients had learnt new knowledge or skills, we were interested to learn how and how often they were applying them; which strategies they had developed to include their exercise programmes into their daily lives.

Of general importance for the participants to be able to include their exercises into daily lives was their simplicity. They mentioned that the exercises were easy to perform at home and needed no additional equipment.

"I can repeat the exercises during the day. They are simple, but very efficient." (Patient 24, MC)

"The exercises shown can easily be done at home." (Patient 44, GE)

"I can do the exercises during my jogging in the forest." (Patient 48, GE)

A self-initiated participation in some form of structured, organised training helped participants to perform the exercises on a regular basis over the course of the year. Many participants reported this strategy and, it should be noted, that this advice was not part of the study protocol. Regularity in performing the exercises was often mentioned, together with discipline. The combination of general training and specific exercises made it easier to maintain discipline and acted as a reminder to do the exercises. They included their own exercises into other programmes of general fitness, back classes or general activity groups.

"If possible (absences) I visit a strength training twice per week." (Patient 51, GE)

"I perform back stability exercises in my fitness training 2-3 times a week. This is what I need." (Patient 27, MC)

The vast majority of participants mentioned the positive impact of the physiotherapy treatment on the correct performance of daily tasks. This comment was often mentioned in combination with an increased awareness of posture and the body in general, and also with achieved ergonomic changes. While participants in the GE group mentioned the correct performance of activities more often, the participants in the MC group reflected more often on improvement of their body posture. Several mentioned that they followed the general advice to sit, stand and lift correctly. To know how to retain good posture in aggravating situations was perceived as a benefit.

"I take care of how I walk and sit." (Patient 36, GE) 
"I'm standing the whole working day, I'm using a step now, where I can relax my legs." (Patient 54, MC)

"I'm more conscious of my posture, this means to correct it." (Patient 35, GE)

"I'm standing more consciously and also more relaxed." (Patient 43, MC)

Only in the GE group was the implementation of physical training into daily life perceived as increased general physical activity. They directly connected the physiotherapy intervention to their more active life style.

"I also do more sports and think this helps my back too." (Patient 47, GE)

"I climb stairs instead of using the lift or escalator." (Patient 20, GE)

\section{Barriers to exercise adherence}

Only a few participants actually reported inconsistent or absent exercise behaviour. This could be due to the fact that we did not explicitly ask for difficulties or barriers in the questionnaire. However, a few spontaneously mentioned lack of time or difficulty in including the exercises into their daily activities.

"I should take more time to do exercises." (Patient 42, MC)

"Rarely applicable, my possibilities to move are limited to work space, coffee area and WC." (Patient 38, MC)

"I can follow it partially, but I keep forgetting it from time to time." (Patient 21, $\mathrm{MC})$

One patient mentioned that he/she can't remember the exercises.

"I can't remember which exercise I should do." (Patient 56, GE)

Two patients mentioned that they no longer do their exercises without specifying a reason.

The role of perceived effects of treatment

This theme looks at which effects of treatment experienced were important to the patient and the connection between the perceived effects of the treatment and adherence to exercise afterwards.

Most often mentioned by our patients was physical "gain", described as: more muscle strength, elasticity or relaxation.

"I have the feeling, that my back is stronger and I notice it in my work." (Patient 47, GE)

"After treatment I very often had a good feeling in my back (relaxed low back and increased flexibility)." (Patient 10, GE) 
If our patients described physical relief they always mentioned pain. Apparently, and understandably, this was the major symptom of importance.

"When I started the therapy, it helped me a lot. At the end I was pain free." (Patient 23, MC)

"It did help me a lot, after the treatment my back pain only returned 1-2x per month." (Patient 29, GE)

The perceived effect of pain relief during treatment is a major motivator for exercise adherence.

"As soon as I notice my back, I know exactly which exercises I have to do to abolish my pain." (Patient 7, MC)

"It is good to quickly do the exercises, as soon as I feel the first signs of pain." (Patient 54, MC)

Some patients reported a very limited, short term effect of the treatment on their pain.

"The exercises helped for a very short time only." (Patient 14, MC)

"Good during the therapy sessions, but no remarkable long-term effect." (Patient 18, GE)

Two patients directly connected the limited effect to their non-adherence to exercise.

"Unfortunately not much effect, because I don't remember the exercises." (Patient 56, MC)

The perception of no effect on their pain was reported by three patients. They discontinued the exercises but kept in mind some advice from the therapy sessions.

"Did not help at all. If my back pain gets worse, I move more consciously as my physiotherapist recommended." (Patient 15, GE)

“Didn't help, pain still the same. I improved my posture." (Patient 49, MC) 


\section{Discussion}

More information on patients' perceptions of their experiences with exercise programmes for NSLBP may help to focus future physiotherapy treatments on factors which support long-term adherence to exercise. The results of this study point to gained confidence and a self-directed strategy as being the most successful.

\section{Confidence about the exercises and their performance is important}

If increased knowledge and perceived effects lead to self-empowerment and raised confidence in their own abilities, these steps would subsequently explain the high adherence rate in this study. The patient group gained confidence on several levels of perception: firstly, they highly valued the introduction to new exercises, the corrections and the control by their physiotherapist; secondly, their confidence increased due to the perceived effects of the exercises - the main perceived effects were physical gains, such as increased muscle power, flexibility, relaxation and general physical fitness; thirdly, patients noticed an association between the regular performance of exercises and less back pain; and lastly, confidence and security in the performance of their daily tasks was also increased.

Lack of confidence about the correct performance of exercises has been described previously as a main barrier to successful adherence $(3,4,12-14)$. This study supports these findings. Patients need confidence about how to correctly perform exercises and, therefore, require the support of trained instructors $(4,8,12,13)$. Easily-learned exercises were also easier to perform at home. As soon as the performance of exercises was connected to a perceived positive effect, our patients developed strategies to adhere to the programme. The same pattern applies to the knowledge of aggravating factors and the performance of the activities of daily life. Knowledge and individual successful experiences lead to behavioural change. A recent study with a protocol similar to the present study, rated the effect of performance in everyday life even higher than the applied exercises (15). This raises the question as to whether an additional behavioural approach would be beneficial. We agree with O'Keefe (2016), who compared the effects of physical interventions with behavioural/psychological informed interventions and combined interventions in a meta-analysis. They found no clinically important difference and commented that "the decision to choose a combined intervention should be balanced against the time and resources available" (16). Patients in the present study were able to successfully develop strategies to confidently change their performance in daily life after following simple instructions and experiencing the benefits. We recommend that exercise programmes should include easy, supervised training until the patients feel confident to continue by themselves. Additional behavioural treatment modalities may in that case not be necessary. 


\section{Individualised approach counts}

Patients perceived the exercises as individualised to their life situation and targeted to their back pain problem. These factors help to improve adherence to exercise $(3-5,12$, $14,17,18)$. The fact that patients in both groups reported perceived individualisation, when in reality the GE group followed a pre-set programme, gives rise to the speculation that patients and physiotherapists have different concepts of individualisation. Patients describe a certain pride in being able to perform their exercises correctly, regularly and with discipline, and also relating their new behaviour and their recognition of the aggravating factors. But it seems that which kind of exercises they should be doing are of no importance. It is the professional physiotherapist who makes this decision. It may be the way how the instructions to do the exercises are imparted which leaves an individualised impression, rather than the choice of exercises itself. The often overlooked therapist-patient relationship and communications skills can make a difference to successful adherence $(19,20)$.

\section{Self-organised training helps}

Strategies to overcome barriers to adherence, such as a perceived lack of self-discipline, lack of time or forgotten exercises, vary. Unexpectedly, many patients mentioned the inclusion of the exercises into a self-organised physical training format. Some trained in groups, some in individual fitness sessions and others in regular sports activities, such as jogging or walking. This need for further support has been described before $(4,12)$. Coaching patients at the end of physiotherapy training to find an appropriate and feasible long-term commitment to physical training is helping patients to adhere to their prescribed exercises. Furthermore, a good coaching process initiates self-efficacy and independence from the medical interventions which have been associated with positive treatment outcome (21).

\section{Strength and limitations}

The present study provides insight into long-term exercise programmes. However, patients received additional support through the procedures of the RCT. Phone calls, which were mainly aimed at optimising data collection, may have given additional encouragement to continue with the exercise instructions. Furthermore, we can only report the results of participants who finished the RCT and our results may be prone to some selection bias. Lastly, our results are based on self-reported written data which did not allow further elaboration. In terms of generalisability, this data applies to patients with NSLBP who were motivated enough to actively participate in an RCT focusing on exercise. It could be of interest for future research to explore whether the reported factors also apply to other conditions which require regular exercise and to populations who are not particularly interested in exercising. 
Embedding qualitative research into RCTs generates considerable evidence on the effectiveness of health interventions (22). The present study provides the means for interpretation of the results and describes contextual findings which can be of use in exercise treatments and future trials.

\section{Conclusion}

The results of this study indicate that gaining the confidence to exercise correctly has a high impact on long-term adherence to exercise. Easily-performed exercises brought noticeable benefits and supported; firstly, adherence to exercise; and, secondly, influenced performance in everyday life on a long-term basis. A successful adherence to long-term exercise appears to be best achieved by the inclusion of the exercises into a self-initiated organised training regimen. Future therapies should include coaching towards this strategy.

These insights enhance interpretation of the quantitative results and may be valuable in developing future long-term strategies which are of importance for patients with low back pain. 


\section{References}

1. Luomajoki H, Kool J, de Bruin ED, Airaksinen O. Movement control tests of the low back; evaluation of the difference between patients with low back pain and healthy controls. BMC Musculoskelet Disord. 2008;9:170. Epub 2008/12/26. doi: 1471-2474-9-170 [pii] 10.1186/1471-2474-9-170. PubMed PMID: 19108735; PubMed Central PMCID: PMC2635372.

2. Luomajoki H, Kool J, de Bruin ED, Airaksinen O. Improvement in low back movement control, decreased pain and disability, resulting from specific exercise intervention. Sports Med Arthrosc Rehabil Ther Technol. 2010;2:11. Epub 2010/04/27. doi: 1758-2555-2-11 [pii] 10.1186/1758-2555-2-11. PubMed PMID: 20416091; PubMed Central PMCID: PMC2873574.

3. Jack K, McLean SM, Moffett JK, Gardiner E. Barriers to treatment adherence in physiotherapy outpatient clinics: a systematic review. Man Ther. 2010;15(3):220-8. doi: 10.1016/j.math.2009.12.004. PubMed PMID: 20163979; PubMed Central PMCID: PMC2923776.

4. Slade SC, Patel S, Underwood M, Keating JL. What are patient beliefs and perceptions about exercise for nonspecific chronic low back pain? A systematic review of qualitative studies. Clin J Pain. 2014;30(11):9951005. doi: 10.1097/AJP.0000000000000044. PubMed PMID: 24300225.

5. Jordan JL, Holden MA, Mason EE, Foster NE. Interventions to improve adherence to exercise for chronic musculoskeletal pain in adults. Cochrane Database Syst Rev. 2010(1):CD005956. doi: 10.1002/14651858.CD005956.pub2. PubMed PMID: 20091582.

6. McLean S, Holden MA, Potia T, Gee M, Mallett R, Bhanbhro S, et al. Quality and acceptability of measures of exercise adherence in musculoskeletal settings: a systematic review. Rheumatology (Oxford). 2017;56(3):426-38. doi: 10.1093/rheumatology/kew422. PubMed PMID: 28013200.

7. Hall AM, Kamper SJ, Hernon M, Hughes K, Kelly G, Lonsdale C, et al. Measurement tools for adherence to non-pharmacologic self-management treatment for chronic musculoskeletal conditions: a systematic review. Arch Phys Med Rehabil. 2015;96(3):552-62. doi: 10.1016/j.apmr.2014.07.405. PubMed PMID: 25108098.

8. Sokunbi $O$, Cross V, Watt $P$, Moore A. Experiences of individuals with chronic low back pain during and after their participation in a spinal stabilisation exercise programme - a pilot qualitative study. Man Ther. 2010;15(2):179-84. doi: 10.1016/j.math.2009.10.006. PubMed PMID: 19939723.

9. Tong A, Sainsbury P, Craig J. Consolidated criteria for reporting qualitative research (COREQ): a 32-item checklist for interviews and focus groups. International journal for quality in health care : journal of the International Society for Quality in Health Care / ISQua. 2007;19(6):349-57. doi: 10.1093/intqhc/ mzm042. PubMed PMID: 17872937.

10. Braun VC, V. . Successful qualitative research: A practical guide for beginners.: London: Sage.; 2014.

11. Saner J, Kool J, Sieben JM, Luomajoki H, Bastiaenen CHG, de Bie RA. A tailored exercise program versus general exercise for a subgroup of patients with low back pain and movement control impairment: A randomised controlled trial with one-year follow-up. Manual Therapy. 2015;20(5):672-9. doi: 10.1016/j.math.2015.02.005. PubMed PMID: WOS:000361773900006.

12. Palazzo C, Klinger E, Dorner V, Kadri A, Thierry O, Boumenir Y, et al. Barriers to home-based exercise program adherence with chronic low back pain: Patient expectations regarding new technologies. Annals of physical and rehabilitation medicine. 2016;59(2):107-13. doi: 10.1016/j.rehab.2016.01.009. PubMed PMID: 27050664.

13. Escolar-Reina P, Medina-Mirapeix F, Gascon-Canovas JJ, Montilla-Herrador J, Jimeno-Serrano FJ, de Oliveira Sousa SL, et al. How do care-provider and home exercise program characteristics affect patient adherence in chronic neck and back pain: a qualitative study. BMC Health Serv Res. 2010;10:60. doi: 10.1186/1472-6963-10-60. PubMed PMID: 20219095; PubMed Central PMCID: PMC2847560.

14. Slade SC, Molloy E, Keating JL. 'Listen to me, tell me': a qualitative study of partnership in care for people with non-specific chronic low back pain. Clinical rehabilitation. 2009;23(3):270-80. doi: 10.1177/0269215508100468. PubMed PMID: 19218301. 
15. Van Dillen LR, Norton BJ, Sahrmann SA, Evanoff BA, Harris-Hayes M, Holtzman GW, et al. Efficacy of classification-specific treatment and adherence on outcomes in people with chronic low back pain. A one-year follow-up, prospective, randomized, controlled clinical trial. Man Ther. 2016;24:52-64. doi: 10.1016/j.math.2016.04.003. PubMed PMID: 27317505; PubMed Central PMCID: PMC4921225.

16. O'Keeffe M, Purtill H, Kennedy N, Conneely M, Hurley J, O'Sullivan P, et al. Comparative Effectiveness of Conservative Interventions for Nonspecific Chronic Spinal Pain: Physical, Behavioral/Psychologically Informed, or Combined? A Systematic Review and Meta-Analysis. J Pain. 2016;17(7):755-74. doi: 10.1016/j.jpain.2016.01.473. PubMed PMID: 26844416.

17. Beinart NA, Goodchild CE, Weinman JA, Ayis S, Godfrey EL. Individual and intervention-related factors associated with adherence to home exercise in chronic low back pain: a systematic review. Spine J. 2013;13(12):1940-50. doi: 10.1016/j.spinee.2013.08.027. PubMed PMID: 24169445.

18. McLean SM, Burton M, Bradley L, Littlewood C. Interventions for enhancing adherence with physiotherapy: a systematic review. Man Ther. 2010;15(6):514-21. doi: 10.1016/j.math.2010.05.012. PubMed PMID: 20630793.

19. Miciak M, Gross DP, Joyce A. A review of the psychotherapeutic 'common factors' model and its application in physical therapy: the need to consider general effects in physical therapy practice. Scandinavian journal of caring sciences. 2012;26(2):394-403. doi: 10.1111/j.1471-6712.2011.00923.x. PubMed PMID: 21913950.

20. O'Sullivan P, Caneiro JP, O'Keeffe M, O'Sullivan K. Unraveling the Complexity of Low Back Pain. J Orthop Sports Phys Ther. 2016;46(11):932-7. doi: 10.2519/jospt.2016.0609. PubMed PMID: 27802794.

21. Bunzli S, McEvoy S, Dankaerts W, O'Sullivan P, O'Sullivan K. Patient Perspectives on Participation in Cognitive Functional Therapy for Chronic Low Back Pain. Phys Ther. 2016;96(9):1397-407. doi: 10.2522/ptj.20140570. PubMed PMID: 27013577.

22. O'Cathain A, Thomas KJ, Drabble SJ, Rudolph A, Hewison J. What can qualitative research do for randomised controlled trials? A systematic mapping review. BMJ open. 2013;3(6). doi: 10.1136/bmjopen-2013002889. PubMed PMID: 23794542; PubMed Central PMCID: PMC3669723. 

Chapter

General Discussion 


\section{Main findings}

The findings of this thesis endorse those of previous reports, which state that exercise is a highly effective treatment to increase function and activity, as well as to decrease pain. Exercise is highly relevant to patients with subacute and chronic low back pain complaints (1-3). A recently classified subgroup of NSLBP patients has been identified, as patients with movement control impairment $(\mathrm{MCl})$. Patients within this subgroup showed significant improvements in disability and activity restriction following four to eight weeks of physiotherapeutic exercise treatment, with these improvements still remaining after one year (see Chapter 4). However, our study was unable to find significant differences between two compared exercises programs (see Chapter 3 ) - one a specific program directed at the $\mathrm{MCl}$-classified patients (4), the other, a general strengthening program. The latter had shown good results in previous studies $(5,6)$. The differences found were not significant, except the secondary outcome RMDQ, for which results showed a significant difference at the short-term follow-up. The reasons for these results (presented in Chapters 3 and 4) were: a) treatment designs were too similar, resulting in both approaches targeting the diagnosed problem; and b) other confounding influences, such as changes in lifestyle and psychosocial issues, might have diluted the treatment effect. Hence, we were unable to answer the question as to which type of exercise is most applicable to the $\mathrm{MCl}$ subgroup.

Experience gained during our RCT (e.g. relevance of chosen activities and the high number of missing values), as well as theoretical considerations, led to an additional clinimetrical analysis of the primary outcome measure used in the RCT. The Patient-Specific Functional Scale (PSFS) had previously been shown to have high responsivity in patients with moderate low back pain $(7,8)$. It measures a patient's progress, based on restrictions both relevant to and chosen by the individual himself, and which are also highly relevant to clinical practice. In Chapter 5, we analyse the psychometric properties of the PSFS and report our findings. Content validity and validity to change were inadequate when correlated to condition-specific outcome measures, such as the Roland Morris Disability Questionnaire (RMDQ). However, since the RMDQ showed similar results to the PSFS in this $\mathrm{RCT}$, the finding on the theoretical construct did not influence the actual outcome of the RCT. Thus, we conclude in Chapter 5, that the PSFS, although a clinically useful outcome measure, is difficult to apply and analyse from a research perspective.

The long term benefit of a self-directed exercise program is largely dependent on the adherence to home exercise (9). For this reason, a qualitative evaluation of strategies for, and barriers to, adherence to home exercise (Chapter 6) was embedded in the quantitative RCT. The participants in the study showed a high self-reported adherence rate in both the $\mathrm{MCl}$ and general exercise groups (10). From the patients' perspective, they gained the confidence to perform the exercises correctly during the individual therapy sessions, they attributed the improvement in their complaints to the performance of these exercises, and they integrated the exercises into a self-initiated form of regular training. The 
choice of exercises and knowledge of the diagnosis were of more minor importance. The qualitative data showed comparable patient experience in both treatment groups. Both exercise programs were equally accepted and self-initiated and adherence strategies did not differ. Of the confounding variables which could have influenced the results of the $\mathrm{RCT}$, adherence to exercise does not appear to explain the difference. The findings of this study provide further evidence on applied educational and therapeutic interventions that are already used in physiotherapy. It adds arguments in support of an individualised approach to patients in this population, rather than simply the provision of standardised information through written documents (leaflets) (11-13). In addition, it provides substantial information on improving the theoretical concepts of adherence and self-efficacy.

\section{Context of recent evidence}

\section{Classifications and building subgroups of patients with NSLBP}

Non-specific low back pain includes a wide-ranging category of patients' complaints for which no specific cause of the symptoms can be found. Radiographic findings are often suggestive of specific causes, but their correlation with symptoms are debatable (14). The state of degenerative or traumatic processes in the spine alone cannot predict the symptoms of the patient (15). However, a recent study reports that many degenerative findings in the spine region, such as disc bulging, spondylolysis and pathological changes in the vertebrae, have a higher prevalence in individuals 50 years of age or younger with self-reported low back pain, compared to asymptomatic individuals (16). Moreover, the authors point out that although these radiographic findings may be used as biomarkers for further research, they do not prove that degeneration causes LBP. The non-specific aspect of pain in this population often leads to untargeted treatments, such as ineffective surgery, medication and exercises. Therefore, classifications, as introduced in Chapter 1 , may be the key to a more targeted physiotherapy treatment.

A recent review confirmed the positive effects of exercise on patients with NSLBP (17). It also evaluated which kind of exercises had a beneficial effect on pain for patients with chronic NSLBP when compared with non-exercise treatments. The 39 studies included in the review analysed different treatment groups and the results showed a small, but significant, positive observed effect for strength/resistance exercises and coordination/stabilisation exercises. Viewed in this context, it is clear that we used the most promising exercise interventions in our RCT for a defined target subgroup of NSLBP patients with $\mathrm{MCl}$. Although our results confirm the findings of the mentioned review, that the two exercise procedures are effective, we were unable to identify additional benefits from either one of the two recommended exercise treatments. 
Karayannis et al. (18) selected the five most recognised classifications of NSLBP with mainly biomechanical aspects: Mechanical Diagnosis and Therapy (MDT), Pathoanatomical Classifications (PAC), Treatment Based Classifications (TBC), Movement System Impairment (MSI), O'Sullivan Classifications (OSC); and analysed each representation during a clinical examination. We have previously made a brief introduction to four of these subgroups (Chapter 1). TBC, together with PAC and MDT, follow a loading strategy with repeated movements to the spine. MSI and OSC follow a movement modification strategy, as described extensively in this thesis. The assessment and treatment of NSLBP by means of movements and postural changes is common to all five classifications and treatments in this study. One experienced physiotherapist examined and classified 102 patients into one or more classification systems. They found overlap and discordance between the classifications. It was suggested that an integrated assessment could widen intervention options. In our trial, we attempted to fill this gap between classifications, with a combination of tests which allowed the definition of a subgroup with $\mathrm{MCl}$. Furthermore, we integrated several treatment approaches for these $\mathrm{MCl}$ patients. We initially included patients who presented with pain behaviour related to movement and body positions - a behaviour which is included in all classifications. Secondly, as further inclusion criteria, patients had to fulfil minimum requirements, as tested with the $\mathrm{MCl}$ test battery. This includes selected tests described in MSI. And thirdly, the sub-classification of $\mathrm{MCl}$ for the specific treatment was based on the principles of the O'Sullivan classification system. However, based on our results, drawing conclusions on exercise interventions from this new assessment is not possible.

A 2016 published Cochrane Review analysed 29 articles focused on stabilisation exercises, including restoration of specific muscle activity (i.e. m. transversus abdominis, mm. multifidi) and active training, which they called 'motor control exercise (MCE) (19). Our RCT was excluded from this review because we did not follow the initial muscle reactivation concept; in addition, both of our two interventions included aspects of MCE, such as strengthening exercises. Nevertheless, the findings of the review clearly confirmed the results of our RCT. For disability, they reported that there is a small, but clinically unimportant, effect of MCE exercises in the short term but no effect at the long-term followup, when compared with other exercises. Furthermore, the authors of the review noted that future studies should include adequate sample sizes, long-term follow-ups and further subgrouping; all these suggestions have been fulfilled in our study.

It can be concluded, that all these recent studies on NSLBP included the recommendation to classify subgroups with the objective that future studies should identify more targeted exercise treatments. The question remains unanswered as to which exercises are most relevant for which subgroup. Based on our clinical experience and our experiences during the RCT, we see two directions for future research projects concerning subgroups. Firstly, we conclude that psychosocial factors, such as behavioural aspects, or the therapist/patient relationship, may have influenced our results more than the purely mechanical exercise protocol. Future trials should place focus on these factors. Secondly, 
our trial was not designed to investigate the further sub-classification (flexion, extension, unilateral impairment) of $\mathrm{MCl}$. However, for clinical practice it is important to know whether the further differentiation of the subgroup would bring benefits, due to even more targeted specific exercises. Future research projects should gain insight into the effects, differences and difficulties of applying specific and individually-tailored exercises to even smaller, differentiated subgroups. At this individual level research could require alternative research designs, such as replicated single case studies.

\section{Subgroup-specific exercise studies}

As described above, NSLBP patients differ in presentation and disability. For this reason, subgrouping and the subsequent analysis of these differentiated subgroups is proposed.

The identified subgroup of $\mathrm{MCl}$ studied in our RCT included patients with specific complaints (pain in static positions) and aberrant movements, assessed using an $\mathrm{MCl}$ test battery ( 2 positive tests out of a set of 6 tests). The main contrast between treatments was the approach of specific-exercises focused on improving these impaired movements, versus a non-specific generic strengthening approach.

It is interesting to note, that there is one study that followed the same treatment protocol for patients with subacute $\operatorname{NSLBP}(20,21)$. In this study and our RCT, the patients in both treatment groups improved to a higher level of functioning, with this improvement being maintained for over one year. These results underline the importance of exercise for this $\mathrm{MCl}$ subgroup of patients. However, the other research group reported a significantly greater reduction in disability and pain due to the specific exercises versus general exercises, both in the short term and at the one-year follow-up. This difference might be explained by the following divergences between protocols: our study excluded patients with high psychosocial risk, while the other study excluded patients with motor learning difficulties; we included chronic patients, while they excluded them; we used a multicentre design, while they used a single location; finally, different statistical analysis was performed and the choice of primary outcome was different (PSFS vs. RMDQ).

A recently reported $\mathrm{RCT}$, following a protocol with similarities to this $\mathrm{RCT}$, examined the effect of specifically-tailored exercises compared with deadlift exercises on patients with $\operatorname{NSLBP}(22,23)$. Patients were included when they demonstrated pain during certain movements. The specific intervention applied was aimed at the individual movements reported as painful. The exercises were specifically selected and designed to normalise the movement impairment for each patient. Patients in the control group performed deadlifts under therapeutic supervision. This was to ensure that the spine was in the neutral position, the appropriate weight was chosen and to advise on the rate (slow) of progression. Unlike our RCT, no subgroup was formed prior to inclusion and no sub-classification determined the individual treatment. The first published study of this research group used the PSFS as the primary outcome. It reported a significant inter-group difference in activity restriction in favour of specific exercises, both in the short term and long 
term (1 year). Additionally, the participants in this group showed significant improvement on the movement control test battery, which was selected as the secondary outcome (24). No differences were found for pain or other physical measures. The second study of this research group, using the same exercise programs as in their first study, revealed no differences in disability (Roland Morris), pain intensity and health-related quality of life, either in the short term or at the 1 and 2 year follow-ups. The first study was similar to our RCT in the choice of PSFS as the primary outcome and the use of the test battery. However, with our reservations towards PSFS, which we have explained in Chapter 5, we should treat the results with caution. This would be even more the case where the PSFS (activities with difficulties) and physical examination (movements with difficulties) and specific treatment (exercise for movement difficulties) are too related. In this case the results would represent the quality of treatment rather than any difference in treatment effects. The fact that PSFS and the test battery improved simultaneously may imply that the specific treatment and the two tests belong to the same construct. We did not find the same association. With regard to the second study, the results endorsed those of our study. An advantage in the design of the two described studies was their choice of treatment alternatives, which were distinctively different from each other but well recognised in rehabilitation therapy. However, neither of the studies of this research group assessed the participants for a classification into subgroups. The most positive conclusion that can be made is that patients with NSLBP respond better to low-load than to high-load exercise. For future projects, we should also consider that subgrouping may be of less importance than a clear contrast between the types of exercises.

Van Dillan et al. (2016) reported results of an RCT which treated NSLBP with exercise treatment, according to the classification of Movement System Impairment (MSI) described by Sahrman. This subgroup is similar to our $\mathrm{MCl}$, but includes additional biomechanical aspects, such as combined impaired movement varieties. In this trial, a specific impairment-driven exercise program was compared to a general-exercise program, similar to the one in our trial. They also found no differences in pain or disability in the short or long term. Interestingly, both treatment groups received instructions on how to integrate their movements into daily activities in addition to their exercise training; these they refer to as performance training. This performance training was classification-specific or generic, according to the treatment alternative. The authors found that, irrespective of the treatment group, adherence to the performance training correlated with the Oswestry Disability Index. They discussed, firstly, whether adherence to performance is more important than adherence to exercise and, secondly, whether adherence to performance is related to the good improvement in disability seen in both groups. According to the protocol of our RCT, only the experimental group received advice on how to include the new movements into daily life. However, in our qualitative analysis, patients in both groups commented on this issue. We cannot exclude that in our pragmatic, multicentre trial that some sort of advice was given to all participants, but to distinguish between 
adherence to exercise and adherence to performance may be an interesting element for future research.

To conclude this paragraph, out of the large number of 'stabilisation' exercises, the most recent studies confirm the findings of the 2016 Cochrane Review (19, 25). Additionally, the inter-group differences may be larger for patients in a subacute state than those in a chronic state. Current literature supports the idea that patients with impaired movements become a significant benefit from performance of exercise. The benefits of forming a subgroup are still inconclusive, whether it is on the basis of a test battery or on an individual physical diagnosis. Some authors prefer a multidimensional analysis (adding psychological, social, lifestyle, comorbid health or non-modifiable factors), especially for patients with chronic LBP (26). The individual physical exercise treatment would then be complemented with individual behavioural, educational or interpersonal aspects. However, whether this would add a (cost efficient) benefit remains to be evaluated. The question, "which exercise is best for which subgroup" has still not been answered conclusively.

\section{Specific exercises combined with technical support}

Technical devices have been developed in recent years to improve subgroup-specific assessment, monitor outcomes and enhance treatment.

The subgroup identification in our trial relies on assessment by therapist observation. Although our test battery has shown good reliability in previous studies (24), today we can evaluate the validity of the test battery with the aid of technical support. Likewise, treatment progress following exercise training can be monitored, and even improved, through technical assistance. At the time of designing our trial the technical devices described below were still in a state of development. These devices could have an impact on future assessments and therapy procedures and may provide insight into the underlying mechanisms. Therefore, they are introduced below.

The evaluation of movement assessment (used in the clinic and research) by means of wireless movement analysis systems using body-worn sensors (e.g. Valedo ${ }^{\circledR}$ from Hocoma AG, ViMove from dorsaVi) is proving most promising.

Bauer et al. developed and analysed the Valedo ${ }^{\circledR}$ system, which measures trunk movements with a wireless inertial measurement device using surface sensors (27-29). In a comparison, they reported that the system is a valid alternative to an optoelectronic camera system (video-based measures) and allows monitoring of longitudinal results of movement performance. Secondly, with a multiple testing design, they used the system to analyse the reliability of the $\mathrm{MCl}$ test battery in healthy persons and patients complaining of chronic LBP. The results regarding the various $\mathrm{MCl}$ tests varied. While the reliability tests for 'waiters bow', 'pelvic tilt' and 'prone knee bend' showed high reliability, those for 'sitting knee extension', 'rocking backwards' and 'rocking forwards' showed low-tomoderate reliability. The results contradicted the previous observation-based interrater reliability results, which had a moderate-to-excellent value (24). The authors commented 
that a comparison may be difficult due to different quantification approaches. The same research group further utilised the Valedo ${ }^{\circledR}$ system to assess movement patterns in participants with different pain levels (NRS 0-7)(28). They found no significant effect of pain on the performance of two selected $\mathrm{MCl}$ tests ('waiter's bow', 'sitting knee extension'). However, they found greater variability of movement patterns in LBP participants when repetitively picking up a box at a predetermined, fast speed. The authors conclude that there is a connection between pain and altered movement patterns, even though they could not link the two $\mathrm{MCl}$ tests to pain levels. The study reveals that pain and uncontrolled movements coexist, although cause and effect are still unsolved.

In hindsight, our RCT could have benefitted from more accurate assessment and subsequent classification supported by technical devices, provided the devices had delivered valid and reliable measures. This could have had an effect on study inclusion (2/6 tests positive) and on sub-classification, as well as the specific treatment. The more accurate the assessment, the more target-specific the treatment can be. Future research to validate the remaining $\mathrm{MCl}$ tests, or even the combination of several tests as we performed in the test battery, may bring further insight into our assessment procedures.

The application of technical devices to physiotherapy treatment was reported in a pilot study by Kent et al. (30). In patients with subacute and chronic LBP, this study compared a movement-specific individually-targeted exercise treatment supported by a portable biofeedback device (ViMove) to a guideline-oriented treatment accompanied by a sham device. The ViMove device was used to analyse movement patterns during the assessment, to collect movement data and data concerning movement changes, as well as to provide patient-specific feedback alerts by audio and vibration function during activities of daily living. Data was collected over a period of ten weeks, with a follow-up after one year. The outcomes for disability (Roland-Morris), activity limitation (PSFS) and pain (VAS) significantly favoured the biofeedback group in all inter-group comparisons, including the rate of change over time. The authors concluded that, based on their results, movement training combined with biofeedback can result in long-lasting improvements of the measured outcomes and that future fully powered studies using the biofeedback system are justified. The main focus of this study was to investigate the application of the new technology and was not designed primarily to compare the two treatment alternatives. It remains to be seen whether the added biofeedback system provides additional benefits when the exercise treatment is identical. However, the fact that the same outcomes as in our study differed so much with the use of a biofeedback system is surprising. It supports our suggestion that not only cognitive factors and adherence to exercise, but also changes in the activities of daily living, may result in the long sought-after effect (Chapter 6). The introduction of a portable biofeedback could be a valuable treatment addition for patients with $\mathrm{MCl}$.

Other electronic assessments, such as electromyography (EMG), centre of pressure (COP) on a force platform and video-based analysis of kinematics, have been applied as outcome measures after exercise therapy. 
Lomond et al. $(31,32)$ applied surface EMG to evaluate muscle activity in the legs and trunk during voluntarily lifting of one leg in the lying supine position. Participants with LBP $(n=15)$ showed an earlier and higher amplitude activation in most muscles than participants without LBP $(n=15)$. The same measurement protocol revealed no normalisation of the activation pattern after 6 weeks of exercise treatment in patients with chronic LBP ( $\geq 12$ months). The study compared MCE stabilisation exercises $(n=12)$ (motor control exercise, deep abdominal, strengthening trunk, educational booklet) with movement system impairment ( $n=21)$ (MSI: modifies trunk movements and postures relevant to patients' symptoms). Both groups improved significantly following treatment, with no difference between the groups for function (measured using the Modified Oswestry Disability Index (ODI)) or pain (Numeric Pain Rating (NPR)).

The same research group compared the effects of MCE treatment $(n=29)$ with that of general strengthening exercises ( $n=29$ ) on an EMG of trunk and leg muscles (31). For this study, patients with chronic NSLBP stood on a perturbed surface. In order to assess postural adjustments, they also analysed the centre of pressure (COP) displacement during platform perturbations. Function (ODI) and pain (NPR) improved significantly with no inter-group differences after 11 weeks and 6 months. However, baseline and activation amplitude of the EMG responded differently to the treatments. Patients showed an increased baseline abdominal activity after MCE treatment and an increase in baseline activity of all muscles in the control group. Both showed a decrease in activation incidence in the post-perturbation phase. The authors point out that neither treatment promotes an automatic, healthy postural response, since both treatments promote stiff and rigid movement behaviour when automatic muscle reactions are preferred.

This insight derived from the EMG results raises the question as to when and whether our treatment strategies to encourage motor/movement control of the lumbar spine under static and dynamic conditions are indicated. Firstly, from a clinical point of view, the short-term results of Lomond's study for the baseline muscle activation patterns of either treatment are not surprising. This reaction is strived for in clinical practice to prevent tissue overload, with the objective, however, that the altered behaviour becomes automatic and leads to normalised long-term muscular activity. Secondly, the protocol of both experiments allowed practise of the measurement procedures. This allows an anticipation and, subsequently, early muscular activation. In addition, it has been suggested that this stiffening is a protective behaviour driven by pain and experience $(33,34)$. In consequence, this process could promote potential negative effects, such as reduced movement and variability of movements. Based on the fact that all treatments benefitted the patients in terms of function and pain, the unchanged, or even supposed disadvantageous, muscular effects remain unclear. We must consider that the progression of the exercise strategies should be varied according to the healing phase, behavioural progress and personal needs. This reasoning supports the formation of subgroups. The $\mathrm{MCl}$ treatment in our RCT is based on specific sub-classification and treatment. 
Recently, one study employed a $\mathrm{MCl}$ sub-classification $(\mathrm{n}=32)$ of patients with pain in lumbar extension and increased lordosis to test the effect of MCE versus an $\mathrm{MCI}$ program. Outcome variables ODI and NRS showed an improvement in function and pain for both treatments, with no significant difference between the groups after 4 weeks of treatment (35). Using EMG, a flexion relaxation rate was measured on the lumbar extensor muscles during full flexion in standing. The $\mathrm{MCl}$ treatment showed a partial normalisation of the relaxation pattern, especially in the thoracic part of the lumbar iliocostalis. This may be a first step towards using EMG on a subgroup level. However, we must take into account that relaxation of the extensors is part of a specific $\mathrm{MCl}$ training and the results support only the validity of the treatment.

$\mathrm{Ng}$ et al. measured regional lumbar kinematics during sitting and during 15 minutes of rowing on an ergometer using a video-based laboratory system (36). This measurement served as a secondary outcome and followed after 8 weeks of treatment in adolescent male rowers suffering from LBP related to rowing. The study compared an individually-targeted, cognitive, functional treatment (based on the O'Sullivan classification, treated with movement control plus cognitive treatment approach), with a control group who were free to seek treatment from healthcare providers external to the project. The intervention group reported significantly less pain during rowing (NRS), disability (RMDQ) and activity restriction (PSFS) when compared to the control group. The improvement remained for 12 weeks. The video-system measured an increased angle in the lower lumbar region during static sitting in the intervention group; however, it found no significant difference in the kinematic measurements during rowing between the groups. The authors suggested that factors other than kinematics were responsible for the positive effect on pain. They proposed that the cognitive approach positively influenced perception in daily life and the pain tolerance during rowing. This study supports the previously described model, that not only mechanical causes are responsible for the development of pain (37). Including the behavioural aspects of daily life into our treatment procedures and addressing the biopsychosocial influencers, may affect pain perception, even during mechanically challenging tasks; this could be a valuable addition to our $\mathrm{MCl}$ treatments used in the RCT.

The application of the described technical devices in the clinical care of patients with LBP is a potential positive addition. It cannot replace individual assessment and treatment, but it may support the clinician through providing additional information. However, their usage comes with some constraints: clinicians need to be trained in the use of the systems; all systems are associated with considerable costs; and they are often location dependent. Nevertheless, further developments in portable, wireless devices for home-based use as biofeedback would be of value.

In the context of research in the field of $\mathrm{MCl}$, technical support could enhance the quality of a research protocol. Inertial wireless devices, especially, could increase the validity of inclusion criteria, classification and sub-classification and, at best, measure outcomes. All the described devices have added insight into the mechanisms behind $\mathrm{MCl}$. It 
remains to be seen whether these devices combined with new developments, such as brain scans and behavioural assessments, will lead to further models of the mechanisms behind exercise for $\mathrm{MCl}$. $(38,39)$.

\section{Adherence to exercise}

Adherence is generally defined as "the extent to which a person's behaviour ...corresponds with agreed recommendations from a health care provider" (40). Non-adherence to exercise may have a negative impact on treatment effects $(41,42)$. There is moderate evidence that strategies to improve adherence to exercise have a positive effect on pain and function (9). Adherence to exercise in patients with low back pain is reported to be low, with some 50 to $70 \%$ of patients non-adherent to prescribed exercises (43). In our study, it was essential to monitor the adherence level for the two treatment alternatives, since it could have had a substantial effect on outcomes.

According to recent literature, the lack of adequate tools to measure adherence is a major hindrance to its evaluation. In a review by Hall et al. in 2015, it was reported that no studies assessing adherence used the same assessment tool (44). The identified tools varied in their format (diary, single question, or multi-item questionnaire), specific question(s) and methods of scoring. The results were confirmed by an extended review in 2017, which pointed out that of seven clearly described and reproducible tools, all had essential inadequacies (42).

Self-reported adherence to exercise measured with single questions, which was used in our RCT, should be regarded with caution because of detection bias ( systematic differences between groups in how outcomes are determined) (45). We asked "How often were you able to do your exercises which were recommended in the physiotherapy", and allowed categorical answers, "daily, 2x per week, rarely, never". Twice per week was the recommended frequency. We reported $46 \%$ (42 patients) in the trial who still did their exercises twice per week or more after one year and regarded the adherence as high in relation to previous studies (Chapter 3 ). We found no differences between treatment groups and concluded that adherence was not confounding our outcomes.

With the additional qualitative analysis, we learned more about the strategies to remain adherent to exercise. Even if we acknowledge an overestimation due to self-reporting, the adherence rate found in our RCT was relatively high. This result can be explained by inclusion of the coaching strategy to improve confidence and self-efficacy, as presented in Chapter 6.

Self-efficacy may have a major impact on outcomes in the treatment of low back pain $(46,47)$. Self-efficacy describes a person's own belief that he/she can successfully execute a program to meet a desired outcome (48). Improvement of self-efficacy is of importance in clinical practice (42). The new findings, described in Chapter 6, where patients regarded self-initiated training as a helpful tool for adherence, may add evidence to support this treatment concept. 
Additional support to our findings from the qualitative study comes from the most recent literature review, which listed the factors that influence adherence to home-based exercise for all diseases (49). The barriers listed, amongst others, were low social support, lack of adequate feedback, large number of exercises, low self-motivation, low self-efficacy, lack of time, previous non-adherent behaviour and worsening of pain. All these factors were discussed in our patient groups with low back pain. We can conclude that our participants may have had a higher self-motivation and efficacy simply from the fact that they had chosen to participate in a long-lasting exercise study. Additionally, they were screened for their psychosocial status prior to inclusion, which had an influence on the selection. Nevertheless, the new insights from our qualitative study, explaining how to maintain motivation and efficacy, are valuable additions to our knowledge and have clinical implications.

Furthermore, as described above (subgroup-specific paragraph), future study designs to evaluate adherence to exercise should pay special attention to the rate of exercise performance (acceptance of how often and how long the exercises should be performed), adherence to performance in daily life (acceptance of advice on how to integrate exercise into daily life) and adherence to lifestyle changes in general (acceptance of behavioural changes).

\section{Methodological reflection}

In our reflection on the methodological issues of our randomised controlled trial, we should first mention that we followed the CONSORT statement when reporting our study design and data (50). The RCT was designed according to quality standards to reduce the risk of bias (51). As it is essentially a pragmatic design, we could not blind therapists and patients to the intervention.

During the trial design, we anticipated insufficient recruitment of patients because, as a teaching and research institute, we did not have direct access to patients. For recruitment, we chose to contact eight private practices and five hospital outpatient departments in three cantons in Switzerland. Logistically, this was followed by three different ethics applications and procedures, instruction of assessment and treatment to personnel in each facility, and the formation of a central administration office. Despite these actions, recruitment remained slow and we decided to send out an electronic advertisement to staff and students of the ZHAW. We also instructed additional physiotherapists who provided in-house treatments to 30 participants. The ZHAW participants had to fulfil the same inclusion criteria, as described in the protocol, and followed the same randomisation and treatment procedures. Through all these efforts, we managed to reach the calculated number of 106 included patients. The connection with the ZHAW may have influenced the social distribution and the voluntary step to get in contact (rather than 
being recommended by a doctor) shows a pre-existing self-motivation. However, no influence of the recruitment procedure on the final results was found.

The multicentre design of the study also implied that we had to expect location-specific factors which could potentially influence results. For example, recruitment could have been influenced by logistical problems of the patients and the clinics (selection bias). Although we provided training for the physiotherapists, there was no detailed comparison of the treatment procedures. All these factors were controlled through randomisation of the patients.

The expected variability of the patient group itself needs attention during the design of an RCT. These variables were assumed to be equally distributed across both treatment groups due to the randomisation procedure. Regression analysis showed no effects of these variables on outcome. However, for logistical reasons we decided not to assess for quality of life, medication or behavioural strategies (to name just a few) which could have had an influence on our results. Results of a well-controlled RCT are rated high in evidence, but do not give much insight into the influence of confounders. Alternative designs, such as replicated single case studies, may give more in-depth interpretation of the data by directly reflecting what is important to individual patients. Including $\mathrm{MCl}$ patients with different sub-classifications of replications may add clinically valuable information and eventually achieve generalizable results.

The choice of the relevant primary outcome was given considerable thought in the design phase. The choice of the outcome PSFS, as opposed to the RMDQ, was based on study results at the time and on clinical decision-making. The PSFS had been reported as being more responsive to moderate pain situations, which was the expectation in our patient group (8). It also reflected the reporting in clinical practice of patients' signs and symptoms. In hindsight, and with the knowledge of our results (Chapter 5), we consider that other outcomes, such as the RMDQ or ODI for disability, would have been more reflective of long-term improvement.

The limited contrast between treatment arms transpired to be a major problem in the design of the study. From a clinical point of view, the choice of exercises based on subgrouping plus additional classification, represents a distinctly tailored treatment program. A generic exercise program, which has been shown to be effective, represents a credible control group. Our interpretation of the quantitative long-term data concludes that, given the many influencing factors over the course of one year, the contrast between the exercise programs was not sufficient to produce a difference. Moreover, while the two treatment programs were initially very different ( $\mathrm{MCl}$-control movements; $\mathrm{GE}-$ generic exercises), during the progression of the $\mathrm{MCl}$ group, once control was reached, the training included similar exercises.

A long-term follow-up after one year is important considering the long-term course of subacute and chronic pain. The expected difficulty in this RCT was long-term accessibility to the patient. For primary and secondary outcome measures, using direct telephone contact and postal questionnaires, we reached a very good rate of access. Only four patients 
did not respond. But it was not feasible (time, finances, independent assessors) to invite all participants for physical examinations at the long-term follow-up. With the results of the $\mathrm{MCl}$ test battery, we could have made further conclusions regarding the subgroup. In conclusion, the logistics and costs of an RCT are extremely high.

Embedding qualitative research into an RCT adds considerable evidence to the effectiveness of health interventions (52). The qualitative study presented in Chapter 6 provides a means of interpreting the RCT results and describes contextual features of potential use in exercise treatments and future trials.

The qualitative evaluation revealed that patients were equally supported by the programs in both groups; they increased their general physical activity and performance in daily living. The importance of a patient-perceived individual treatment and a self-driven long-term strategy may have had a higher impact on the results than the choice of exercises. Clinicians' decisions on individual exercises are dependent on professional experience and patients' perception and/or acceptance. These requirements are major motivational factors to the patient.

From a research perspective, we can say that patients in the subgroup $\mathrm{MCl}$ will have a high chance of improvement over the short and longer term through continued exercise. This statement is particularly important in NSLBP, since it is initially a relatively shortterm problem, but needs special management to avoid/treat a longer term problem. Further exploration of these factors, which became apparent in the qualitative part of this thesis, may eventually lead to symptom-effective and cost-effective treatments.

\section{Theoretical implications}

The concept of assessment and treatment of movement control impairment needs a critical reconsideration. Assessment of aberrant movements in patients with low back pain, as applied in this RCT, is clinically very useful. The test battery is easily applied by both novice and expert physiotherapists. However, not all sub-classifications (i.e. flexion, extension, unilateral, multidirectional) of the $\mathrm{MCl}$ group can be reliably detected with this battery. Additionally, treatment of all possible combinations of sub-classifications may need an even higher variation of specific-exercises than allowed for in the experimental protocol of this RCT. Multidirectional impairments, especially, may need a long-term adjustment of the exercises. The treatment described in the protocol may still not be specific enough to produce differences in outcomes. Limitation of the RCT inclusion criteria to the most common sub-classifications (flexion, extension) may result in the selection of an even more specific subgroup. Exercises could then address $\mathrm{MCl}$ signs in a standardised manner during treatment. Although it would make standardisation of treatment easier, it would come at the cost of even greater recruitment problems for a fully powered RCT. A possible solution may be the alternative research methods discussed above. 
Our studies could not prove a connection between the $\mathrm{MCl}$ subgroup specific-exercises and the outcomes of function, activity and pain. Only if the $\mathrm{MCl}$ specific treatment had resulted in a benefit, could we have concluded that aberrant movements had a direct connection to these outcomes. This connection could be a cause or effect of NSLBP. All we can say in this study is that the $\mathrm{MCl}$ subgroup was certainly most receptive to exercise treatment, whether it was movement-specific exercises or non-specific strength exercises. In the large group of subacute and chronic NSLBP patients this classification is highly valuable.

\section{What this research adds}

The studies presented in this thesis have added knowledge to the field of exercise treatment for patients with low back pain. The subgroup of patients presenting with movement control impairment had excellent outcomes when treated with exercise.

Our results do not indicate a preference for any specific type of exercise for this subgroup; it still relies on the individual decision of the therapist as to which exercise is most applicable. We were able to show that exercise, supported by consistent coaching of the patient, achieves long-term, lasting beneficial effects on function, activity and pain. This in turn improves the confidence and self-efficacy of the patient and should be included in clinical treatments. In our analysis of the outcome measure (PSFS), we critically questioned the application of the measure for long-term data sets.

\section{Recommendations for clinical practice}

Exercises are highly beneficial to patients classified into one of the $\mathrm{MCl}$ subcategories. Over the long term the positive effects are maintained. The choice between specific $\mathrm{MCl}$ exercises and generic strengthening exercises remains unclear. Patients value a correct instruction and supervision higher than the choice of exercise. For clinical practice, we can recommend an assessment of $\mathrm{MCl}$. However it may be more beneficial for treatment if the therapist chooses exercises which, firstly, he/she is personally best at instructing and, secondly, which he/she tailors to the individual needs of the patient.

Long-term adherence to a recommended exercise program is increased through patient education and coaching. The main motivator is the perceived direct effect between exercise and pain by the patient, as well as the individual approach. Information on diagnosis and theory may be introduced for interested patients. Coaching for long-term adherence to the recommended exercises should include a positive binding to self-initiated physical activities on an individual basis.

The patient specific functional scale (PSFS) is a useful tool for short-term evaluation. Impaired activities change during long-term treatments. Therefore, data on the activities 
must be adjusted. This comes with the cost that long-term analysis using this measure is fraught with complications.

\section{Recommendations for future research}

The RCT of our group, as well as recent publications investigating exercise treatment subgroups, have found no clear beneficial treatment procedures. To date, the consistent positive results cannot be explained. Based on this thesis, we recommend the following research: firstly, a further exploration of the sub-classifications of $\mathrm{MCl}$, which may reveal more detailed results. Alternative study designs, such as replicated single case studies, which may provide insights based on individual patient patterns. Secondly, we strongly recommend a comparison of treatments with sufficient contrast in future experiments, since there is a strong tendency in the case of exercise programs to overlap treatment aims and performance of movements. Thirdly, exercise in combination with non-mechanical influences, such as psychological and social factors, should be explored. Lastly, the effects of patient education and exercise adherence, as explained above, need further investigation. 


\section{References}

1. Airaksinen O, Brox JI, Cedraschi C, Hildebrandt J, Klaber-Moffett J, Kovacs F, et al. Chapter 4. European guidelines for the management of chronic nonspecific low back pain. Eur Spine J. 2006;15 Suppl 2:S192300. doi: 10.1007/s00586-006-1072-1. PubMed PMID: 16550448; PubMed Central PMCID: PMC3454542.

2. Hayden JA, van Tulder MW, Malmivaara A, Koes BW. Exercise therapy for treatment of non-specific low back pain. Cochrane Database Syst Rev. 2005(3):CD000335. Epub 2005/07/22. doi: 10.1002/14651858. CD000335.pub2. PubMed PMID: 16034851.

3. Liddle SD, Baxter GD, Gracey JH. Exercise and chronic low back pain: what works? Pain. 2004;107(1-2):17690. PubMed PMID: 14715404.

4. Saner J, Sieben JM, Kool J, Luomajoki H, Bastiaenen $\mathrm{CH}$, de Bie RA. A tailored exercise program versus general exercise for a subgroup of patients with low back pain and movement control impairment: Shortterm results of a randomised controlled trial. J Bodyw Mov Ther. 2016;20(1):189-202. doi: 10.1016/j.jbmt.2015.08.001. PubMed PMID: 26891655.

5. Luomajoki H, Kool J, de Bruin ED, Airaksinen O. Improvement in low back movement control, decreased pain and disability, resulting from specific exercise intervention. Sports Med Arthrosc Rehabil Ther Technol. 2010;2:11. Epub 2010/04/27. doi: 1758-2555-2-11 [pii] 10.1186/1758-2555-2-11. PubMed PMID: $20416091 ;$ PubMed Central PMCID: PMC2873574.

6. Luomajoki H. Movement Control Impairment as a subgroup of non-specific low back pain. Institute of Clinical Medicine, School of Medicine, Faculty of Health Sciences. 22.10.2010 ed. Kuopio: Eastern Finland University Library/Sales of Publications; 2010. p. 70.

7. Pengel LH, Refshauge KM, Maher CG. Responsiveness of pain, disability, and physical impairment outcomes in patients with low back pain. Spine (Phila Pa 1976). 2004;29(8):879-83. Epub 2004/04/15. doi: 00007632-200404150-00011 [pii]. PubMed PMID: 15082988.

8. Hall AM, Maher CG, Latimer J, Ferreira ML, Costa LO. The patient-specific functional scale is more responsive than the Roland Morris disability questionnaire when activity limitation is low. Eur Spine J. 2011;20(1):79-86. doi: 10.1007/s00586-010-1521-8. PubMed PMID: 20628767; PubMed Central PMCID: PMC3036014.

9. Jordan JL, Holden MA, Mason EE, Foster NE. Interventions to improve adherence to exercise for chronic musculoskeletal pain in adults. Cochrane Database Syst Rev. 2010(1):CD005956. doi: 10.1002/14651858. CD005956.pub2. PubMed PMID: 20091582.

10. Saner J, Kool J, Sieben JM, Luomajoki H, Bastiaenen $\mathrm{CH}$, de Bie RA. A tailored exercise program versus general exercise for a subgroup of patients with low back pain and movement control impairment: A randomised controlled trial with one-year follow-up. Man Ther. 2015;20(5):672-9. doi: 10.1016/j.math. 2015.02.005. PubMed PMID: 25770419.

11. Coudeyre E, Tubach F, Rannou F, Baron G, Coriat F, Brin S, et al. Effect of a simple information booklet on pain persistence after an acute episode of low back pain: a non-randomized trial in a primary care setting. PloS one. 2007;2(8):e706. doi: 10.1371/journal.pone.0000706. PubMed PMID: 17684553; PubMed Central PMCID: PMC1939729.

12. Coudeyre E, Givron P, Vanbiervliet W, Benaim C, Herisson C, Pelissier J, et al. [The role of an information booklet or oral information about back pain in reducing disability and fear-avoidance beliefs among patients with subacute and chronic low back pain. A randomized controlled trial in a rehabilitation unit]. Annales de readaptation et de medecine physique : revue scientifique de la Societe francaise de reeducation fonctionnelle de readaptation et de medecine physique. 2006;49(8):600-8. doi: 10.1016/j.annrmp.2006.05.003. PubMed PMID: 16793163.

13. Cherkin DC, Deyo RA, Battie M, Street J, Barlow W. A comparison of physical therapy, chiropractic manipulation, and provision of an educational booklet for the treatment of patients with low back pain. $\mathrm{N}$ Engl J Med. 1998;339(15):1021-9. doi: 10.1056/NEJM199810083391502. PubMed PMID: 9761803.

14. Balague F, Mannion AF, Pellise F, Cedraschi C. Non-specific low back pain. Lancet. 2012;379(9814):482-91. doi: 10.1016/S0140-6736(11)60610-7. PubMed PMID: 21982256. 
15. Steffens D, Hancock MJ, Maher CG, Williams C, Jensen TS, Latimer J. Does magnetic resonance imaging predict future low back pain? A systematic review. Eur J Pain. 2014;18(6):755-65. doi: 10.1002/j.15322149.2013.00427.x. PubMed PMID: 24276945.

16. Brinjikji W, Diehn FE, Jarvik JG, Carr CM, Kallmes DF, Murad MH, et al. MRI Findings of Disc Degeneration are More Prevalent in Adults with Low Back Pain than in Asymptomatic Controls: A Systematic Review and Meta-Analysis. American Journal of Neuroradiology. 2015;36(12):2394-9. doi: 10.3174/ajnr.A4498.

17. Searle A, Spink M, Ho A, Chuter V. Exercise interventions for the treatment of chronic low back pain: a systematic review and meta-analysis of randomised controlled trials. Clinical rehabilitation. 2015;29(12):1155-67. doi: 10.1177/0269215515570379. PubMed PMID: 25681408.

18. Karayannis NV, Jull GA, Hodges PW. Movement-based subgrouping in low back pain: synergy and divergence in approaches. Physiotherapy. 2016;102(2):159-69. doi: 10.1016/j.physio.2015.04.005. PubMed PMID: 26126426.

19. Saragiotto BT, Maher CG, Yamato TP, Costa LO, Costa LC, Ostelo RW, et al. Motor Control Exercise for Nonspecific Low Back Pain: A Cochrane Review. Spine (Phila Pa 1976). 2016;41(16):1284-95. doi: 10.1097/BRS.0000000000001645. PubMed PMID: 27128390.

20. Lehtola V, Luomajoki H, Leinonen V, Gibbons S, Airaksinen O. Sub-classification based specific movement control exercises are superior to general exercise in sub-acute low back pain when both are combined with manual therapy: A randomized controlled trial. BMC Musculoskelet Disord. 2016;17:135. doi: 10.1186/s12891-016-0986-y. PubMed PMID: 27005470; PubMed Central PMCID: PMC4804617.

21. Lehtola V, Luomajoki $H$, Leinonen V, Gibbons S, Airaksinen O. Efficacy of movement control exercises versus general exercises on recurrent sub-acute nonspecific low back pain in a sub-group of patients with movement control dysfunction. Protocol of a randomized controlled trial. BMC Musculoskelet Disord. 2012;13:55. doi: 10.1186/1471-2474-13-55. PubMed PMID: 22494776; PubMed Central PMCID: PMC3353835.

22. Aasa B, Berglund L, Michaelson P, Aasa U. Individualized low-load motor control exercises and education versus a high-load lifting exercise and education to improve activity, pain intensity, and physical performance in patients with low back pain: a randomized controlled trial. J Orthop Sports Phys Ther. 2015;45(2):77-85, B1-4. doi: 10.2519/jospt.2015.5021. PubMed PMID: 25641309.

23. Michaelson P, Holmberg D, Aasa B, Aasa U. High load lifting exercise and low load motor control exercises as interventions for patients with mechanical low back pain: A randomized controlled trial with 24-month follow-up. J Rehabil Med. 2016;48(5):456-63. doi: 10.2340/16501977-2091. PubMed PMID: 27097785.

24. Luomajoki H, Kool J, de Bruin ED, Airaksinen O. Reliability of movement control tests in the lumbar spine. BMC Musculoskelet Disord. 2007;8:90. Epub 2007/09/14. doi: 1471-2474-8-90 [pii] 10.1186/1471-24748-90. PubMed PMID: 17850669; PubMed Central PMCID: PMC2164955.

25. Macedo LG, Saragiotto BT, Yamato TP, Costa LO, Menezes Costa LC, Ostelo RW, et al. Motor control exercise for acute non-specific low back pain. Cochrane Database Syst Rev. 2016;2:CD012085. doi: 10.1002/14651858.CD012085. PubMed PMID: 26863390.

26. O'Sullivan K, O'Sullivan P, Vibe Fersum K, Kent P. Better targeting care for individuals with low back pain: opportunities and obstacles. Br J Sports Med. 2017;51(6):489-90. doi: 10.1136/bjsports-2016-096612. PubMed PMID: 27697936.

27. Bauer CM, Heimgartner M, Rast FM, Ernst MJ, Oetiker S, Kool J. Reliability of lumbar movement dysfunction tests for chronic low back pain patients. Man Ther. 2016;24:81-4. doi: 10.1016/j.math.2016.02.013. PubMed PMID: 26980560.

28. Bauer CM, Rast FM, Ernst MJ, Oetiker S, Meichtry A, Kool J, et al. Pain intensity attenuates movement control of the lumbar spine in low back pain. J Electromyogr Kinesiol. 2015;25(6):919-27. doi: 10.1016/j.jelekin.2015.10.004. PubMed PMID: 26524940.

29. Bauer CM, Rast FM, Ernst MJ, Kool J, Oetiker S, Rissanen SM, et al. Concurrent validity and reliability of a novel wireless inertial measurement system to assess trunk movement. J Electromyogr Kinesiol. 2015;25(5):782-90. doi: 10.1016/j.jelekin.2015.06.001. PubMed PMID: 26126796.

30. Kent $P$, Laird R, Haines $T$. The effect of changing movement and posture using motion-sensor biofeedback, versus guidelines-based care, on the clinical outcomes of people with sub-acute or chronic low back pain- 
a multicentre, cluster-randomised, placebo-controlled, pilot trial. BMC Musculoskelet Disord. 2015;16:131. doi: 10.1186/s12891-015-0591-5. PubMed PMID: 26022102; PubMed Central PMCID: PMC4446825.

31. Lomond KV, Henry SM, Hitt JR, DeSarno MJ, Bunn JY. Altered postural responses persist following physical therapy of general versus specific trunk exercises in people with low back pain. Man Ther. 2014;19(5):42532. doi: 10.1016/j.math.2014.04.007. PubMed PMID: 24853255; PubMed Central PMCID: PMC4288452.

32. Lomond KV, Jacobs JV, Hitt JR, DeSarno MJ, Bunn JY, Henry SM. Effects of low back pain stabilization or movement system impairment treatments on voluntary postural adjustments: a randomized controlled trial. Spine J. 2015;15(4):596-606. doi: 10.1016/j.spinee.2014.10.020. PubMed PMID: 25452017; PubMed Central PMCID: PMC4375040.

33. Hodges PW, Tsao H, Sims K. Gain of postural responses increases in response to real and anticipated pain. Experimental brain research. 2015;233(9):2745-52. doi: 10.1007/s00221-015-4347-0. PubMed PMID: 26105752.

34. Stanton TR, Moseley GL, Wong AYL, Kawchuk GN. Feeling stiffness in the back: a protective perceptual inference in chronic back pain. Scientific reports. 2017;7(1):9681. doi: 10.1038/s41598-017-09429-1. PubMed PMID: 28851924; PubMed Central PMCID: PMC5575135.

35. Salamat S, Talebian S, Bagheri H, Maroufi N, Jafar Shaterzadeh M, Kalbasi G, et al. Effect of movement control and stabilization exercises in people with extension related non -specific low back pain- a pilot study. J Bodyw Mov Ther. 2017;21(4):860-5. doi: 10.1016/j.jbmt.2017.02.005. PubMed PMID: 29037640.

36. Ng L, Caneiro JP, Campbell A, Smith A, Burnett A, O'Sullivan P. Cognitive functional approach to manage low back pain in male adolescent rowers: a randomised controlled trial. $\mathrm{Br} J$ Sports Med. 2015;49(17):1125-31. doi: 10.1136/bjsports-2014-093984. PubMed PMID: 25618890.

37. O'Sullivan P. It's time for change with the management of non-specific chronic low back pain. Br J Sports Med 2012;46(4):224-7.

38. Tsao H, Druitt TR, Schollum TM, Hodges PW. Motor training of the lumbar paraspinal muscles induces immediate changes in motor coordination in patients with recurrent low back pain. J Pain. 2010;11(11):1120-8. doi: 10.1016/j.jpain.2010.02.004. PubMed PMID: 20434958.

39. Hodges PW, Tucker K. Moving differently in pain: a new theory to explain the adaptation to pain. Pain. 2011;152(3 Suppl):S90-8. doi: 10.1016/j.pain.2010.10.020. PubMed PMID: 21087823.

40. WHO. Adherence to long-term therapies: evidence for action. I.WHO Adherence to Long Term Therapies Project. II. Global Adherence Interdisciplinary Network. : WHO Library Cataloguing-in-Publication Data 20032003.

41. McLean SM, Burton M, Bradley L, Littlewood C. Interventions for enhancing adherence with physiotherapy: a systematic review. Man Ther. 2010;15(6):514-21. doi: 10.1016/j.math.2010.05.012. PubMed PMID: 20630793.

42. McLean S, Holden MA, Potia T, Gee M, Mallett R, Bhanbhro S, et al. Quality and acceptability of measures of exercise adherence in musculoskeletal settings: a systematic review. Rheumatology (Oxford). 2017;56(3):426-38. doi: 10.1093/rheumatology/kew422. PubMed PMID: 28013200.

43. Beinart NA, Goodchild CE, Weinman JA, Ayis S, Godfrey EL. Individual and intervention-related factors associated with adherence to home exercise in chronic low back pain: a systematic review. Spine J. 2013;13(12):1940-50. doi: 10.1016/j.spinee.2013.08.027. PubMed PMID: 24169445.

44. Hall AM, Kamper SJ, Hernon M, Hughes K, Kelly G, Lonsdale C, et al. Measurement tools for adherence to non-pharmacologic self-management treatment for chronic musculoskeletal conditions: a systematic review. Arch Phys Med Rehabil. 2015;96(3):552-62. doi: 10.1016/j.apmr.2014.07.405. PubMed PMID: 25108098.

45. Higgins JPT GSe. Cochrane Handbook for Systematic Reviews of Interventions Version 5.1.0 [updated March 2011]. The Cochrane Collaboration; 2011.

46. Costa Lda C, Maher CG, McAuley JH, Hancock MJ, Smeets RJ. Self-efficacy is more important than fear of movement in mediating the relationship between pain and disability in chronic low back pain. Eur J Pain. 2011;15(2):213-9. doi: 10.1016/j.ejpain.2010.06.014. PubMed PMID: 20655254. 
47. Di Pietro F, Catley MJ, McAuley JH, Parkitny L, Maher CG, Costa Lda C, et al. Rasch analysis supports the use of the Pain Self-Efficacy Questionnaire. Phys Ther. 2014;94(1):91-100. doi: 10.2522/ptj.20130217. PubMed PMID: 24009343.

48. Borsbo B, Gerdle B, Peolsson M. Impact of the interaction between self-efficacy, symptoms and catastrophising on disability, quality of life and health in with chronic pain patients. Disability and rehabilitation. 2010;32(17):1387-96. doi: 10.3109/09638280903419269. PubMed PMID: 20513205.

49. Bachmann C, Oesch, P., Bachmann, S. Recommendations for Improving Adherence to Home-Based Exercise. Phys Med Rehab Kuror. 2017; accepted fpr publication.

50. Moher D, Hopewell S, Schulz KF, Montori V, Gotzsche PC, Devereaux PJ, et al. CONSORT 2010 Explanation and Elaboration: updated guidelines for reporting parallel group randomised trials. Brit Med J. 2010;340:. doi: ARTN c869 DOI 10.1136/bmj.c869. PubMed PMID: ISI:000276157600007.

51. Higgins JPT LT, Chandler J, Tovey D, Churchill R. . Methodological Expectations of Cochrane Intervention Reviews. : Cochrane: London, ; 2016.

52. O'Cathain A, Thomas KJ, Drabble SJ, Rudolph A, Hewison J. What can qualitative research do for randomised controlled trials? A systematic mapping review. BMJ open. 2013;3(6). doi: 10.1136/bmjopen-2013002889. PubMed PMID: 23794542; PubMed Central PMCID: PMC3669723. 
Summary 
This dissertation addresses the assessment and treatment of the widespread complaint of non-specific low back pain. Active physiotherapy treatment of a defined subgroup, known as movement control impairment, was evaluated. Related topics that were reviewed are the clinimetric properties of one of the outcome measures and adherence to home exercise.

Chapter 1 provides background information on the current state of research concerning the impacts of low back pain on society and the individual patient. Furthermore, it describes active treatments using exercise, previous research regarding the search for subgroups within the non-specific low back pain patient group and the historical aspects of exercise methods.

The high prevalence rate of low back pain has been internationally documented. It is generally recognised that in the majority of cases complaints are temporary. However, there is a strong tendency towards recurrent episodes. The small number of chronic cases is responsible for immense costs in society. To find an effective method of preventing recurrences and chronification is very desirable.

Worldwide, clinical guidelines for the treatment of low back pain recommend therapy-based and home-based exercise. There is substantial evidence that exercise is effective in the prevention of low back pain. However, to date, no study has shown which exercise, or exercise combination, is most effective. The formation of subgroups and classifications within the non-specific low back pain group is widely recommended, with the aim of further tailoring effective treatments.

Movement control impairment is one subgroup of the non-specific low back pain group. Previous studies have established the clinical assessment and the classification of this subgroup. The most important clinical criteria are pain, combined with impaired control of the lumbar spine, both in static postures and during movement. These were assessed using a movement test battery. The treatment of this clinical presentation has shown most promising results in preliminary studies. However, there had been no study comparing an individually-targeted exercise program with a general, non-specific exercise program. The question of which exercises should be recommended for this subgroup is of great importance to clinical application.

Chapter 2 presents the protocol of our randomised controlled trial (RCT), in which two exercise programs for patients with subacute and chronic non-specific low back pain are compared (one exercise program specifically tailored for the movement control impairment, the other a non-specific general strengthening program). It contains detailed descriptions of the criteria and tests for the subgroup classification (illustrations are shown in the Appendix). Procedures, outcome measures and data analysis are described and conform to CONSORT criteria. Additionally, the rational for the research question is discussed. 
Chapter 3 reports the short-term results of the RCT, describing the methodological procedures, data analysis and outcomes pre- and post-treatment. The appendix (Chapter 3a) presents illustrations of the exercise programs. One hundred and six patients diagnosed with movement control impairment were included in the study and randomly assigned to one of two exercise programs. This multicentre trial was performed in 14 clinics and private practices in Switzerland. A total of 28 physiotherapists provided treatment. Additional personnel performed the measurements. Patients were instructed on the exercises in individual physiotherapy sessions and also received a home exercise program, which they were advised to follow for a period of one year. Both programs, the individuallytailored exercises to improve movement control impairment and the general strengthening program, reduced activity limitation and functional restriction significantly. We did not identify differences in outcomes between the two exercise programs in the short term. One explanation of this result could be a lack of contrast between the exercise programs. Initially the exercises between the groups differed distinctively. However, as soon as the patients in the experimental group had mastered the control exercises, they proceeded to exercises with higher demands. This led to an increasing convergence of the two exercise programs.

Chapter 4 presents the results of our RCT at the 6-month follow-up and one year after enrolment. Long-term follow-ups and data analysis are described.

The study shows that specifically-tailored exercises as a treatment for movement control impairment and non-specific general exercises in this patient group produced similar effects. Outcomes in both exercise groups showed significant improvements over one year. Therefore, both exercise programs can be recommended for patients with movement control impairment. Several explanations for these results are possible: firstly, the exercise programs were too similar, especially over the long term course of the treatment; secondly, other factors in the therapy, such as the therapeutic alliance between therapist and patient, may play a bigger role than the purely mechanical approach of exercise; and thirdly, multidimensional biopsychosocial aspects could have influenced the effects. We also showed that patients and therapists in both treatment groups performed with good adherence to the study protocol.

Chapter 5 reports a study in which we analysed the validity (construct validity, validity to change) of the patient-specific functional scale (PSFS), which was used as an outcome measure in the RCT. With data from our RCT, together with results from a similar study on patients with shoulder pain, the correlation between PSFS and well-established 'condition-specific' outcomes (Roland Morris Disability Questionnaire for back pain; Shoulder Pain and Disability Index for shoulder pain) was calculated. Our hypothesis of the expected correlation rate between the two outcomes at different time points was not confirmed.

We concluded that the application of PSFS as an outcome measure on a group level and in long term comparisons is not to be recommended. The well-established, condition- 
specific outcome measures are instead recommended. However, in every day clinical use the PSFS shows advantages and its results are very useful in monitoring short-term changes.

Chapter 6 presents the results of a qualitative study which was embedded into the quantitative design of the RCT. It evaluated the long-term adherence to home-based exercise. Forty-four participants were asked in written, open questions about their experiences regarding the exercise program over the course of one year. Participants pointed out several explanations for their good adherence to exercise: firstly, the role of improved selfconfidence through specific and individual instructions by the therapist; secondly, the perceived direct association between exercise and pain reduction; and thirdly, a lower restriction of daily live activities resulted in an increase in self-efficacy. Several participants mentioned the combination of the recommended exercises with a self-initiated training as a useful strategy to overcome barriers of "not enough time". We found no substantial differences between the two exercise programs. These insights into the perception of the participants are useful findings for future patient education information and for the support of self-efficacy in patients.

Chapter 7 discusses the results of this thesis in the context of recent evidence. Consideration is given to methodological reflections, theoretical implications, recommendations for clinical use and for future research.

The main results of this thesis endorse the importance of exercise in patients with low back pain and movement control impairment. Contrary to our expectations, we could not show any additional benefits of specific movement control exercises. Although current literature recommends subgrouping non-specific low back pain patients, no additional beneficial effect of specific treatment for this subgroup was detected.

We demonstrated that continuous functional improvement is supported by adherence to exercise. Participants highly valued their increase in confidence and self-efficacy and perceived these as supporting factors in their adherence to exercise.

In the clinical practise of exercise physiotherapy, the active training for long-term use in patients with $\mathrm{MCl}$ should be applied individually. Patients report that they benefit most from tailor-made treatment. Awareness of the association between pain and exercise is important. Coaching, aimed at combining therapeutic exercises with self-initiated training, supports adherence to exercise.

Assessment with the patient-specific functional scale is useful in a clinical context to document the short-term results of complaints. It cannot be recommended as a measurement at group level, or over the long term for research purposes.

Recommendations for future research include: methodological adjustments to the design of studies; incorporation of the influence of non-mechanical aspects in physiotherapy; and further studies into adherence to exercise. 


\section{Zusammenfassung}


Diese Doktorarbeit befasst sich mit der weitverbreiteten Krankheit von nichtspezifischen lumbalen Rückenschmerzen, ihrer Untersuchung und ihrer Behandlung. Für die beschriebene Subgruppe - Bewegungskontrolleinschränkung / movement control impairmentwird die aktive Physiotherapie evaluiert. Weitere Themen sind die angewendeten Testverfahren und die Adhärenz zu Heimübungsprogrammen.

Kapitel 1 vermittelt Informationen über die aktuellen Auswirkungen von lumbalen Rückenschmerzen auf die Gesellschaft und auf den Patienten. Des Weiteren werden die aktive Behandlung mittels Übungen, die Einführung von Subgruppen mit lumbalen Rückenschmerzen und die historischen Zusammenhänge von Übungsmethoden aufgezeigt.

Die hohe Rate des Auftretens von lumbalen Rückenschmerzen im Laufe des Lebens ist international gut dokumentiert. In den meisten Fällen sind diese Beschwerden vorübergehend. Es besteht aber eine deutliche Tendenz zu rezidivierenden Schmerzepisoden. Trotzdem verursachen die wenigen chronischen Verläufe der Krankheit immense Kosten für die Gesellschaft. Eine wirksame Prävention zur Vermeidung von Rezidiven und von Chronifizierung ist deshalb anzustreben.

Die Behandlung mit therapiebasierten und heimbasierten Übungsprogrammen bei Rückenschmerzen wird basierend auf einer hohen Evidenzrate weltweit von allen klinischen Leitlinien empfohlen. Übungsprogramme sind wirksam gegen Rückenschmerzen. Trotzdem konnte bisher noch keine Studie die optimal wirksame Übung oder Übungsabfolge aufzeigen. Die Bildung von Subgruppen und Klassifikationen des Krankheitsbildes wird empfohlen und soll eine wissenschaftliche Erforschung und eine effektive Behandlung ermöglichen.

Movement control impairment (Bewegungskontrolleinschränkung) ist eine Subgruppe von nichtspezifischen lumbalen Rückenschmerzen. Die klinische Untersuchung und anschliessende Klassifizierung wurde vorgängig erforscht. Wichtigste klinische Kriterien sind Schmerzen und mangelnde Kontrolle von lumbalen Wirbelsegmenten bei statischen Positionen und Bewegungen, welche mittels einer Bewegungstestbatterie erhoben werden. Die Behandlung dieser klinischen Präsentation erwies sich in Vorstudien als sehr vielversprechend. Es fehlte aber der Vergleich eines individuellen, testbasierten Übungsprogramms mit generellen unspezifischen Übungen. Die Frage, welche Übungen für diese Subgruppe zu empfehlen sind, entspricht einem grossen klinischen Bedarf. Zusätzlich könnten andere Faktoren, wie die Adhärenz zu Übungsprogrammen, die Resultate deutlich beeinflussen.

Kapitel 2 enthält das Protokoll der quantitativen, randomisierten, kontrollierten Studie (RCT engl: randomised controlled trial) zur Erforschung von zwei Übungsprogrammen für Patienten mit subakuten und chronischen lumbalen Rückenschmerzen (ein Übungsprogramm spezifisch gegen das movement control impairment und ein unspezifisches allgemeines Krafttraining). Detailliert werden die Kriterien und Tests zur Einteilung in die Subgruppe beschrieben und bildlich (siehe Anhang) dargestellt. Deutlich beschrieben sind 
der Ablauf der geplanten Studie, die geplanten Messverfahren und die Auswertungsmethodik. Erläutert wird die Argumentation zur Forschungsfrage.

Kapitel 3 präsentiert die kurzfristigen Forschungsresultate des RCT. Die detaillierten Abläufe der Studie, die Datenanalyse und die Resultate aller Messwerte vor und anschliessend an die physiotherapeutische Behandlung sind in diesem Artikel beschrieben. Im Anhang (Kapitel 3a) sind die vorgegebenen Übungen genau beschrieben. Einhundertsechs Patienten mit einer Bewegungskontrolleinschränkung wurden nach dem Zufallsprinzip in zwei Übungsgruppen eingeteilt. In 14 Kliniken und Privatpraxen wurden sie behandelt und erhielten ein Heimübungsprogramm, welches sie über ein weiteres Jahr anwenden sollten. Total 28 Physiotherapeuten erbrachten die Behandlungen und weitere Personen führten die Messungen durch. Sowohl die individuell angepassten Übungen zur Verbesserung der Bewegungskontrolle wie auch die generellen Kräftigungsübungen reduzierten die Aktivitäts- und Funktionseinschränkungen signifikant. Wir konnten kurzfristig keine Differenz der Verbesserungen zwischen den beiden Übungsprogrammen messen. Als ein Grund für dieses Resultat eruierten wir den fehlenden Kontrast zwischen den Übungsprogrammen. Zu Beginn des Trainings unterschieden sich die Übungen der zwei Gruppen sehr deutlich. Sobald aber die Patienten in der Experimentalgruppe die Kontrollübungen beherrschten, konnten sie auch zunehmende, belastende Übungen anwenden. Dies führte zu einer Angleichung der Übungsprotokolle.

Kapitel 4 präsentiert die Resultate des oben vorgestellten RCT mit nachfolgenden Messungen nach 6 Monaten und einem Jahr. Detailliert beschrieben sind der langfristige Verlauf der Studie und die verwendeten Datenanalysen.

Die Studie zeigt, dass die spezifischen Übungen zur Behandlung von Movement Control Impairment und die unspezifischen generellen Übungen in dieser Patientengruppe gleich effektiv sind. Nochmals haben sich die Resultate in beiden Übungsgruppen über den ganzen Zeitverlauf signifikant verbessert. Für Patienten mit einem Movement Control Impairment können beide Übungsprotokolle empfohlen werden. Verschiedene Erklärung für diese Resultate sind denkbar: Erstens, die Übungsprogramme sind zu ähnlich, vor allem im Langzeitverlauf der Behandlung; zweitens, andere Faktoren der Therapie wie die therapeutische Allianz zwischen Physiotherapeut und Patienten könnten eine grössere Rolle spielen, als die rein mechanischen Einflüsse eines Übungsprogrammes; und drittens, könnten multidimensionale bio-psycho-soziale Aspekte der Behandlung den Effekt der Behandlung relevant beeinflusst haben. Abschliessend können wir sagen, dass sowohl Patienten wie Therapeuten in beiden Übungsgruppen eine gute Adhärenz zum Studienprotokoll aufwiesen.

Kapitel 5 beinhaltet eine Studie, welche die Validität (construct validity, validity to change) des in der Studie verwendeten Messverfahrens „patient specific functional score“ (PSFS) analysiert. Mit Daten aus dem oben beschriebenen RCT an Patienten mit lumbalen Rückenschmerzen und den Resultaten einer entsprechenden Studie an Patienten mit 
Schulterschmerzen wurden die Korrelationen zwischen PSFS und bereits evaluierten „condition-specific" Messresultaten (Roland-Morris Disability Score für Rückenschmerzen; Shoulder Pain and Disability Index für Schulterschmerzen) gemessen. Unsere Hypothese einer erwarteten Korrelationsrate zwischen den Messverfahren zu verschiedenen Zeitpunkten konnte nicht bestätigt werden.

Wir konnten daraus schliessen, dass der PSFS für Messungen auf Gruppenstufe und im Langzeitvergleich nicht mehr zu empfehlen ist. Vorzugsweise sollten die etablierten Messungen verwendet werden. Im klinischen Alltag bringt der PSFS Vorteile und die Resultate sind als Verlaufskontrolle kurzfristig sehr gut anwendbar.

Kapitel 6 präsentiert die Resultate einer qualitativen, in das quantitative Design eingebetteten Studie zur Langzeitadhärenz in den Heimübungsprogrammen. Vierundvierzig Teilnehmer des RCT wurden schriftlich zu ihren Erkenntnissen während der Übungsprogrammdauer von einem Jahr befragt. Die Teilnehmer der Studie haben mehrere Gründe für die gute Adhärenz zu den Übungen hervorgehoben: Erstens, den Wert der gestärkten Selbstsicherheit durch genaue individuelle Instruktion durch den Therapeuten; zweitens, den verspürten direkten Zusammenhang zwischen Übung und Nachlassen des Schmerzes; und drittens, weniger Einschränkungen im täglichen Leben wegen erhöhter Selbsteffizienz. Als eine wertvolle Strategie zur Überwindung von Hinderungsgründen wie „zu wenig Zeit" erwähnten mehrere Teilnehmer das Einbinden der Rückenübungen in ein Training der eigenen Wahl. Zwischen den beiden Übungsgruppen gab es keine deutlichen Unterschiede über angegebene Themen und Strategien. Diese Einsichten in das Erleben der Studienteilnehmer sind wertvolle Erkenntnisse für zukünftige Patienteninformationen und für die Unterstützung der Selbsteffizienz der Patienten.

Kapitel 7 präsentiert die wichtigsten Resultate und diskutiert diese im Kontext zur neuesten Evidenzlage. Ebenfalls werden methodologische Reflexionen, theoretische Implikationen, Empfehlungen für die klinische Praxis und für weitere Forschungsthemen abgegeben.

Die Hauptresultate dieser Thesis unterstreichen die Wichtigkeit von Übungsprogrammen bei Patienten mit lumbalen Rückenproblemen und movement control impairment. Anders als erwartet konnten wir den Zusatznutzen von spezifischen movement controlÜbungen nicht nachweisen. Obwohl die aktuelle Literatur die Anforderung von Subgruppierungen von nichtspezifischen Rückenschmerzen immer wieder betont, können wir mit diesem Verfahren, mit dieser Subgruppe, keinen Unterschied zwischen den Übungsprogrammen feststellen.

Wir konnten zeigen, dass anhaltende Effekte durch Übungsadhärenz unterstützt werden. Die Teilnehmer der Studie erachteten die zunehmende Selbstsicherheit und die Selbsteffizienz als wichtigen Faktor zur Adhärenz zu den Übungsprogrammen.

Im klinischen Alltag der Physiotherapie sollten aktive Langzeitübungen bei Patienten mit $\mathrm{MCl}$ individuell angepasst werden. Die Patienten möchten genaue Instruktionen. Das Erkennen des Zusammenspiels von Schmerz und Übungen ist wichtig. Eine Verbindung 
der Übungen mit einer selbstinitiierten regelmässigen Aktivität erleichtert die Adhärenz zu den Übungen.

Die Untersuchung mittels dem patient specific functional scale (PSFS) ist sehr gut geeignet, um den kurzfristigen Verlauf der Beschwerden zu dokumentieren. Als Gruppenvergleich oder als Langzeitverlaufsmessung würden wir in Zukunft davon absehen.

Die Forschungsempfehlungen beinhalten Themen wie: Methodologische Anpassungen im Design der Studie, den Einfluss von nicht-mechanischen Aspekten in der Physiotherapie und weitere Entwicklungen im Bereich der Adhärenz von Übungen. 

Valorisation 
This thesis provides us with additional knowledge on exercise interventions for patients with subacute and chronic low back pain. Earlier chapters addressed the scientific and clinical aspects of both our quantitative and qualitative investigations. This valorisation section will discuss further insights gained from the thesis, such as innovation, the social and economic relevance of the findings, proposed potential target group and description of the issues associated with knowledge transfer.

\section{INNOVATION}

Exercises for the treatment of low back pain (LBP) are widely prescribed in clinical practice and are recommended in many guidelines (1-3). These guidelines refer to the high quality evidence on exercise therapy. However, it is also noted that the particular types of exercise which are most effective are still largely unknown.

The data outlined in this thesis provide clinicians with the relevant arguments to make an evidence-based clinical decision $(4,5)$. When deciding on exercise therapy for patients with LBP, physiotherapists base their choice of exercises on various assessments and clinical decisions. The decision on which type of exercise intervention to apply is based both on the physical presentation of the patient and on the psychological and social aspects of the patient's situation. This has led to heterogeneous exercises for the treatment of low back pain, with effects on: firstly, the evaluation of the individual treatment; secondly, the comparison between treatments; and lastly, the scientific analysis of treatment outcomes. The results of this thesis have defined standardised exercise treatments and analysed outcomes for a new subgroup of patients with LBP and movement control impairment $(6,7)$.

The main focus of this thesis is on the long-term effects of exercise, since it is known that the chronic progression of low back pain is responsible for major social and personal problems (8). We expected to gain new insights through focusing on a specific subgroup of patients with $\operatorname{LBP}(9,10)$. In this randomised, controlled, multicentre study, the selected subgroup of patients with low back pain and movement control impairment (MCl) presented with a measurable mechanical movement control problem (11-13). We hypothesised that this subgroup would respond more favourably to a specific tailored exercise program compared to a general non-specific programme. Our results revealed, however, that this subgroup benefitted equally from both types of exercise programme. Patients improved in function and activity restriction following both types of exercise programme, as long as there was adherence to the programme. The improvement was maintained over the course of one year for both programmes. Thus, both exercise programs can be recommended for this patient group, with the expectation that they bring improved function and less pain in the short and long terms. It seems that adherence is more important than the specificity of the exercise programme.

The duration of physiotherapy treatment and exercise instruction to prevent or improve LBP is usually restricted. Following intervention, patients are usually recommended 
to adhere to their exercise programme over the long term. We know from previous studies that adherence to an exercise program decreases substantially over the long term (14, 15). In the main study of this thesis, the adherence rate to exercise, of at least twice a week for both exercise programmes, was high at $60 \%$ after 6 months and $48 \%$ after one year. The results of the qualitative evaluation of this one-year, self-treatment phase indicated that patients felt reassured that they were exercising correctly and, as a result, chose to include the recommended programme into their regular, self-initiated physical activity programme. For clinicians, these results provide important insights into improving exercise adherence when advising patients on specific exercise strategies. Strategies for long-term exercise adherence help patients to commit over the long term, support their self-management capabilities and adapt to individual demands (16).

\section{SOCIAL AND ECONOMIC RELEVANCE}

Low back pain is ranked as the greatest contributor to global disability, measured in terms of Years lived with Disability (YLD) (17). A single episode of back pain usually runs its course with a favourable outcome in the majority of patients and has no significant social or economic impact. However, in those populations with subacute and chronic low back pain, work absenteeism or long-term disability are drivers of unfavourable social and economic costs (18). The need for cost-effective and evidence-based treatments for these patient groups, in order to restore their function and allow their renewed contribution to the work force, is clear.

In addition to confirming previously published results on the beneficial effects of exercise, this thesis also provides evidence of the continuing favourable effects over the course of one year $(1,2)$. Regular exercise is particularly effective in patients with subacute and chronic low back pain and movement control impairment. We recommended that physiotherapists guide patients through the learning process with patient-centred instruction until the patient has developed sufficient confidence in the exercise routine and its outcomes (16).

The long-term improvement in health, as described above, could have positive effects on both the social and economic costs. However, this thesis did not assess the costs of treatments against their impact on the economic burden. Neither was a social cost-benefit analysis of alternative therapeutic options for patients with low back pain performed.

\section{TARGET GROUP}

The studies in this thesis are of relevance to the large group of patients who suffer from longer-term low back pain and, in particular, to patients who present with signs of movement control impairment. This thesis has shown that adherence to a regular exercise programme can measurably improve function and reduce activity restriction due to pain.

The results also aid medical decision-makers who want to prescribe evidence-based treatments to their patients. In the context of physiotherapy treatment, the instruction, 
motivation and coaching of patients to perform exercise, as recommended in the various studies of this thesis, is a safe and highly accepted treatment option.

\section{TRANSFER OF KNOWLEDGE}

The transfer of new knowledge into clinical practice has been described as difficult, particularly in the context that experimental study designs are not seen as reflective of the everyday needs of clinics $(19,20)$. Our multicentre study demanded high acceptance from the involved professionals. Fourteen clinics and over fifty physiotherapists provided assessment, treatment and control support during the treatment phase of our project. The implementation of the two treatment arms faced no major difficulties, since both were already widely used in clinical practice. It became clear that the provided exercises, at least from the viewpoint of our participants, formed a feasible and adequate treatment option.

Further results described in this thesis lead to recommendations to clinicians on motivational and coaching strategies to enable patients to stay adherent to exercise over the long term (16). The provision of knowledge on the positive prospects for patients who present with signs and symptoms of movement control impairment, if they follow either of the two treatments described in this thesis, should be an essential part of physiotherapy treatment.

\section{Conclusion}

Exercise therapy is a well-accepted treatment option by clinicians and patients suffering from low back pain. Based on the results of our large, randomised, controlled, multicentre trial, we were not able to find a final answer to the long sought-after question, "Which exercise is best for whom?".

We have shown that patients with LBP and movement control impairment benefit from two types of exercise programme. Both programmes can clearly be recommended for treatment of this patient group. This improvement not only increases functional ability, but may also have favourable impacts on their social and economic burdens.

For future studies, an analysis of the costs and benefits of alternative therapeutic exercise treatments, which include clear recommendations and coaching on exercise adherence, and their assumed impacts on the social and economic burdens may be of interest. 


\section{References}

1. Bundesärztekammer (BÄK) KBK, Arbeitsgemeinschaft der Wissenschaftlichen Medizinischen Fachgesellschaften (AWMF). Nationale Versorgungsleitlinie Nicht-spezifischer Kreuzschmerz - Langfassung. 2017. Available from: www.kreuzschmerz.versorgungsleitlinien.de.

2. Koes BW, van Tulder M, Lin CW, Macedo LG, McAuley J, Maher C. An updated overview of clinical guidelines for the management of non-specific low back pain in primary care. Eur Spine J. 2010;19(12):207594.

3. Macedo LG, Bostick GP, Maher CG. Exercise for prevention of recurrences of nonspecific low back pain. Phys Ther. 2013;93(12):1587-91.

4. Childs JD, Flynn TW. Clinical decision making for low back pain: a step in the right direction. J Orthop Sports Phys Ther. 2014;44(1):1-2.

5. Rabin A, Shashua A, Pizem K, Dickstein R, Dar G. A clinical prediction rule to identify patients with low back pain who are likely to experience short-term success following lumbar stabilization exercises: a randomized controlled validation study. J Orthop Sports Phys Ther. 2014;44(1):6-B13.

6. Brennan GP, Fritz JM, Hunter SJ, Thackeray A, Delitto A, Erhard RE. Identifying subgroups of patients with acute/subacute "nonspecific" low back pain: results of a randomized clinical trial. Spine (Phila Pa 1976). 2006;31(6):623-31.

7. Kent $P$, Keating JL, Leboeuf-Yde C. Research methods for subgrouping low back pain. BMC Med Res Methodol. 2010;10:62.

8. Hartvigsen J, Hancock MJ, Kongsted A, Louw Q, Ferreira ML, Genevay S, et al. What low back pain is and why we need to pay attention. Lancet. 2018.

9. Dankaerts W, O'Sullivan P, Burnett A, Straker L, Davey P, Gupta R. Discriminating healthy controls and two clinical subgroups of nonspecific chronic low back pain patients using trunk muscle activation and lumbosacral kinematics of postures and movements: a statistical classification model. Spine (Phila Pa 1976). 2009;34(15):1610-8.

10. Karayannis NV, Jull GA, Hodges PW. Physiotherapy movement based classification approaches to low back pain: comparison of subgroups through review and developer/expert survey. BMC Musculoskelet Disord. 2012;13:24.

11. Saner J, Kool J, de Bie RA, Sieben JM, Luomajoki H. Movement control exercise versus general exercise to reduce disability in patients with low back pain and movement control impairment. A randomised controlled trial. BMC Musculoskelet Disord. 2011;12:207.

12. Saner J, Kool J, Sieben JM, Luomajoki H, Bastiaenen $\mathrm{CH}$, de Bie RA. A tailored exercise program versus general exercise for a subgroup of patients with low back pain and movement control impairment: A randomised controlled trial with one-year follow-up. Man Ther. 2015;20(5):672-9.

13. Saner J, Sieben JM, Kool J, Luomajoki H, Bastiaenen $\mathrm{CH}$, de Bie RA. A tailored exercise program versus general exercise for a subgroup of patients with low back pain and movement control impairment: Shortterm results of a randomised controlled trial. J Bodyw Mov Ther. 2016;20(1):189-202.

14. Friedrich M, Gittler G, Arendasy M, Friedrich KM. Long-term effect of a combined exercise and motivational program on the level of disability of patients with chronic low back pain. Spine (Phila Pa 1976). 2005;30(9):995-1000.

15. Beinart NA, Goodchild CE, Weinman JA, Ayis S, Godfrey EL. Individual and intervention-related factors associated with adherence to home exercise in chronic low back pain: a systematic review. Spine J. 2013;13(12):1940-50.

16. Saner Jeannette, Bergman Esther M, de Bie Rob A., Sieben Judith M.,. Patients' strategies and barriers to successful long-term adherence to home-based exercise programmes in physiotherapy. . submitted. 2018.

17. Hoy D, March L, Brooks P, Blyth F, Woolf A, Bain C, et al. The global burden of low back pain: estimates from the Global Burden of Disease 2010 study. Ann Rheum Dis. 2014;73(6):968-74. 
18. Juniper M, Le TK, Mladsi D. The epidemiology, economic burden, and pharmacological treatment of chronic low back pain in France, Germany, Italy, Spain and the UK: a literature-based review. Expert opinion on pharmacotherapy. 2009;10(16):2581-92.

19. Scurlock-Evans L, Upton P, Upton D. Evidence-based practice in physiotherapy: a systematic review of barriers, enablers and interventions. Physiotherapy. 2014;100(3):208-19.

20. Kerry R. Expanding our perspectives on research in musculoskeletal science and practice. Musculoskeletal science \& practice. 2017;32:114-9. 
Acknowledgment 
When I was given the opportunity to do a PhD, I talked, discussed and outweighed advantages and disadvantages of the project with my nearest friends and family, my colleagues at the university and in my business. All of them ensured me to help me and support my research career. This promise lasted through the past years and I will be for ever thankful to them.

First and most important my sincere thanks go to my husband Christoph and my children Fabienne and Joel. Without their constant encouragement, their support in domestic tasks, up to running the administration of my private practice and first proofreading of my manuscripts, this thesis would not be completed. I thank you deeply for the inspirational discussions around the topic at the family table and the patience and understanding you showed towards me in times of stress and doubt.

I and with me this thesis had the support of several "doctor fathers" and "doctor mothers" who led me through the process and to whom I owe all I learned during this project. When Prof. Dr. Hannu Luomajoki, who had the idea for and planned the main trial and Dr. Jan Kool, former director of research and development, at the Zurich University of Applied Sciences, approached me with the request to manage the study, I agreed enthusiastically. What followed was a very intense collaboration and steep learning curve for all of us, as this trial was one of the first bigger projects at the recently founded Research Department of the Institute of Physiotherapy in Winterthur. Thank you very much for giving me the opportunity, many thanks for your never ending support, your expert advice and the valuable discussions.

My special thanks go to Prof. Dr. Rob A. de Bie, and Dr. Judith M. Sieben, my promotors from the Department of Epidemiology, School of Public Health and Primary Care (CAPHRI), who guided me through the PhD process with great overview. Judith, I thank you very much for your constructive and always prompt feedback. I have learned so much by following your challenging questions and truly appreciated our "sparring partner" rounds. Many thanks go to Rob, who encouraged me over the years and never lost faith in my abilities. You challenged me in the right moments and stepped in with advice if needed.

I would like to express my gratitude to my co-authors who participated to a great extent in the publications for this thesis. Dr. Carolien H.G. Bastiaenen, Prof. Dr. Thilo O. Kromer whom I met at the Maastricht PhD meetings in Switzerland, where we shared and developed ideas. Thank you sincerely. Many thanks go to Dr. Esther M. Bergman, who introduced me to qualitative research methods and generously shared her experiences with me.

A thesis project could never be undertaken without the support of colleagues and staff at work. I would like to thank my co-lecturers of the musculoskeletal physiotherapy team at the ZHAW, Monika Bodmer, Agnes Verbay, Karin Lutz, Stefan Jan, Winfried Schmidt and Arjen van Duijn. You took over many of my tasks and forgave me if I had my thoughts somewhere else. Thanks go to my colleagues of the Physiotherapy research and development team at the ZHAW, especially to Prof. Dr. Markus Wirz, they always had an 
open ear and helped in finding solutions. Research assistant Christa Wachter, our 24 hours "guardian" of the enrolment telephone deserves a special thank you. In the recruitment phase for our project you played an important role.

The backing and the support of the Zurich University of Applied Sciences, Department of Health, Institute of Physiotherapy especially by the Director Prof. Dr. Astrid Schämann and Prof. Dr. Cécile Ledergerber, Head of BSc in Physiotherapy Programme, André Meichtry for statistics advice, Karen Linwood for proof reading, Yolanda Mohr-Häller for viewing videos and many others deserve a special thank you.

Many thanks go to my colleagues in my practice, Claudia Lochau PT and Carina Schön PT, who were willing to take up more and more responsibilities and patient appointments during the hectic phase of the project.

Fourteen clinics and private practices were involved in the main trial of this thesis. Over 50 physiotherapists were involved in recruitment, assessment and treatment of patients. Their contribution and enthusiasm for research was vital. Sincere thanks are given to them all.

Many friends showed me their support and sympathy during this PhD project. Your interest in my research and your encouragement are of the highest value. Thank you very much indeed. A special thank you goes to Dr. Ursula Gartenmann-Pauls, who not only read and corrected my thesis, she also commented with a very valuable outside view.

I have probably forgotten to thank numerous colleagues and friends who supported me or whom I bored with my research projects and still showed me their sympathy. Sincere thanks are given to them all. 

About the autor 


\section{Curriculum vitae}

Jeannette Saner-Bissig was born in 1957 in Switzerland. She completed her physiotherapy training at the University Hospital in Zurich in 1981. In 1986 she gained advanced diplomas in manual and manipulative physiotherapy from the Auckland University of Technology (AUT), Auckland, New Zealand and the New Zealand Manipulative Therapists Association (OMT). She worked as physiotherapist in various private practices around Zurich and in her own practice from 1987 to 2015.

Alongside the clinical work she worked as a lecturer for Anatomy, Manual Therapy and Automobilisation LWS in physiotherapy schools in Switzerland. She was co-founder of the McKenzie Institute Switzerland, Germany and Austria, she has a Diploma in Mechanical Diagnosis and Therapy and was Instructor of the McKenzie Institute International until 2005. In 2004 she received a Master of Science in Science at The Open University, Milton Keynes, UK.

Jeannette is currently a lecturer in the physiotherapy Bachelor Degree programme at the Institute of Physiotherapy, School of Health Professions at the Zurich University of Applied Sciences based in Winterthur. 


\section{Memberships}

Physioswiss

The McKenzie Institute

FH Schweiz
Swiss Physiotherapy Association

Mechanical Diagnosis and Therapy

Nationaler Fachhochschulen-Absolventenverband

\section{Publications}

Saner J, Kool J, Sieben JM, Luomajoki H. (2011). Movement control exercises versus general exercise to reduce disability in patients with low back pain and movement control impairment. A randomized controlled trial. BMC Musculoskel Dis;12:207.

Luomajoki, Hannu; Saner, Jeannette (2012). Bewegungskontrolldysfunktion als Subgruppe von unspezifischen Rückenschmerzen. Manuelle Medizin, 50, 387-392. Peer reviewed.

Saner, Jeannette (2014). Case Study „Karin“: wie behandeln wir das movement control impairment? McKenzie Newsletter, Jan

Saner J, Sieben JM, Kool J, Luomajoki H, Bastiaenen CHG, De Bie RA. (2015) A tailored exercise program versus general exercise for a subgroup of patients with low back poain and movement control impairment: A randomized controlled trial with one-year follow up. Manual Therapy; 20:672-9.

Saner, Jeannette; Luomajoki, Hannu; Sieben, Judith M; Kool, Jan; Bastiaenen, Carolien H.G.; de Bie, Rob A. (2015). Movement control exercise versus general exercise to reduce disability in patients with low back pain: randomized controlled multicentre study. In: Physiotherapy . WCPT Singapur 2015. (101:e917-e918 · May). Singapur: Elsevier.

Saner J, Sieben JM, Kool J, Luomajoki H, Bastiaenen CH, de Bie RA. (2016). A tailored exercise program versus general exercise for a subgroup of patients with low back pain and movement control impairment: Short-term results of a randomised controlled trial. J Bodyw Mov Ther.;20(1):189-202.

Kromer Thilo O. SJ, Sieben Judith M. , Bastiaenen Caroline H.G. (2018). The Patientspecific Functional Scale - its constuct validity and validity to change in patients with shoulder and low back pain. submitted.

Saner Jeannette, Bergman Esther M., de Bie Rob A., Sieben Judith M., (2018). Patients' strategies and barriers to successful long-term adherence to home-based exercise programmes in physiotherapy. submitted. 


\section{Books}

Saner-Bissig, Jeannette (2007). Hrsg. „McKenzie- Mechanische Diagnose und Therapie“, Verlag Georg Thieme

Robin.A McKenzie (1987) "Die lumbale Wirbelsäule", Spinal Publications, Waekanae, Deutsche Übersetzung

\section{Conference Contributions}

Saner, Jeannette; Kool, Jan; Luomajoki, Hannu; de Bie, Rob A; Sieben, Judith M (2012). Movement control exercise versus general exercise to reduce disability in patients with low back pain and movement control impairment. FysioCongres Maastricht 2012 (6. November 2012). Maastricht NL: KNGF. Oral presentation

Saner, Jeannette; Kool, Jan; Luomajoki, Hannu, de Bie, Rob A; Sieben, Judith M (2012). Movement control exercise versus general exercise - patient and therapist treatment adherence. FysioCongress Maastricht 2012 (7. November 2012). Maastricht NL. KNGF. Oral presentation

Saner, Jeannette; Kool, Jan; Luomajoki, Hannu; Sieben, Judith M; de Bie, Rob A; (2013). Movement control exercise versus general exercise - patient and therapist adherence to exercise and study protocol. Symposium Muskuloskelettale Physiotherapie - Messungen was bringts. (2. März 2013). Winterthur, CH. Oral presentation

Saner, Jeannette; Sieben, Judith .M.; Kool, Jan; Luomajoki, Hannu; Bastiaenen, Carolien.H.G.; De Bie, Rob A. (2013). Which types of exercises are beneficial for which patient with low back pain?. Movement control versus general exercise - a RCT. Pain in Europe. 8th Biennial EFIC Congress. Florence, I, 2013: EFIC. Poster presentation

Saner, Jeannette; Luomajoki, Hannu; Sieben, Judith M, Kool, Jan; Bastiaenen, Carolien H.G.; de Bie, Rob A.. (2015). Movement control exercise versus general exercise to reduce disability in patients with low back pain: randomized controlled multicentre study. WCPT Singapur 2015. Physiotherapy (101:e917-e918 May 2015). Oral presentation 
